\title{
Digital Circuit Wear-out due to Electromigration in Semiconductor Metal Lines
}

\author{
A Thesis Presented to the \\ Electrical Engineering Department Faculty of \\ California Polytechnic State University, San Luis Obispo \\ In Partial Fulfillment \\ Of the Requirements for the \\ Master of Science Degree in Electrical Engineering
}

By

Gregory Ross Wilkinson

November 2009 
(C) 2009

Gregory Ross Wilkinson

ALL RIGHTS RESERVED 


\section{COMMITTEE MEMBERSHIP}

TITLE: Digital Circuit Wear-out due to Electromigration in Semiconductor Metal Lines

AUTHOR: $\quad$ Gregory Ross Wilkinson

DATE SUBMITTED: $\quad$ November 2009

COMMITTEE CHAIR: Dr. John Oliver, Assistant Professor Electrical Engineering

COMMITTEE MEMBER: Dr. James Harris, Professor Emeritus Electrical Engineering

COMMITTEE MEMBER: Dr. Alberto Jimenez, Professor Mathematics 


\section{ABSTRACT \\ Title: Digital Circuit Wear-out due to Electromigration in Semiconductor Metal Lines Author: Gregory Ross Wilkinson}

With the constant scaling of semiconductor devices, reliability of these devices is a huge concern. One of the biggest reliability issues is a phenomenon known as electromigration (EM) [1] [2]. Electromigration is the transport of material caused by the gradual movement of the ions in a conductor due to the momentum transfer between conducting electrons and diffusing metal atoms [27]. The damage induced by electromigration appears as the formation of voids and hillocks, resulting in electrical discontinuity.

Based on previous Electromigration research [15], I have created a tool chain that identifies where electromigration is likely to occur in large-scale integrated circuits. Using this tool chain, it is possible to identify the mean-time to failure (MTTF) of several common and high priority circuits such as complex adders and memories. Furthermore, this tool chain allows designers to isolate weak-points in these circuits to improve the overall MTTF of the circuit. The result is that with a few simple changes, circuits can be redesigned to increase the MTTF, at minimal cost to the system. 


\section{ACKNOWLEDGEMENTS}

First of all, I would like to express my gratitude towards Dr. John Oliver for giving me the privilege to work on this research project. I fully appreciate the time, effort, and guidance he provided me.

I would like to give special thanks to Emanuel Tarog for being a great partner on this project and being so patient along the way. His knowledge and constant advice have helped me in completing such a great project.

I would like to thank Dr. James Harris for being an excellent committee member and constantly motivating me towards success.

I would like to thank Dr. Alberto Jimenez for giving me great advice on my educational and career goals throughout the past five years.

Finally, I would like to thank my family and friends for their love and support throughout my educational career. 


\section{TABLE OF CONTENTS}

LIST OF FIGURES

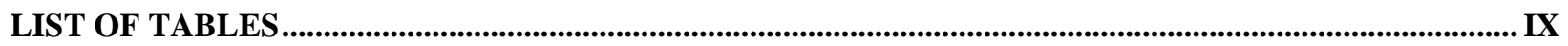

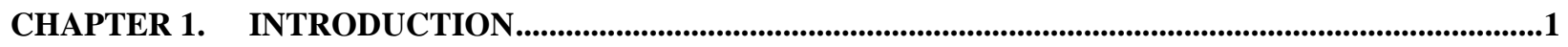

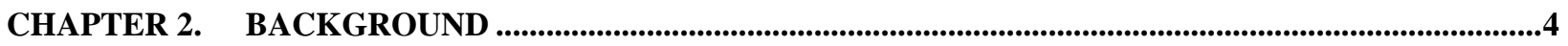

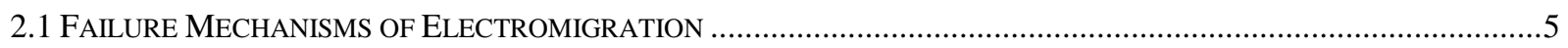

2.1.1 Metallurgical Statistical Properties of the Conducting Film ..................................................................

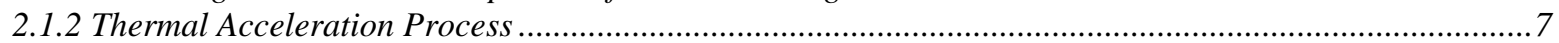

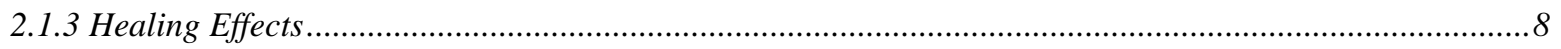

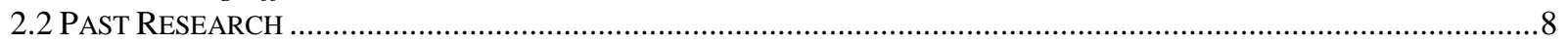

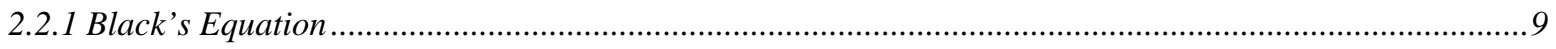

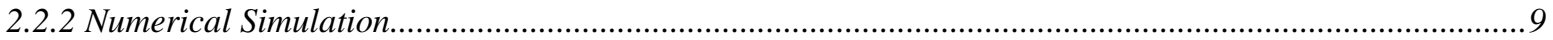

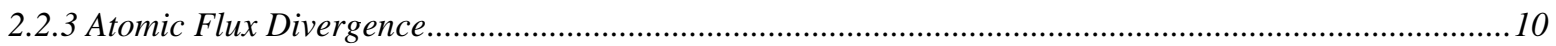

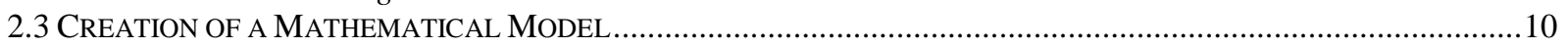

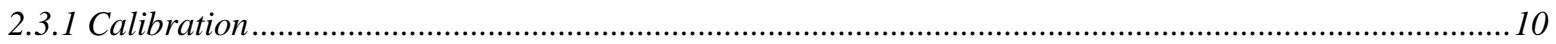

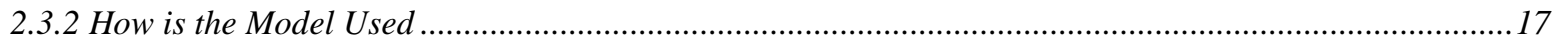

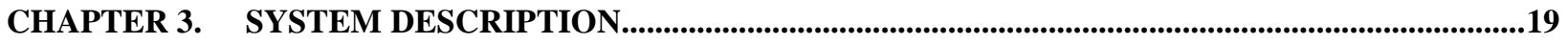

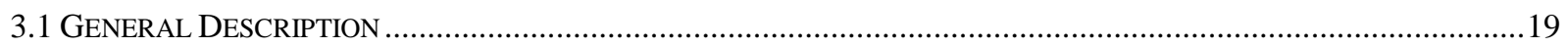

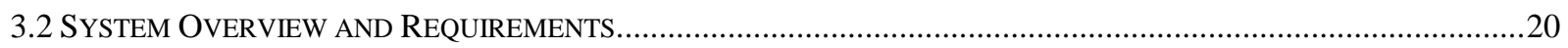

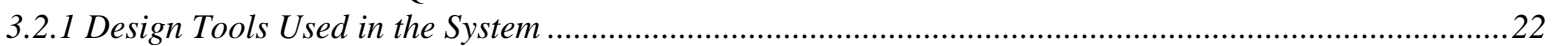

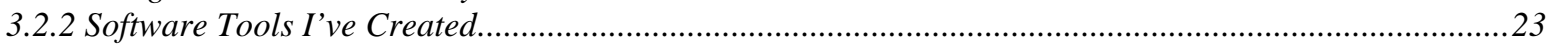

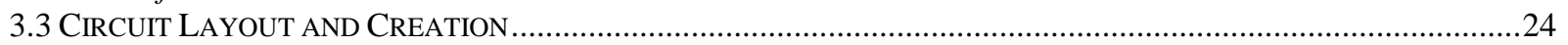

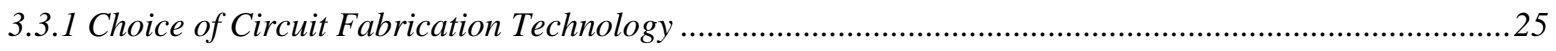

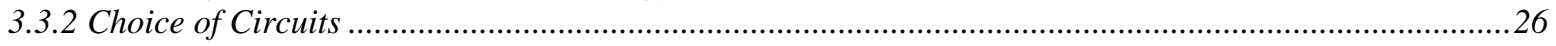

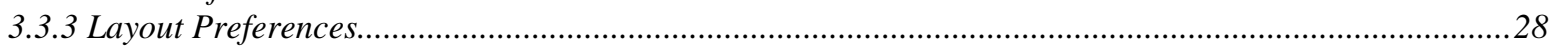

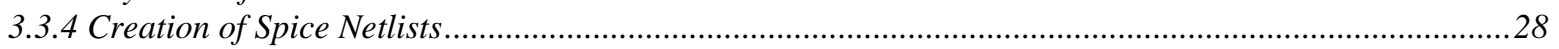

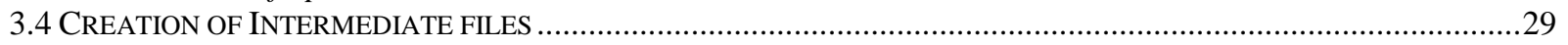

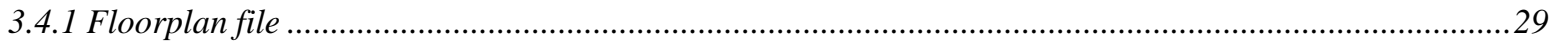

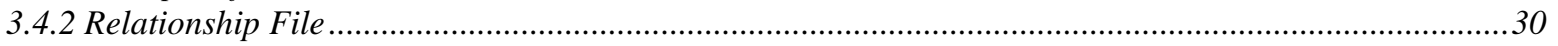

3.4.3 Manual creation of floorplans and relationship files.......................................................................

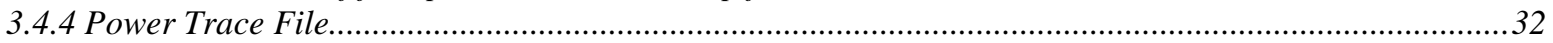

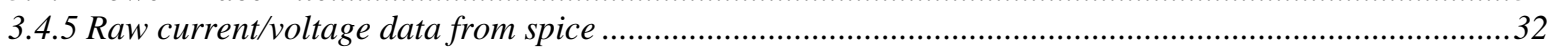

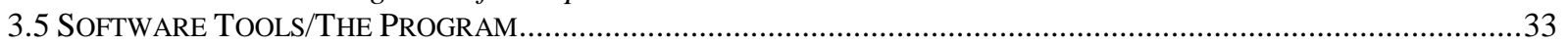

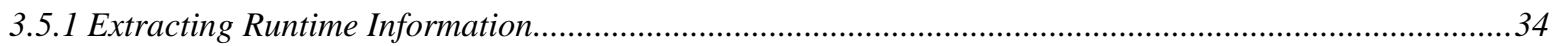

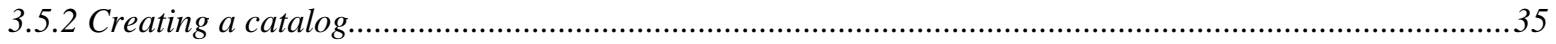

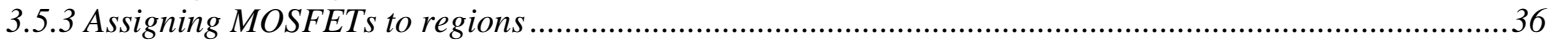

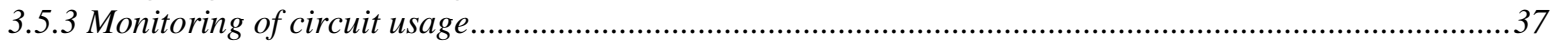

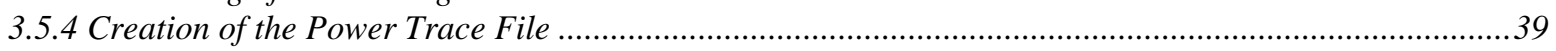

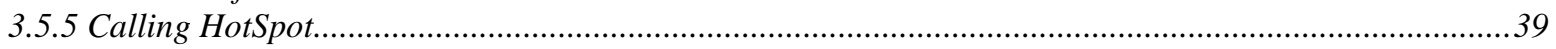

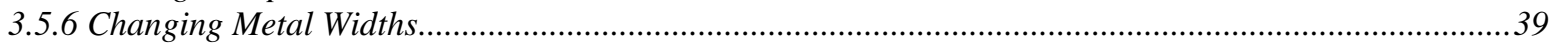

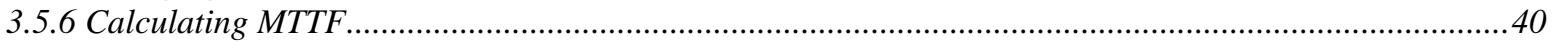

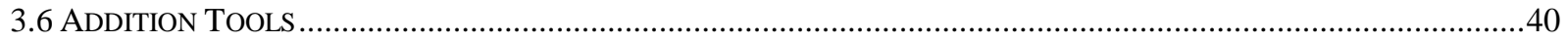

3.6.3 Converting the LTSpice Utility data file ......................................................................................... 41

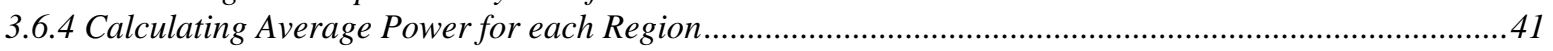

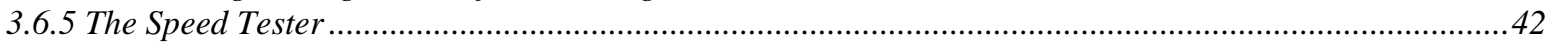

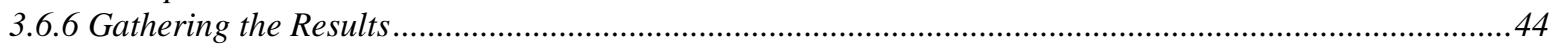

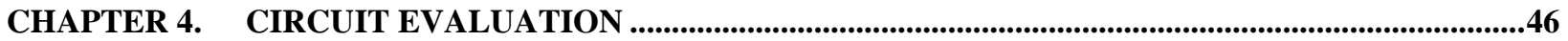

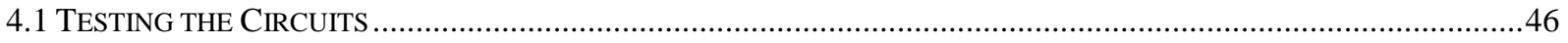

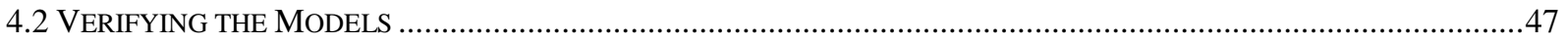




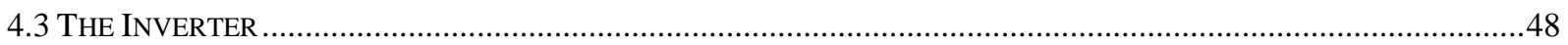

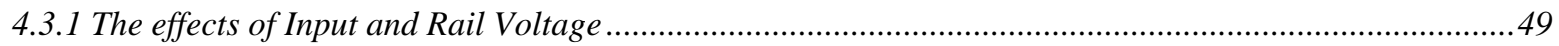

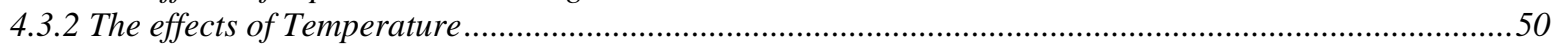

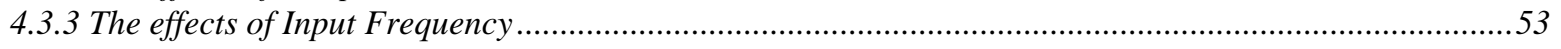

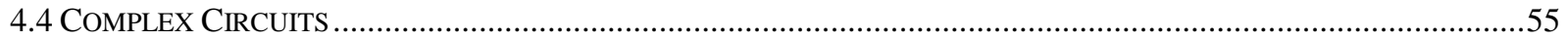

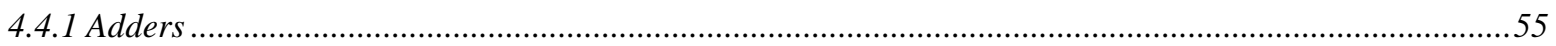

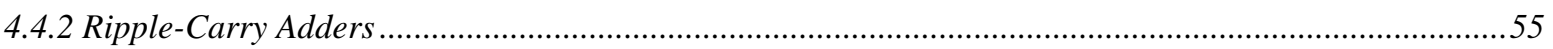

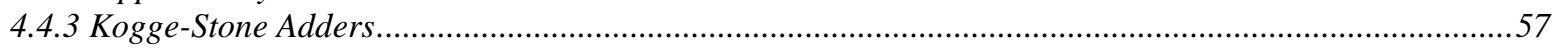

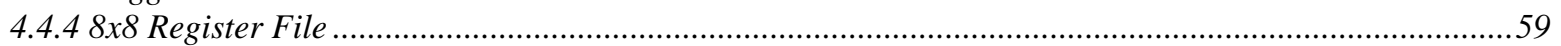

4.5 FAILURES, ASSOCIATED PERFORMANCE FLAWS AND POTENTIAL FIXES ......................................................61

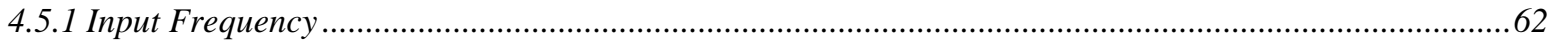

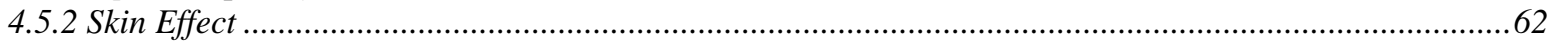

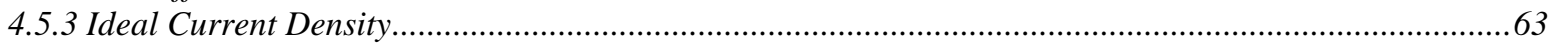

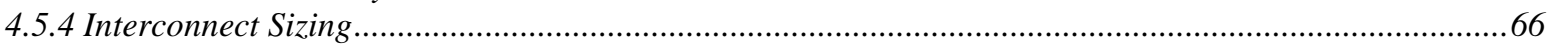

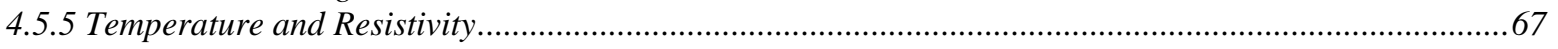

CHAPTER 5. IMPROVING DESIGN AND WAYS TO MINIMIZE FAILURE ..............................................72

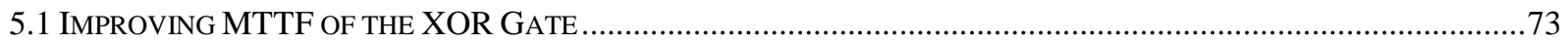

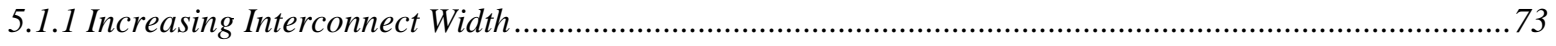

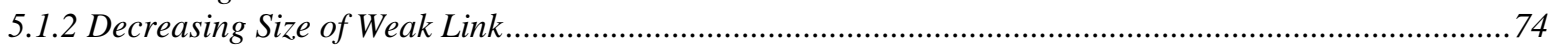

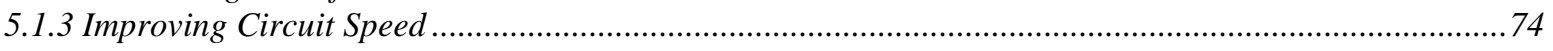

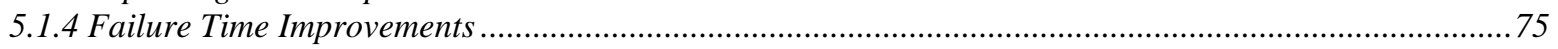

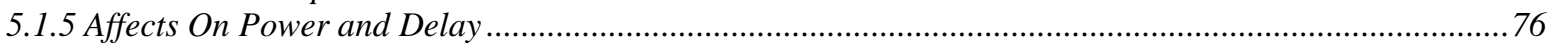

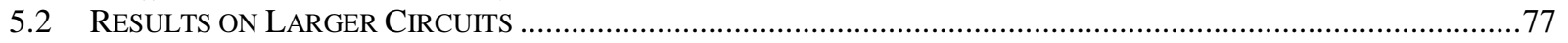

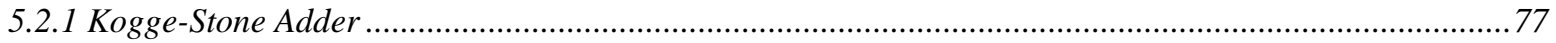

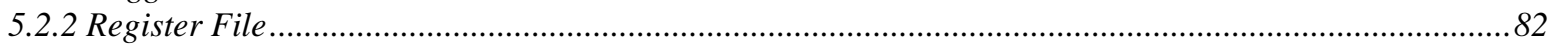

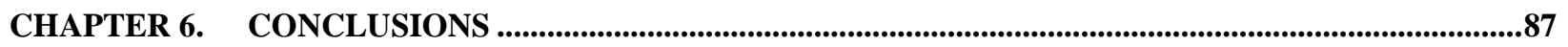

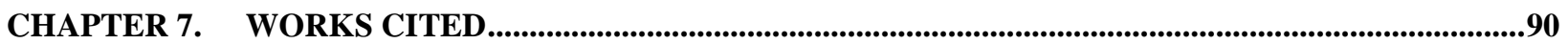

APPENDICES 93

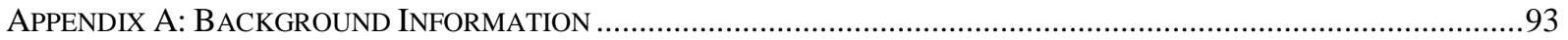

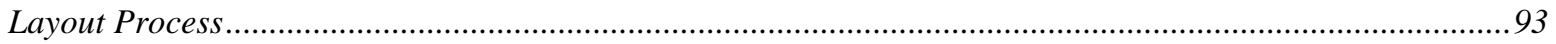

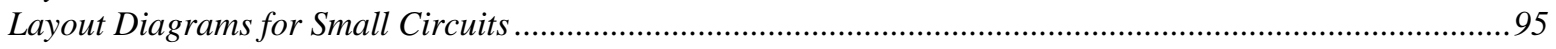

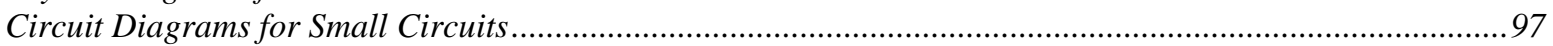

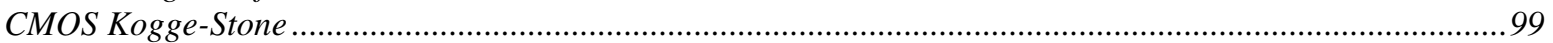

APPENDIX B: SMALL CIRCUIT EVALUATION ............................................................................................ 104

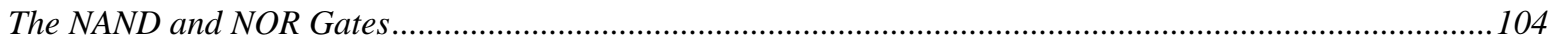

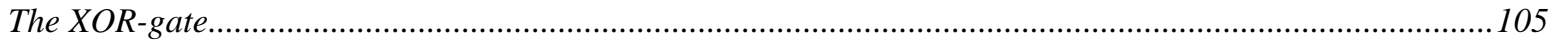

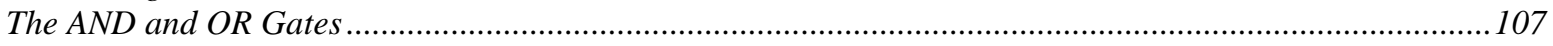

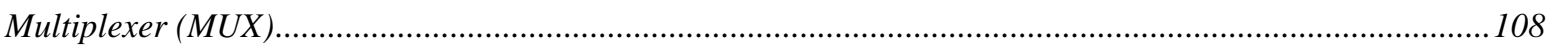

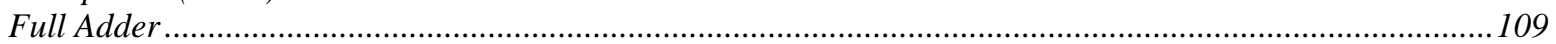

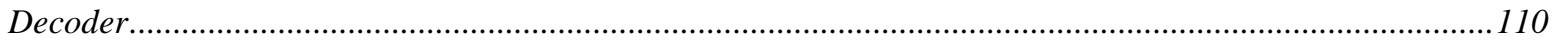

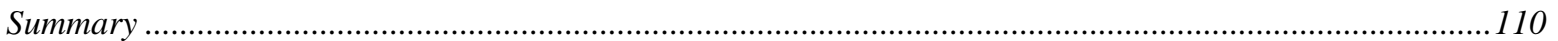

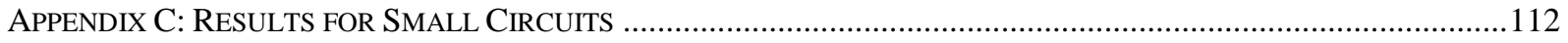

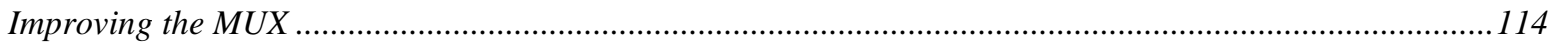

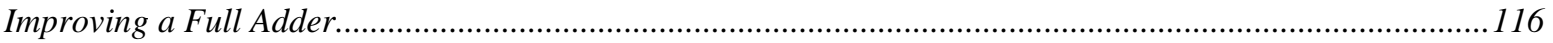

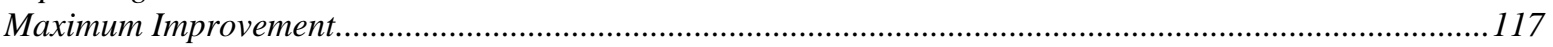

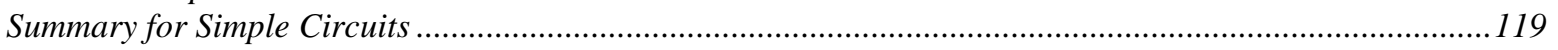

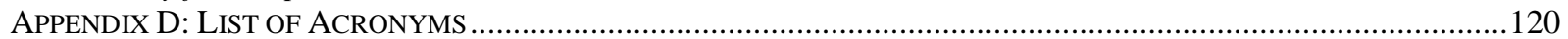

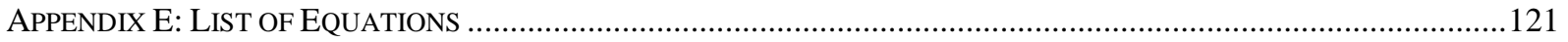




\section{LIST OF FIGURES}

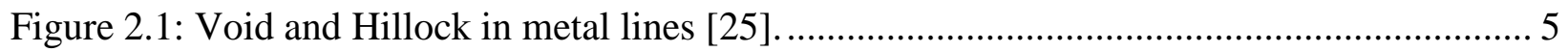

Figure 2.2: Schematic illustration of grains, grain boundaries, .................................................. 6

Figure 2.3: Thermal acceleration loop during electromigration. ............................................. 7

Figure 2.4: Temperature/Current Density Relationship.............................................................. 12

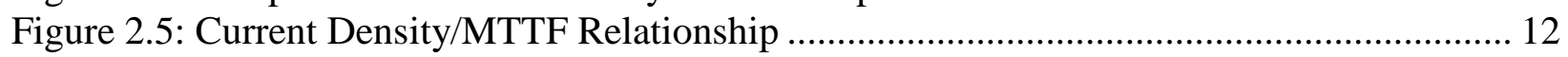

Figure 2.6: Recreated Temperature/Current Density Relationship............................................... 13

Figure 2.7: Recreated Current Density/MTTF Relationship ......................................................... 14

Figure 2.8: Reworked Current Density/MTTF Relationship for Varying Temperatures .............. 16

Figure 3.1: Overall System Block Diagram of proposed system................................................. 21

Figure 3.2: Block diagram for 16-bit Kogge-Stone prefix adder network ................................. 27

Figure 3.3: Kogge-Stone critical path of 8 logic stages for 16-bits (not all gate inputs shown) .. 27

Figure 3.4: Floorplan Representation for Hotspot .................................................................. 31

Figure 3.5: Use of Toggle tool to create floorplan files............................................................. 31

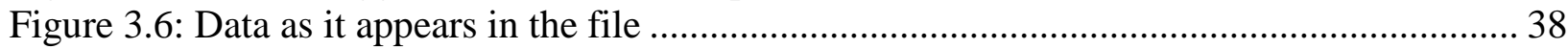

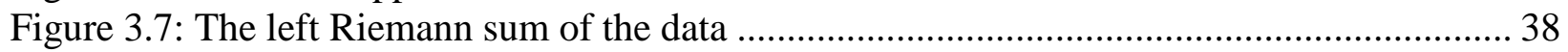

Figure 3.8: Showing method for manually finding delay ……............................................... 42

Figure 3.9: Error possibility in Delay calculations …………………........................................... 44

Figure 4.1: MTTF Improvement vs. Current Density Plot Using Created Model......................... 48

Figure 4.2: Effects of Increased Rail Voltage on MTTF........................................................... 49

Figure 4.3: Effects of Increased Rail Voltage on Current Density ............................................... 50

Figure 4.4: Effects of Temperature on MTTF for Inverter ....................................................... 51

Figure 4.5: Power vs. MTTF plot for Inverter ..................................................................... 52

Figure 4.6: Effects of Input Frequency on Power for Inverter …………………..................... 53

Figure 4.7: MTTF vs. Input Speed for Various Interconnect Sizing ……………………............5 54

Figure 4.8: Comparison of 8-Bit, 16-Bit, and 32-Bit Adders .................................................... 57

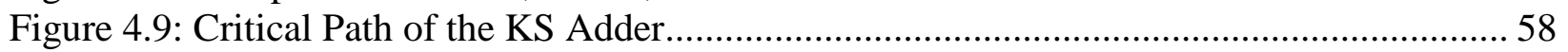

Figure 4.10: Effects of Starting Ambient Temperature, Rail Voltage and Input speed for Kogge

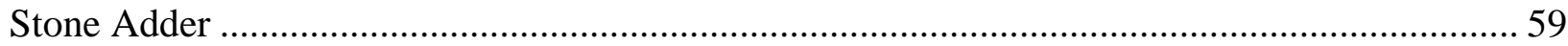

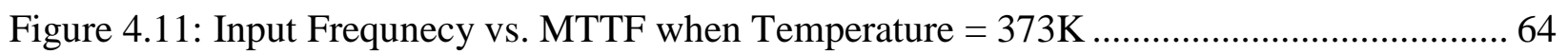

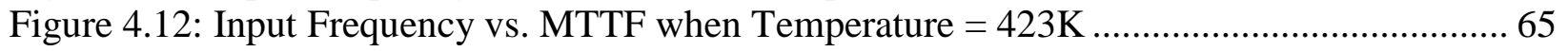

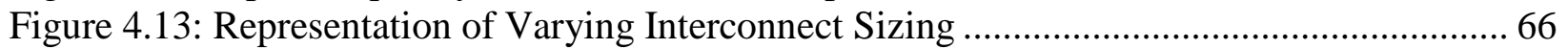

Figure 4.14: RC time delay for 8 Bit RCA ( Delay is $1.43 n s$ temp $=200$ ) .....................................6 68

Figure 4.15: RC time delay for 8 Bit RCA (Delay is $1.32 \mathrm{~ns} \mathrm{Temp}=100$ ) ……...........................6 68

Figure 4.16: MTTF vs. Input speed at Simulated and Constant Temperature (weak link XOR of

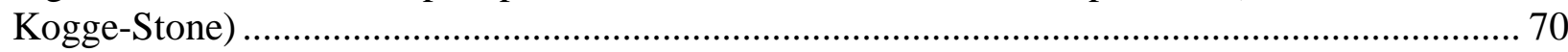

Figure 4.17: Graph Showing MTTF for Constant Current Density at Varying Starting Ambient

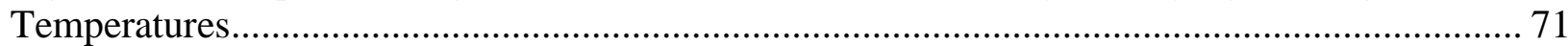

Figure 5.1: MTTF Improvement of XOR Gate for Various Changes ........................................... 76

Figure 5.2: Improvements for Subcircuits of Kogge-Stone Adder .............................................. 78

Figure 5.3: Results on Kogge-Stone Adder for Various Fixes @ 25MHz .................................... 79

Figure 5.4: Results on Kogge-Stone Adder for Various Fixes @ 400MHz …………………...... 80

Figure 5.5: MTTF Results on Register File MUX for Various Fixes......................................... 82

Figure 5.6: MTTF Results on Register File Decoder for Various Fixes ........................................ 83

Figure 5.7: Results on Register File for Various Fixes @ 25MHz................................................. 84 
Figure 5.8: Results on Register File for Various Fixes @ 400MHz...................................... 85

Figure A.1: Basic Layout of 2-input XOR gate, showing key components. ............................. 93

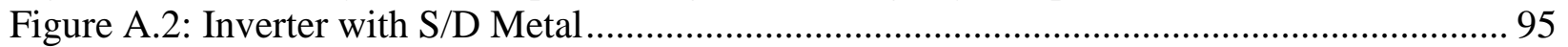

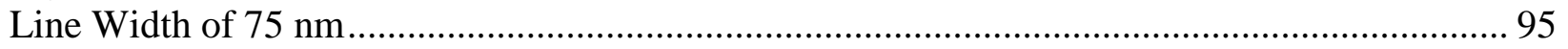

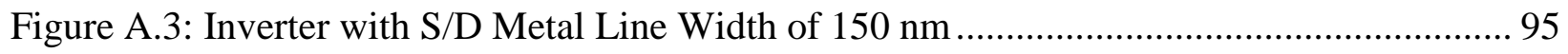

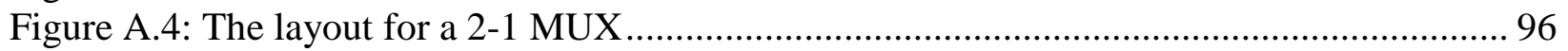

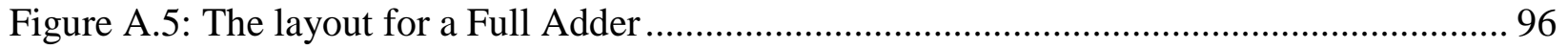

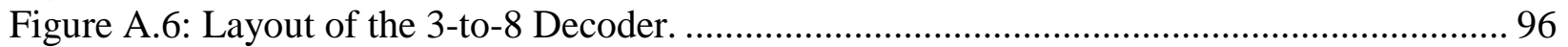

Figure A.7: Layout of a 12 Transistor SRAM Cell ............................................................. 97

Figure A.8: The Circuit for a NAND-gate (left), and a NOR-gate (right)............................... 97

Figure A.9: Transistor Network that Creates an Exclusive-OR function. ................................. 98

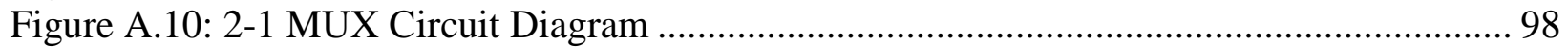

Figure A.11: Full Adder Circuit Diagram......................................................................... 99

Figure A.12: 12 Transistor SRAM Circuit Diagram ........................................................... 99

Figure A.13: Even layer tiled CMOS PPA circuit (inverted inputs, non-inverted outputs) ....... 100

Figure A.14: Even layer tiled CMOS-OPL PPA circuit layout cell ........................................ 100

Figure A.15: Odd Layer Tiled CMOS PPA circuit (non-inverted inputs, inverted outputs)...... 101

Figure A.16: Odd Layer Tiled CMOS-OPL PPA circuit cell ................................................ 101

Figure A.17: CMOS OPL layout circuits for AND (left) and XOR (right) gates used for the Pi

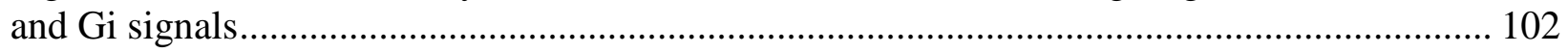

Figure A.18: Complete 16-bit Kogge-Stone Adder layout ................................................... 103

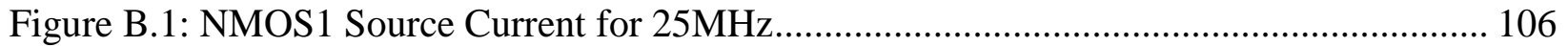

Figure B.2: NMOS1 Source Current for 400MHz............................................................ 106

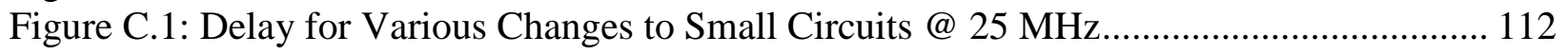

Figure C.2: Delay for Various Changes to Small Circuits @ 400 MH .................................... 113

Figure C.3: Power Consumption for Various Changes to Small Circuits at $25 \mathrm{MHz}$.............. 113

Figure C.4: Power Consumption for Various Changes to Small Circuits at $400 \mathrm{MHz}$............ 114

Figure C.5: MTTF Improvement of the MUX for various changes ...................................... 115

Figure C.6: MTTF Improvement of the Full Adder for various changes ............................... 116

Figure C.7: Overall MTTF Improvement for MUX and Full Adder...................................... 117

Figure C.8: Delay for the fixed MUX and Full Adder ..................................................... 118

Figure C.9: Power Consumption for fixed MUX and Full Adder ........................................ 118

\section{LIST OF TABLES}

Table 5.1: Comparison of Kogge-Stone fixes................................................................. 81

Table 5.2: Comparison of Register File Fixes ............................................................... 86

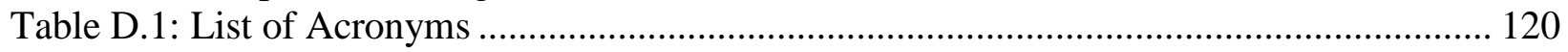




\section{Chapter 1. INTRODUCTION}

This paper will specifically address the failures in metal lines caused by electromigration, specifically the formation of voids and hillocks responsible for the destruction of the interconnect material yielding electrical discontinuity [1]. Electromigration is the transport of material caused by the gradual movement of the ions in a conductor due to the momentum transfer between conducting electrons and diffusing metal atoms. Electromigration's effects are found directly in the metal lines and are a dominating cause of digital circuit failure.

With the scaling down process of microcircuits, the effects of electromigration have become increasingly severe. Many of the subcircuits for a microprocessor are used much more than others and there are especially important subcircuits that are absolutely necessary for functionality. Binary adders and high speed memory are both an absolute necessity for microprocessors and are the most frequently used subcircuits within a microprocessor and if those fail, then the instrument that it controls fails as well.

I have researched one prominent mechanism of failure and have modified existing mathematical models that will allow me to predict the mean time to failure for these subcircuits. Upon obtaining a failure time, I will also be able to pinpoint the likely failure locations. This will give designers, device physics engineers and others valuable information early on in the design phase of complex digital circuits.

I will construct these circuits in Electric (an open-source layout tool) and PSpice and try to determine the sites of failure. I will be using HotSpot (thermal monitoring tool) for all thermal measurements and have also developed a small set of software tools that allow me to collect and massage necessary data and obtain results. 
This thesis presents a method for determining and monitoring the failure of complex digital circuits caused by electromigration. Furthermore, this thesis specifically addresses the electromigration failure type and shows the development of a system that can estimate the failure time and likely failure locations due to electromigration, ideally down to the transistor level. Overall system architecture and test methodology will be discussed.

Chapter two of this thesis presents additional background material about electromigration and its effects. Section 2.1 describes the three predominant failure mechanisms associated with electromigration, those being the metallurgical statistical properties of the conducting film, the thermal acceleration process and the healing effects. Section 2.2 discusses past research on electromigration and the associated methods for estimating failure time. Finally, section 2.3 concerns the creation of a mathematical model to predict circuit lifetime.

Chapter three outlines the actual system implementation and the requirements for the system. Section 3.1 provides a general description of the tool chain and the goals it seeks to achieve. Section 3.2 goes over the system requirements and the necessary tools the system needs to be successful. Section 3.3 gives an overview of the layout process, and also describes the various circuits used in the tool chain. Section 3.4 concerns the creation of various intermediate files for the system. Section 3.5 describes "the program", which joins all system components together, and finally, section 3.6 discusses the few additional tools needed before the system can be implemented.

Chapter four contains all the circuit evaluation work, discusses exactly what happens and why and then pinpoints failure locations for the various circuits. Section 4.1 describes the testing process, while section 4.2 verifies the created failure model. Section 4.3 shows initial test results 
for an inverter, and section 4.4 shows that for complex circuits. Section 4.5 goes into detail on the actual performance flaws of the circuits and outlines areas of improvement.

Chapter five addresses ways to improve design and minimize failure time. Section 5.1 shows improvement techniques for the XOR gate, and section 5.2 shows improvement techniques for larger circuits, like the Kogge-Stone adder and 8x8 register file.

Chapter six summarizes the project, briefly restating the causes for electromigration and the methods to minimize its effects based on the findings within the paper. The set of Appendices include background information, a list of terms and equation, and circuit and layout diagrams. Also, small circuit evaluations and results can be seen here. 


\section{Chapter 2. BACKGROUND}

Prior to discussing the mathematical models created, and the implementation of my method for locating failures, this chapter will provide some basic definitions and theory related to electromigration and the system's operation. As mentioned earlier, these tools attempt to diagnose both simple and complex circuits in detail, predict failure times and pinpoint the locations of those failures. This background will specifically discuss the definition of electromigration and associated failure mechanisms, past research in the area, and finally the creation of the mathematical model used for determining failure time.

As microcircuits are scaled down, the density of electric current in interconnecting metal lines increases, as does the temperature of the actual device. Electromigration is generally considered to be the result of momentum transfer from the electrons, which move in the applied electric field, to the ions which make up the lattice of the interconnect material [3][4]. The metallic atoms constructing the line are transported by an electron wind. Under these conditions, electromigration can lead to the electrical failure of interconnects in relatively short times, reducing the circuit lifetime to an unacceptable level. It is therefore of great technological importance to understand and control electromigration failure in thin film interconnects.

The damage induced by electromigration appears as the formation of voids (which occur along the length of the line) and hillocks (electrons "push" the metal atoms in direction of current). With the growth of these voids in the metal lines, electrical discontinuity arises [5][6]. Recent research has shown that both of these failure modes are strongly affected by the microstructure of the line and can, therefore be delayed or overcome by metallurgical changes that alter the microstructure. 


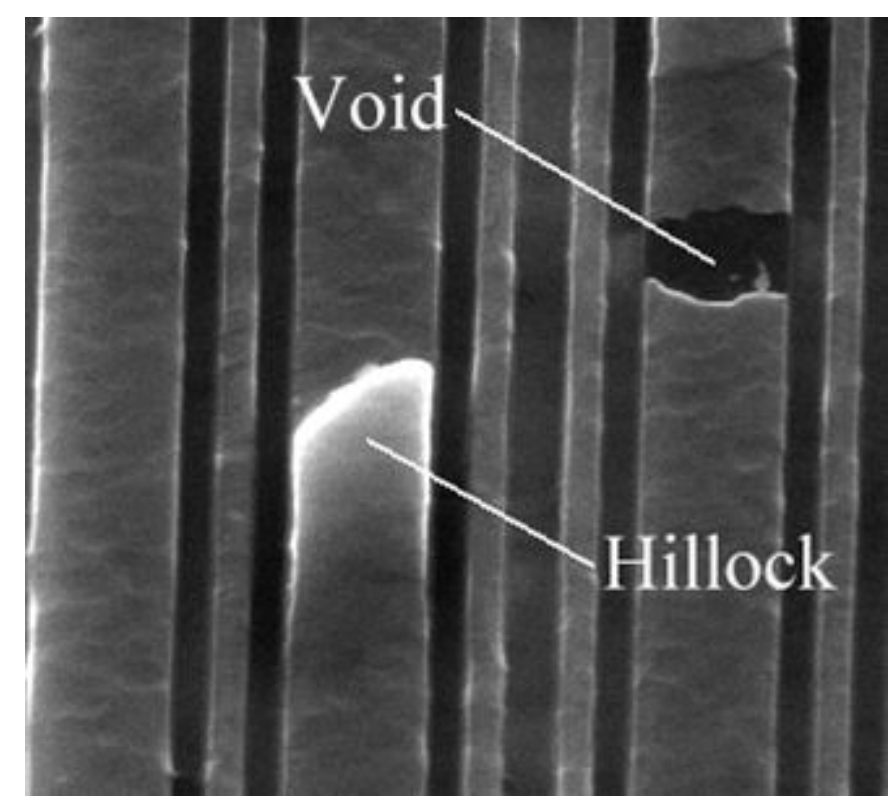

Figure 2.1: Void and Hillock in metal lines [25].

Above is a picture under a microscope showing both a void and hillock inside a metal line. Both are effects of electromigration. Because of high current densities in the metal interconnects, the electrons 'push' the metal atoms in the direction of the current. Voids at one end of the metal line, and bumps (Hillocks) at the other end are the result of this electron wind.

\subsection{Failure Mechanisms of Electromigration}

There are three predominant failure mechanisms in the electromigration process. They are (a) the metallurgical statistical properties of the conducting film, (b) the thermal acceleration process and (c) the healing effects. Here, they will be explained in more detail.

\subsubsection{Metallurgical Statistical Properties of the Conducting Film}

The metallurgical properties of a conductor film refer to the microstructure parameters of the actual conductor material, including grain size distribution, and the distribution of grain 
boundary misorientation angles, and the inclinations of grain boundaries with respect to electron flow [1][9].

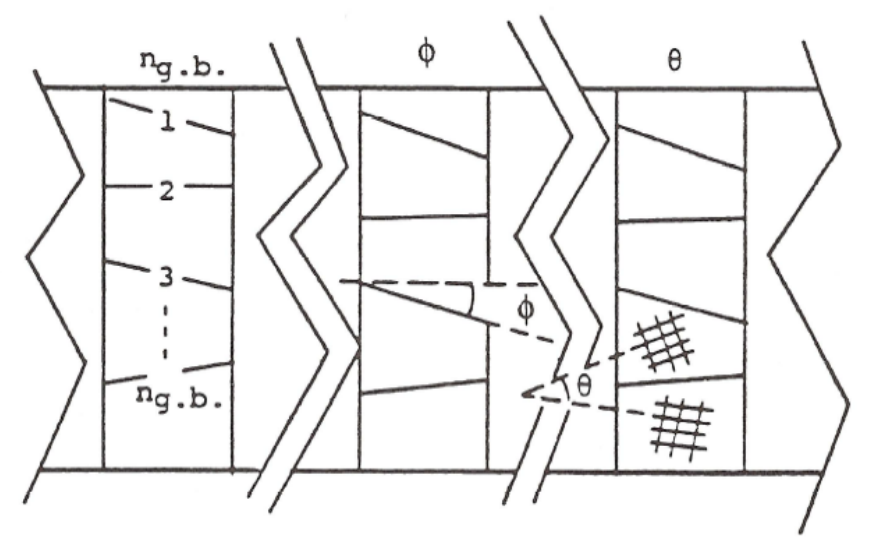

Figure 2.2: Schematic illustration of grains, grain boundaries, grain boundary misorientation angles, and inclination angles. Aftger Nikawa Kiyoshi, IEEE Int. Reliab. Phys. Symp., CH1619-6, 175 (1981). ( ) IEEE 1981.

Because these parameters appear to be random, they can only be dealt with statistically. As illustrated in the figure, the misorientation angle, $\theta$, between the two grains defining the grain boundary determine the mobility of atoms in that boundary.

It is also important to look at the grain boundary inclination with respect to electron flow, $\varphi$, partially determined by grain size variation, for what $\square$ determines the effectiveness of the applied field for that grain boundary; finally, the grain size variation determines the change in the number of atomic paths across a cross section of the conductor line. The variation of above parameters over a film leads to a nonuniform distribution of atomic flow rate. As a result, a nonzero atomic flux divergence exists at the places where the number of atoms flowing into the area is not equal to the number of atoms flowing out (6). If divergence is greater than zero, there is mass depletion, and if the divergence is less than zero, there is mass accumulation, which leads to voids or hillocks [1] [10]. 


\subsubsection{Thermal Acceleration Process}

The second failure mechanism is the thermal acceleration process, which refers to the acceleration process of electromigration damage due to the local temperature rising. It is only possible to have a uniform temperature distribution before electromigration damage occurs. Immediately following the formation of a void, the current density increases (current crowding) in its vicinity as it reduces the cross sectional area of the conductor. Joule heating is proportional to the square of current density, which as a result, leads to a local temperature increase around the void, accelerating the voids growth. This process goes on until the void is large enough to break the line [3] [6]. The thermal acceleration loop is shown in the figure below.

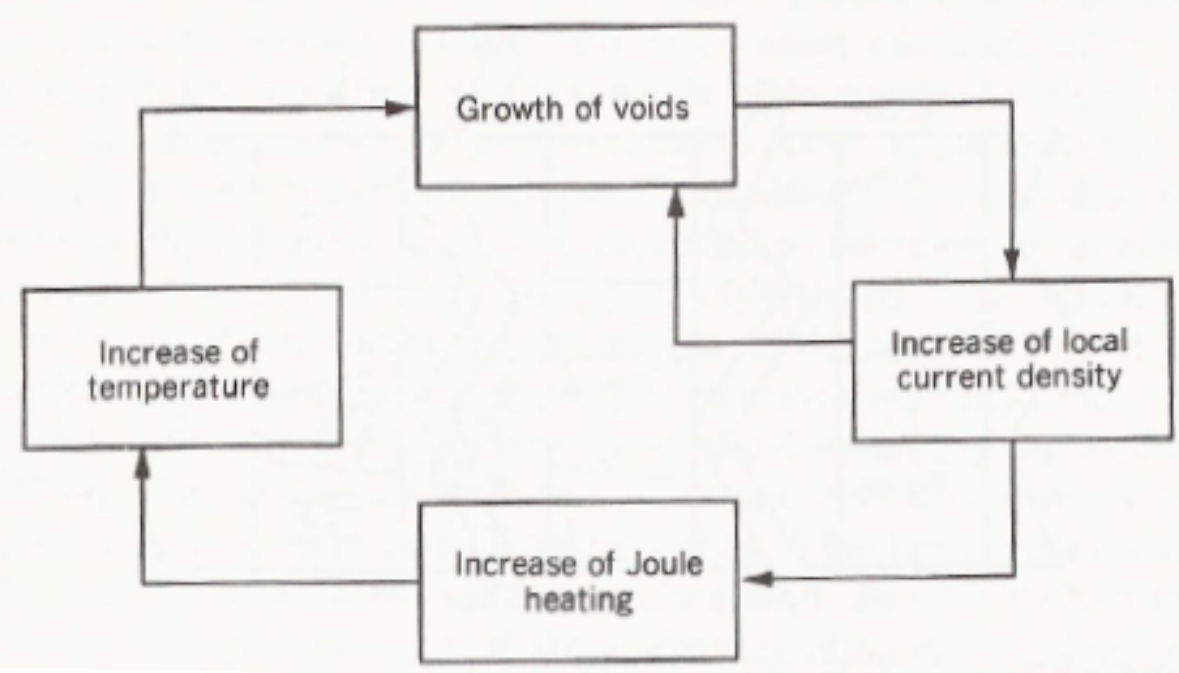

Figure 2.3: Thermal acceleration loop during electromigration. Aftger Nikawa Kiyoshi, IEEE Int. Reliab. Phys . Symp., CH1619-6, 175 (1981). () IEEE 1981.

When the heat dissipation through the film substrate is poor, the thermal run-away process often becomes the domination electromigration failure mechanism. Also, because of the lack of control on temperature uniformity, a temperature gradient may exist along a conductor line even before 
the formation of damage. This temperature gradient itself can lead to a nonuniform flux divergence (mobility of atoms depends on this), thus in order to model electromigration failure properly I must have an accurate temperature determination along the conductor film.

\subsubsection{Healing Effects}

The last of the failure mechanisms is the healing effects, specifically those caused by the atomic flow of electrons opposite in direction to the electron wind [6]. This backflow of mass is caused by things such as temperature and/or concentration gradients that result from electromigration damage. The system is essentially aggravated from its stable state. It is initiated once a redistribution of mass has begun to form. This helps in reducing the failure rate during electromigration and somewhat heals the damage after the current is removed. Because of this mass backflow, there exists a threshold density for electromigration to become effective. This value corresponds to a minimum energy barrier that the atoms have to overcome to balance off the backflow driving forces. It is important to understand these failure mechanisms so one can possibly reduce electromigration effects.

\subsection{Past Research}

The sections below briefly describe some past research and techniques used in estimating the effects of electromigration. The first is known as Black's equation and dates all the way back to 1969 . The second method is known as numerical simulation, while the last method dives into extreme detail on the actual physics behind electromigration. All are good methods for obtaining realistic data pertaining to electromigration and its effects.

My method however, creates a new model for calculating MTTF and pinpointing metal failures while being very user friendly. From a user standpoint, any catalog of laid-out circuits 
can be used. There is a bit of manual labor that must be done in creating what's known as a floorplan, but this will all be discussed in chapter three. The software tools do most of the work and keep track of the majority of data. The user must make some minor tweaks to circuits while testing for improvements, but they are done easily and then dumped back into software.

\subsubsection{Black's Equation}

J.R Black was the first to develop an empirical formula for calculating the MTTF (mean time to failure) of a semiconductor circuit due to electromigration. The equation is as follows (J.R Black - electromigration) [14][15]:

$$
M T T F=A w j^{-n} e^{\left(\frac{Q}{k T}\right)}
$$

Equation 2.1

Here, $A$ is a constant, $j$ is the current density, $n$ is a model parameter, $Q$ is the activation energy in $\mathrm{eV}$ (electron volts), $k$ is Boltzmann constant, $T$ is the absolute temperature in $\mathrm{K}$, and $w$ is the width of the metal wire. The model is abstract, not based on a specific physical model, but flexibly describes the failure rate dependence on the temperature, the electrical stress, and the specific technology and materials. The values for $A, n$, and $Q$ are found by fitting the model to experimental data. This is not a universal method for determining the lifetime of a circuit, as many long term experiments are necessary for the respective line shapes, despite the fact that the lines are made of the same metallic film.

\subsubsection{Numerical Simulation}

The conventional method in determining failure location is Numerical Simulation [16]. The main purpose of this is to clarify the damage mechanisms. There was also a way in which to 
evaluate threshold current density, $j_{t h}$, yet this assumed that the product of threshold and line length was constant [16]. This is simple and easy, however the effect of line shape on $j_{t h}$ is not considered (Application is limited to only straight line). The constant is also temperature dependent.

\subsubsection{Atomic Flux Divergence}

More recently, there was a unified approach to dealing with electromigration based on atomic flux divergence (AFD). The AFD-based simulation of failure process [2][9] is universal. Once the film characteristic constants are obtained, the failure prediction of any shaped line is possible under arbitrary operating conditions. This allows for an accurate prediction for not only lifetime but also failure site. Second, the AFD-based simulation of electromigration behavior [2] shows that AFD corresponds with actual amount of damage. Film characteristic constants can be derived by simple experiments to measure the amount of damage. Finally, there is the AFDbased simulation for building-up process of atomic density distribution or what is called the incubation period. This too is universal and accurate, as once the film characteristic constants are obtained, the evaluation of the threshold in any shaped line is possible under arbitrary temperature [2].

\subsection{Creation of a Mathematical Model}

\subsubsection{Calibration}

Because circuits were not fabricated for this project, Black's equation as well as the numerical simulation and AFD methods for determining failures time can only be used as guidelines. The constraints and variables cannot be determined experimentally, and require more 
of a device physics approach to implement. Thus, the creation of my own mathematical model was necessary. Using past research as a guideline, and combining this research with data from a variety of papers, a legitimate model was formed. The two biggest factors in obtaining an accurate MTTF model are temperature and current density of the circuit. However, there is no direct correlation between MTTF, temperature and current density. A variety of papers show relationships between any two of these.

Data points from these papers were plotted in Microsoft Excel and an exponential relationship between them was found using some extrapolation. For example, one paper [25] shows the relationship between current density and temperature, while another [26] shows the relationship between current density and MTTF. Because of the ongoing debate as to which model is the most accurate, this method is just as valid, and the created model can be used.

Again, one major drawback to this is that there is not a purely mathematical relationship between failure time, temperature and current density. In figure 2.4 [25] the data represents a graphical relationship between temperature and current density. Notice that in the figure, the temperature range is between 200 and 230 degrees Celsius. For this project, high temperature is assumed, as electromigration does not take affect at low temperature values. As can be seen, the relationship is clearly exponential and temperature sharply increases with the higher current densities. For example, the difference in temperature between current densities of $1.1 \times 10^{6}$ and $2.1 \times 10^{6}$ is only a few degrees. Yet the difference between $3.1 \times 10^{6}$ and $4.1 \times 10^{6}$ is much more significant. This relationship is found in a variety of papers, yet none of the basis use it the basis for a model. 


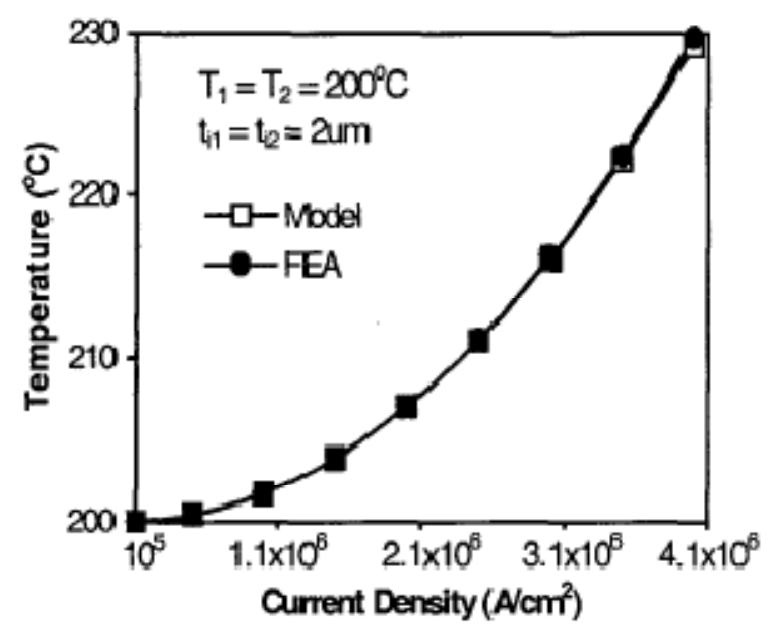

Figure 2.4: Temperature/Current Density Relationship

Figure 2.5 [26] shows a plot of normalized MTTF vs current density. In this graph, the MTTF is adjusted to a logorithmic scale to show a more exponential relationship. Again, the MTTF is not greatly affected until the higher densities, which in turn, yield an increase in temperature.

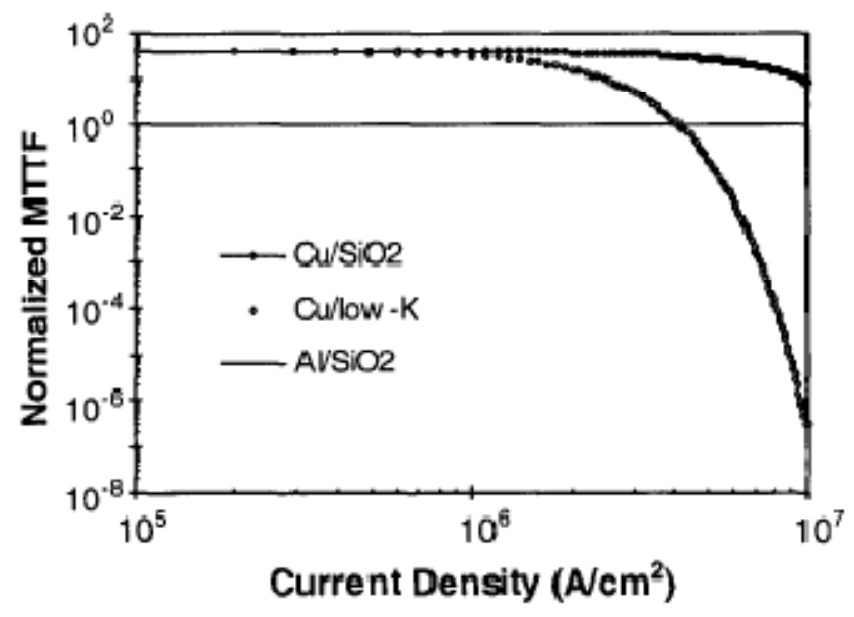

Figure 2.5: Current Density/MTTF Relationship

The data represented in this paper concerns different interconnect types. For my purposes, I will focus on the $\mathrm{Cu}$ /low $\mathrm{k}$ dielectrics. Both papers show the authors' findings for a single MOSFET. 
In order to formulate a model that included a relationship between all three variables, the data from both papers was combined. I first recreated each of the plots in Microsoft Excel and found the individual exponential relationships for each. Figure 2.6 below shows the plot and resulting equation.

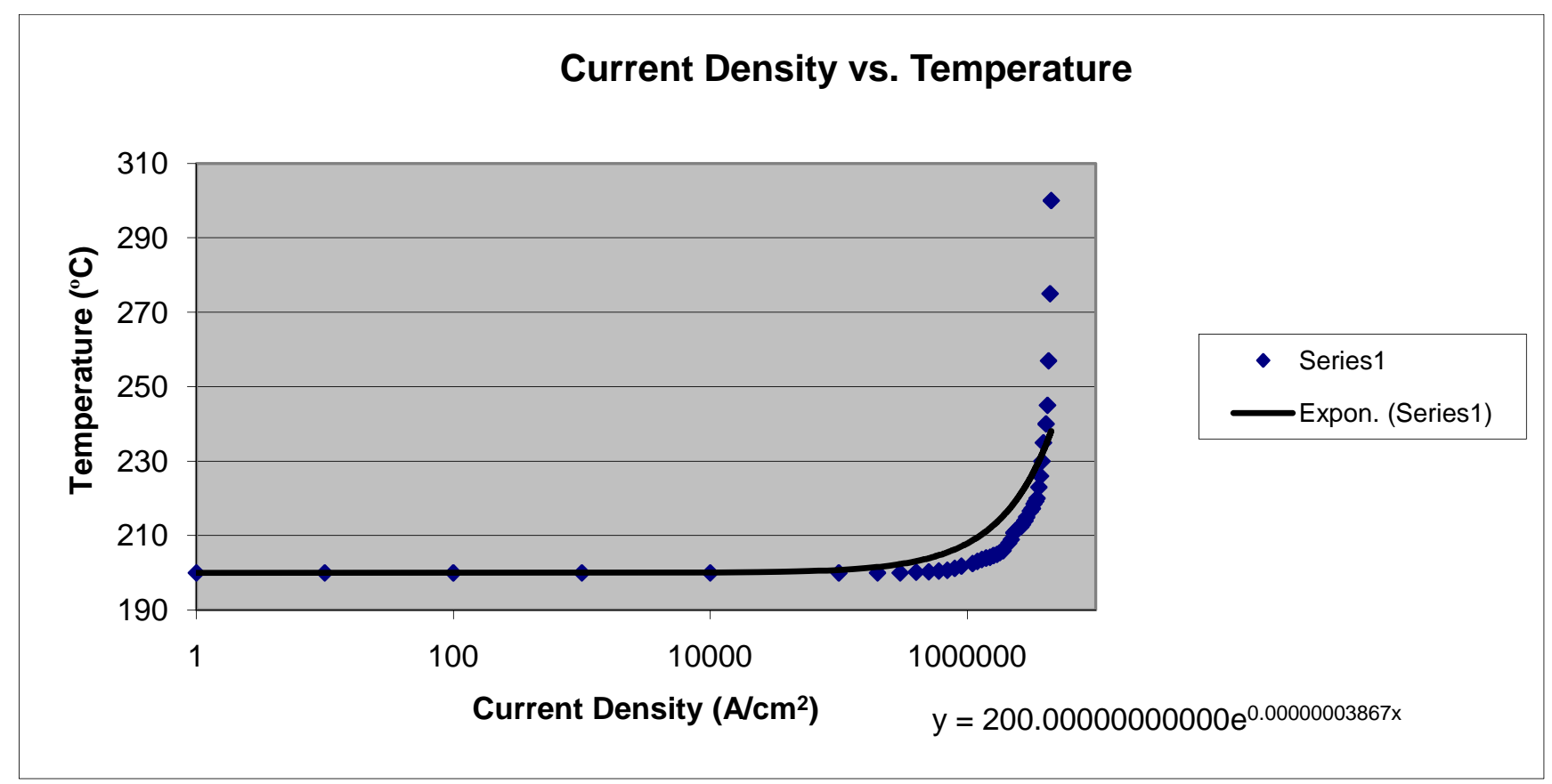

Figure 2.6: Recreated Temperature/Current Density Relationship

For purposes of this paper, current density will be known as $\mathrm{J}$ and temperature as $\mathrm{T}$. The resulting equation is thus $\mathrm{T}=200 \mathrm{e}^{3.867 \mathrm{E}-08 \mathrm{~J}}$. Several extra data points not shown in the paper were added to create a more accurate equation. Figure 2.7 below shows the resulting plot and equation. 


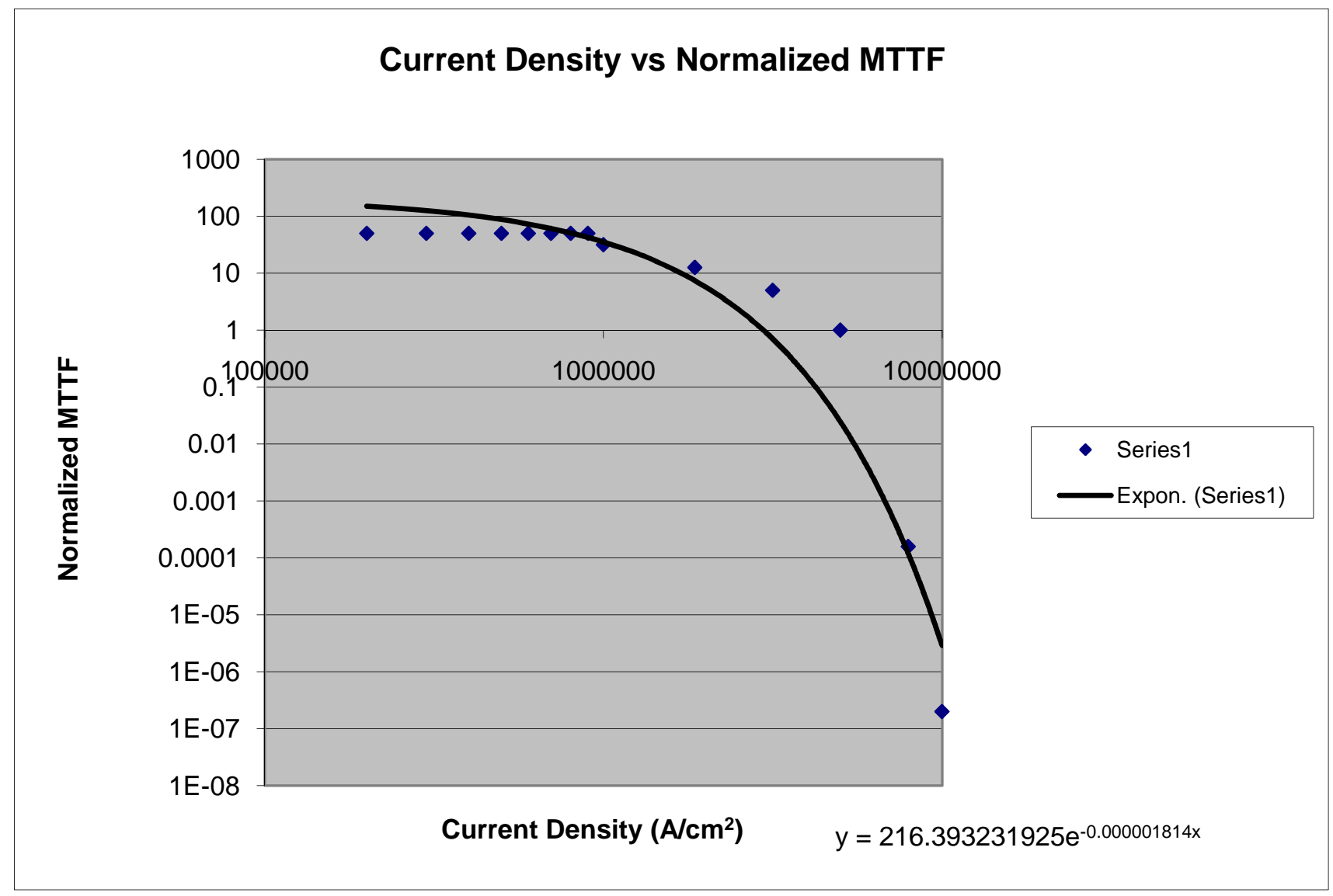

Figure 2.7: Recreated Current Density/MTTF Relationship

Here, the relationship between MTTF and $\mathrm{J}$ is MTTF $=216.39 \mathrm{e}^{-1.814 \mathrm{E}-06 \mathrm{~J}}$. Now I have two clear relationships between the various factors affecting failure time. I still do not have an equation however that considers all three variables. With some simple algebra, I can add the two equations together, and the result is an MTTF equation dependant on $\mathrm{J}$ and T.

$$
M T T F=200 * e^{(3.867 E-08 * J)}+216.39 * e^{(-1.814 E-06 * J)}-T
$$

\section{Equation 2.2}

Although this equation expresses MTTF as a function of current density and temperature, it is not entirely finished. When T becomes very large, the resulting MTTF is negative, which poses a 
problem. To address this issue, the equation was recreated for four fixed temperatures. Because I decided to simulate my circuits at high temperatures, the equation uses 100, 150, 200, and 250 degrees Celsius for the fixed $\mathrm{T}$ values. The equations are shown below:

$$
\begin{gathered}
M T T F=200 * e^{(3.867 E-08 * J)}+216.39 * e^{(-1.814 E-06 * J)}-100 \\
\text { Equation 2.3 } \\
M T T F=200 * e^{(3.867 E-08 * J)}+216.39 * e^{(-1.814 E-06 * J)}-150 \\
\text { Equation 2.4 } \\
M T T F=200 * e^{(3.867 E-08 * J)}+216.39 * e^{(-1.814 E-06 * J)}-200 \\
\text { Equation 2.5 } \\
M T T F=200 * e^{(3.867 E-08 * J)}+216.39 * e^{(-1.814 E-06 * J)}-250
\end{gathered}
$$

\section{Equation 2.6}

From these four equations, I can vary current density for each of the fixed T values. Only high current density values were used as low values of $\mathrm{J}$ have little effect on $\mathrm{T}$, thus minimal effect on MTTF. This will then give me new data with four different relationships between MTTF and current density at the four chosen $\mathrm{T}$ values. These equations are shown below:

$$
\text { For } T=100: M T T F=308.3401 e^{-0.000001089 J}
$$

Equation 2.7

$$
\text { For } T=150: M T T F=260.6282 e^{-0.000001385 J}
$$

Equation 2.8

$$
\text { For } T=200: M T T F=215.0316 e^{-0.000001906 J}
$$

Equation 2.9

$$
\text { For } T=250: M T T F=177.1201 e^{-0.000002996 J}
$$

\section{Equation 2.10}




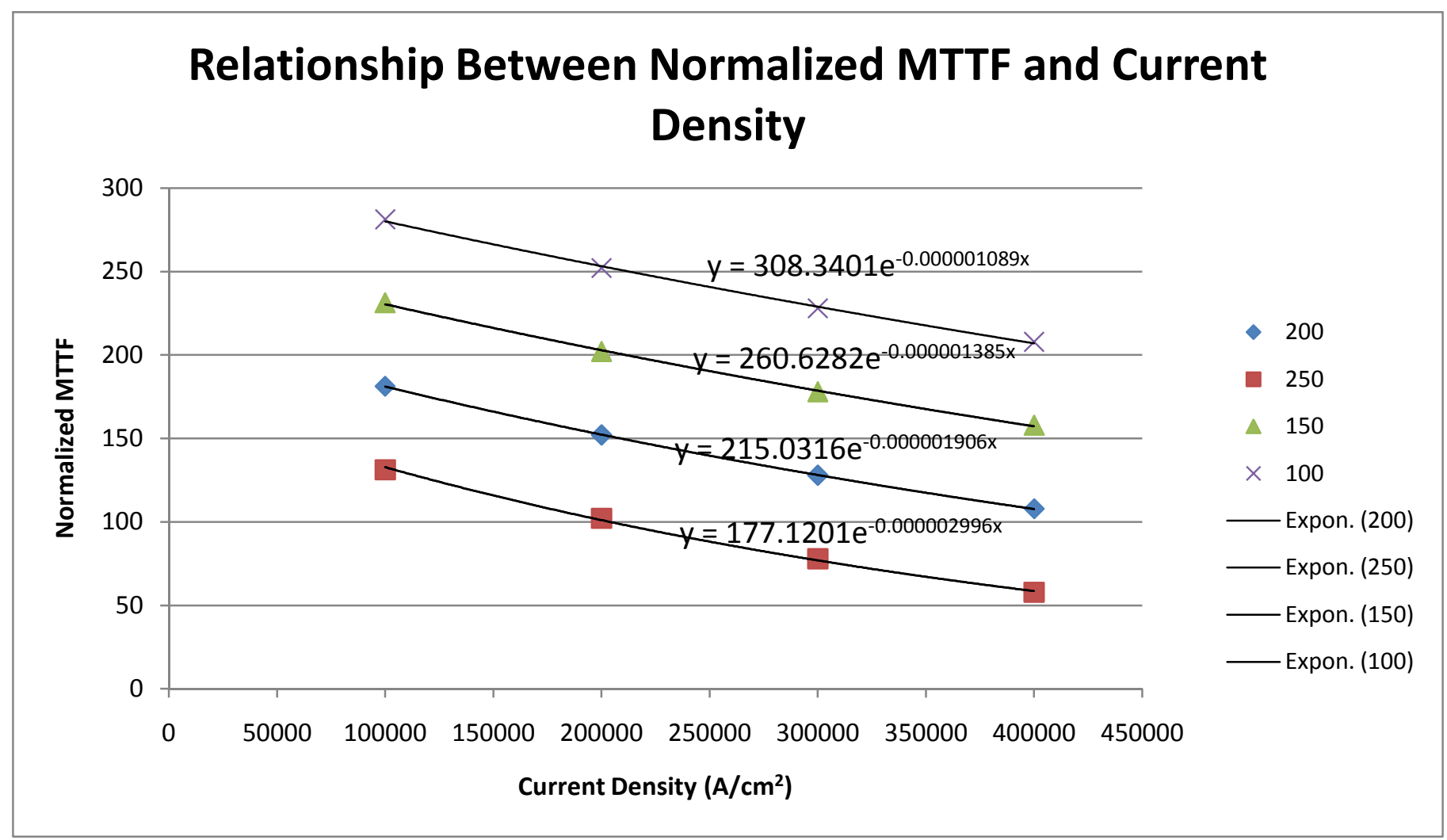

Figure 2.8: Reworked Current Density/MTTF Relationship for Varying Temperatures

I can now modify these equations a bit in order to get any of the three equations from any single equation. To make this easier to see, below is an algebraic method of how it's done.

For example, to get any of the other 3 equations from that at $\mathrm{T}=100$, I can use the following model,

$$
\left(308.3401+N\left(100-T_{S}\right)\right) e^{\left(-1.089 E-06+M\left(100-T_{S}\right)\right) J}
$$

Equation 2.11

where $\mathbf{T}_{\mathbf{s}}$ is the temperature for the desired equation. So, if I wish to get the equation for $\mathrm{T}=150$ from the above model, my method for solving for $\mathrm{N}$ and $\mathrm{M}$ is as follows: 


$$
(308.3401+N(100-150)) e^{(-1.089 E-06+M(100-150)) J}=260.6282 e^{-0.000001385 J}
$$

Equation 2.12

For $\mathrm{T}=200$, my method is as follows:

$$
(308.3401+N(100-200)) e^{(-1.089 E-06+M(100-200)) J}=215.0316 e^{-0.000001906 J}
$$

Equation 2.13

And for $\mathrm{T}=250$, my method is as follows:

$$
(308.3401+N(100-250)) e^{(-1.089 E-06+M(100-250)) J}=177.1201 e^{-0.000002996 J}
$$

Equation 2.14

Now I have three equations and two unknowns, and with a bit of algebra, $\mathrm{N} \approx 0.9207$ and $\mathrm{M} \approx$ 8.9344E-09. Using these values, I can obtain a generic equation for MTTF at any temperature. This will allow me to now model my circuits and find some interesting relationships between MTTF, current density, and temperature. My model is to be used in the circuit test infrastructure and the wear-out model is very simple to update when appropriate. Like all models, there are some drawbacks. This model is great for estimating failure time, but cannot be used for exact measurements. The model was created to simply show a distinct relationship between failure time, current density, and temperature with the capability of estimating failure times with known parameters.

\subsubsection{How is the Model Used}

Now that I have established a useful model for obtaining the MTTF of a given circuit, I can explain how the exactly the software uses the model. The model relies on fixed values for both current density and temperature. As you will see in the following chapter, I have created a monitoring program needed to handle all the data outputted from LTSpice. One of the 
monitoring program's duties is to track all gate, source, and drain current for each transistor in any given circuit. Attached to each source and drain are metal lines through which the current travels. Current density can be calculated using the following equation:

$$
J=\frac{A}{w * h}
$$

Equation 2.15

Where $\mathbf{A}$ is the total current through a particular metal line, and $\mathbf{w} * \mathbf{h}$ refers to the cross sectional area of the metal wire, $\mathbf{w}$ being the width of the line and $\mathbf{h}$ being the height. Current density will be measured as Amps/cm^2.

Temperature is handled through Hotspot. Hotspot is an accurate and fast thermal model suitable for use in architectural studies. Given power dissipation with displacement information, HotSpot can calculate the steady state temperature of a region within a circuit and calculate the affect of a particular region's temperature on other regions.

Current density and temperature for all circuits are now taken care of. The model for MTTF can thus be used to find an accurate failure time for a given circuit, beginning with the basic inverter and expanding to more complex circuits. 


\section{Chapter 3. SYSTEM DESCRIPTION}

This chapter addresses the implementation/system architecture, choice of circuits, and the software tools, both readily available and created, to handle failure calculations. The system requirements and overall system goals are also discussed.

\subsection{General Description}

My overall goal is to find the MTTF impact of EM on digital circuits. The mathematical model used for estimating failure times was done by pulling information and data from a variety of technical papers. The data was then recreated in MS Excel, and an equation was formed by joining together the various data collections. The key variables in the equation are essentially MTTF, current density, and temperature. Their definitions and relationships are explained in chapter two. Finding relationships between all three of these variables was essential before forming an accurate equation.

The tool chain I designed required a general model for MTTF, VLSI plots, SPICE models and several operating conditions as inputs. The chain returns a file that shows the MTTF of various circuit nodes for a given input. I will test both simple and complex circuits. Starting with the very basic transistor, inverter and simple gates, and then creating more complicated highpriority circuits. I will create a few of the important pieces to any microprocessor, including decoders, adders and some memories.

After creating a decent size catalog of digital subcircuits to test, I designed and implemented a program that monitors the relative usage of metal lines and transistors, pinpoints the weakest links in the circuit, and ultimately estimates failure time. My program needs a UNIX as well as a MS Windows environment in order to take advantage of the many tools needed for my failure analysis. 
After the program is up and running, the circuits are ready for testing. The circuits will be evaluated and the program will dump the results into a spreadsheet for analysis. The spreadsheet contains failure time calculations, current density measurements, etc. Once I have located the weakest links in the design, I will be able to alter the design and see if it can be improved.

\section{Assumptions:}

- Perfect fabrication of circuits. Errors in fabrication will lead to quicker MTTF for MOSFETS.

- The circuits are isolated from the outside world. Thermal transfer is valid within the given circuit and does not assume outside heat sources except ambient temperature

- Most of the power dissipated in a circuit is from the MOSFETs and not the metal lines, so when choosing a region, originally, a region of a floor plan was based solely on the gate of the MOSFETs, but because of HotSpot's minimum size limits for a region, gates (i.e. NAND/NOR/etc) rather than individual FETs became the regions of the floor plan.

\subsection{System Overview and Requirements}

The project contains a mix of theory, hardware and software. As a general implementation strategy, the system adheres to the block diagram in Figure 3.1. 


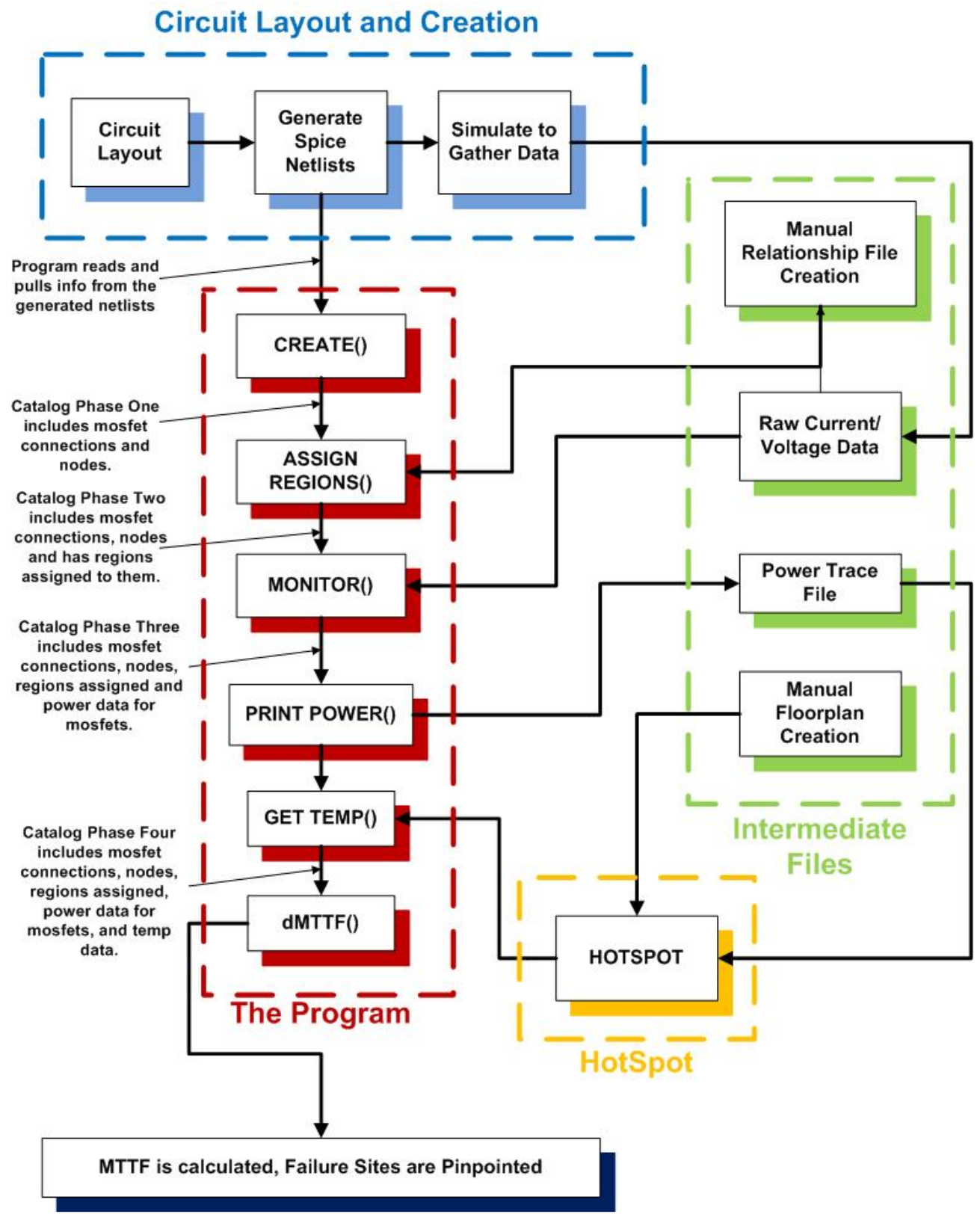

Figure 3.1: Overall System Block Diagram of proposed system.

This top-level diagram depicts the hardware/software implementation strategy of my method for evaluating wear-out. The system consists of 4 major sections, which comprise of the circuit layout/creation, the program, various intermediate files, and HotSpot.

The proposed system consists of 4 major sections. They are circuit layout/creation, the program, various intermediate files, and Hotspot. The following subsections contain a general 
description, and theory, of operation for the 4 major sections. Also, the readily available design tools used in the system, as well as those I created are briefly explained.

The basic equation for mean time to failure is $308.3401+0.9207 *\left(100-\mathrm{T}_{\mathrm{s}}\right) * \mathrm{e}^{\wedge}((-1.089 \mathrm{E}-$ $\left.\left.06+8.9344 \mathrm{E}-09 *\left(100-\mathrm{T}_{\mathrm{s}}\right)\right)^{*} \mathrm{~J}\right)$. With that in mind the two main things that the monitoring program has to extract from a circuit are current density and a temperature. Unfortunately these values cannot readily be determined from looking at a schematic or layout of a circuit. Many tools are needed to discover those quantities, some of which are readily available and others that must be created. The tools used for this project are:

\subsubsection{Design Tools Used in the System}

\section{$\underline{\text { Electric VLSI }}$}

Electric is a VLSI program that creates circuits that has the information of both the placement of specific components such as wires and MOSFETs as well as the realistic capacitance of a circuit. This program is important because the placement of a component can determine the amount of heat transfer to other components as well as the capacitance added by wires can help to create a more realistic circuit. It is open source and is a very powerful layout utility.

\section{$\underline{\text { LTSpice }}$}

LTSpice is a high performance Spice III simulator. LTSpice is used to simulate the circuit and provide the appropriate voltage and current data necessary to calculate a proportional electric field as well as the power necessary to calculate temperature. 


\section{$\underline{\text { LTSpice Utility }}$}

This is a utility for LTSpice that has many functions, but for the purposes of this project, it is used to convert the raw binary data files that LTSpice outputs to an ASCII format that can be used by the monitoring program.

\section{$\underline{\text { HotSpot }}$}

Hotspot is an accurate and fast thermal model suitable for use in architectural studies. Given power dissipation with displacement information, HotSpot can calculate the steady state temperature of a region within a circuit and calculate the effect of a particular region's temperature on other regions.

\subsubsection{Software Tools I've Created}

\section{Monitoring program}

A program is necessary to monitor all the data output from LTSpice. The program also needs to know the various regions within the circuit as well as where each individual MOSFET is located. Also the program calls HotSpot and once all the data is correlated, it determines a relative mean time to failure for Electromigration. In the later sections this will be known as "the program."

\section{$\underline{\text { Simple scripts }}$}

In order to more efficiently run the necessary program, scripts were created to format information, modify files, or test for speed. These tasks could be done by hand, but doing so would be too tedious and would waste time. 
The entire tool chain runs in a Linux/Unix environment, specifically Ubuntu. The main reason for this is because the Unix environment is ideal for creating, compiling, and running $\mathrm{C}$ programs and Perl scripts. One of the main reasons for use of Ubuntu is unfamiliarity with programming in a Windows environment.Also in order to make the program easier to use for the user, some of the capabilities of Unix programming such as the ability to call other programs and redirect its output back to the calling program are used.

\subsection{Circuit Layout and Creation}

The section below describes the first major piece of my system. This section discusses the choice of circuits used, the technology used to create them, layout preferences, and the generation of spice netlists. I wanted to create a wide range of circuits, some being as basic as individual gates, while adders and memories remain more complex. Although electromigration failures are only likely to be seen in the larger more complicated circuits, the mathematical models and software developed must cater to any size circuit. Regardless of the complexity in a design, failure times and locations for any size circuit can then be found. Although the smaller circuits are far less prone to the higher current densities and electromigration effects, it is still beneficial to know the circuit's lifetime. Also, when looking at ways to improve design and minimize the effects of electromigration and other types of wear-out, it is the smaller circuits that need the alterations, as they are the key pieces to subcircuits and larger digital devices.

Due to limited funding for this project, I decided to use a layout tool known as Electric. The Electric ${ }^{\mathrm{TM}}$ VLSI Design System is an open-source Electronic Design Automation (EDA) system that can handle many forms of circuit design, including: Custom IC layout, Schematic Capture (digital and analog), Textual Languages such as VHDL and Verilog, and much more. This software designs MOS and bipolar integrated circuits, printed-circuit-boards (PCBs), or any 
type of circuit you choose. It has many editing styles including layout, schematics, artwork, and architectural specifications. A large set of tools is available, including design-rule-checkers (DRCs), simulators, routers, layout generators, and more. Not only does Electric interface with the most popular CAD specifications (EDIF, LEF/DEF, VHDL, etc), it provides an excellent layout-constraint system. This enables top-down design by enforcing consistency of connections and is an efficient and effective way to create and layout a large number of different circuits without having to fabricate a single one. This chapter will describe not only the choice of circuits, but the choice of layout technology.

After circuit creation and layout, I needed to find a method to generate spice netlists for each of the circuits designed. These netlists contain a ton of valuable information that the program needs to work properly. The netlist contains mosfet names, connections, nodes, parasitic capacitances and resistances, and all raw current/voltage data. Luckily for me, Electric not only has two built-in simulators (IRSIM and ALS), but can also generate decks for many other simulators (PAL, Verilog, FastHenry, Spice, and more). I am obviously only concerned with the spice deck. Spice decks can be written for all designs, and then any Spice simulator can be used to handle the netlists. I chose LTSpice, a high performance Spice III simulator, to handle design simulations and more importantly to pull the majority of information and data needed for the program.

\subsubsection{Choice of Circuit Fabrication Technology}

Electric VLSI has almost any choice of design technology available. To keep me with today's fast paced technology along with the scaling down process of digital electronics, I decided to design all of my circuits using 0.025um CMOS process technology using MOSIS (Metal Oxide Semiconductor Implementation Service) design rules. Electric represents all 
distances in dimensionless units. So, with my scale choice of $0.025 \mathrm{um}(25 \mathrm{~nm})$ process technology, a transistor that is $2 \times 3$ in size is actually $50 \times 75$ nanometers (or $0.05 \times 0.075$ microns). The MOSIS CMOS technology describes a scalable CMOS process that is fabricated by the MOSIS project of the University of Southern California.

\subsubsection{Choice of Circuits}

This project is designed to handle any catalog of circuits. Thus, I started with the layout of very simple circuits, such as AND gates, XOR gates, inverters, and simple gate chains (circuits containing a series of gates, but no more than 3 or 4 ).

Having discussed the basics of how circuit layout works, the catalog of fabricated circuits can be defined. As stated above, I started off with some very simple circuits, mostly gate level (AND, OR, XOR) and then moved on to simple gate chains, having a few logic gates tied together. I then looked at slightly bigger but still simple circuits, such as a MUX, and a full adder block. After creating a variety of smaller subcircuits, I was able to use these to generate larger more complex designs. For this thesis, I looked at an 8, 16, and 32 bit RCA (Ripple Carry Adder), a 16 bit Kogge-Stone adder, and an 8x8 register file. Below you will find descriptions of the Kogge Stone adder and the register file.

\section{CMOS Kogge-Stone}

The Kogge-Stone adder is one of the fastest single architectures for binary addition in parallel stages. It does however use a large amount of area and power The standard KoggeStone adder design for 16-bits is shown in Figure 3,2. The basic tiling block sub-circuit of the $\mathrm{K}-\mathrm{S}$ adder is a partial prefix adder cell (PPA). My design uses two types of tiles: an even tile for even rows (with row numbering starting at zero), and an odd tile for odd rows. Each tile 
compresses two prior propagate and generate signal sets into a single propagate and generate signal set for the column.

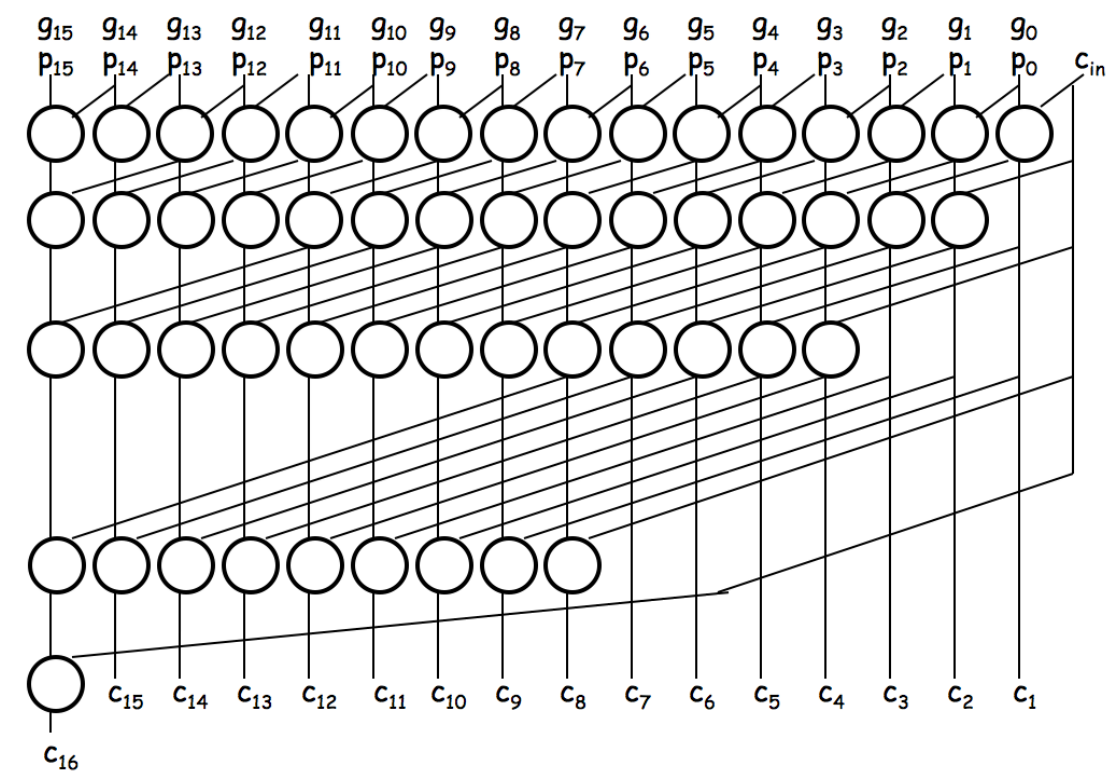

Figure 3.2: Block diagram for 16-bit Kogge-Stone prefix adder network

The critical path for the Kogge-Stone Adder is shown in Figure 3.3 consisting of 8 logic stages for the 16-bit adder. Because the CMOS logic blocks are all built with OPL (Output Prediction Logic) pre-charging transistors the timing of the entire adder is constrained to the clock.

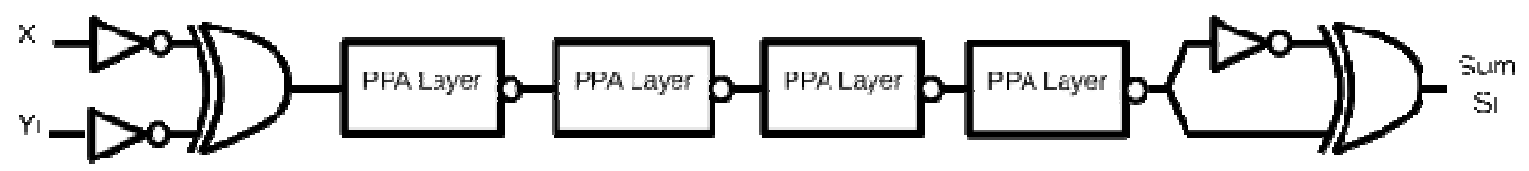

Figure 3.3: Kogge-Stone critical path of 8 logic stages for 16-bits (not all gate inputs shown)

\section{8x8 Register File}

The $8 \times 8$ register file designed for this project is fairly simple, just very large. It is comprised of eight 8-bit words, two read lines, and one write line. Each 8-bit word was formed from 8 single 12 transistor SRAM cells. 
There was nothing ground breaking about the design of the register file. Two pairs of eight MUXs are used to separate the various outputs, as it is a two-read register file, and three decoders are used to control the data flow ( 2 reads, 1 write). After all the individual words were created, all bit $0 \mathrm{~s}$ ( 8 in total) were tied to an 8:1 MUX. The same is true for all bit $1 \mathrm{~s}, 2 \mathrm{~s}$, and so on. Eight MUXES are used for the first read line, and another 8 for the second. The first eight share the same select lines, and the same goes for the second set. Three 3:8 decoders are used to control the two read and one write line operations. Thus, the eights bits outputted from the decoders are the register bits chosen to be written to or read from a given register. The circuit is not complex, it is just incredibly large.

\subsubsection{Layout Preferences}

Circuits were created incorporating parasitic resistance and capacitance. This becomes an issue when using good amounts of metal, especially when changing the interconnect sizing. Up to 6 metal layers were used in a given design, yet keeping them down to a minimum was always a design concern. As far as metal sizing, there is really no concrete way of keeping this consistent. Almost all metal lines have a width of 3 other than the power and ground rails, which are sometimes slightly wider. Silicon is set to have a width of 2 . The program assumes all metal sizes to be of width 3 unless otherwise stated in the "met" file.

\subsubsection{Creation of Spice Netlists}

Spice decks were automatically generated for each of the laid out designs, incorporating all layout preferences earlier mentioned. The spiceMultiply script was then used to create various versions of a given circuit (combination of input voltage, speed, and temperature adjustments). The netlists can then be easily simulated using LTSpice. 


\subsection{Creation of Intermediate files}

The program needs extra files in order to perform an analysis of a circuit. As stated in section 3.2, I am using a lot of tools. To get the information the other tools need, four major files are needed. They are the floorplan file, relationship file, power trace file, and lastly, the raw current and voltage data collected in Spice.

\subsubsection{Floorplan file}

One of the most important files needed for the system is the floorplan file. This file is for HotSpot. The floorplan simply divides the circuit into small "blocks" and inside each block, its contents (FETs, subcircuits, etc) are specified. For example, if one of my blocks was an inverter, I would specify the contents of the block as a PMOS and NMOS transistor. The floorplan only needs to keep track of immediate IC components, in my case transistors or subcircuits containing large numbers of them. Metal lines will be dealt with later on.

An example floorplan for a $10 \mathrm{~mm} \times 5 \mathrm{~mm}$ rectangle will look as follows:

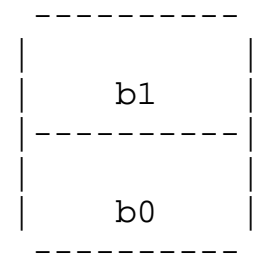

where b1 and b2 are functional blocks. The floorplan file corresponding to this example would be something like below:

$\begin{array}{lllll}\text { <unit-name> } & \text { <width }> & \text { <height }> & \langle\text { left-x }> & <\text { bottom-y> } \\ \text { b0 } & 0.010 & 0.0025 & 0 & 0 \\ \text { b1 } & 0.010 & 0.0025 & 0 & 0.0025\end{array}$

The power dissipation data, which HotSpot refers to as a power trace, would correspond to this floor plan typically as follows: 


$\begin{array}{ll}\mathrm{b} 0 & \mathrm{~b} 1 \\ 7 & 2 \\ 5 & 1 \\ 7 & 2 \\ 5 & 1 \\ \ldots & \end{array}$

where the numbers under bo and b1 are Watts per calling interval [24].

For the purposes of the project, a region of MOSFETs or subcircuits are grouped together into functional blocks and the program keeps track of the power consumption of each individual. But that information is not readily available either.

\subsubsection{Relationship File}

A file must also be made that relates each MOSFET or subcircuit or group of MOSFETs or subcircuits to a predetermined region. For this, a relationship file must be created that is specific to the program and is independent to HotSpot.

\subsubsection{Manual creation of floorplans and relationship files}

The circuits that are created for testing purposes are created in Electric, but it does not have an option to create a floor plan that is compatible with HotSpot. Floorplans were created visually in Electric; a simple Perl script was written to take the two opposite end points of a rectangular region as input and translate that to a compatible format. The input file for the Perl script also contained information on which subcircuits or MOSFETs are contained in each region. The format is as show:

scale

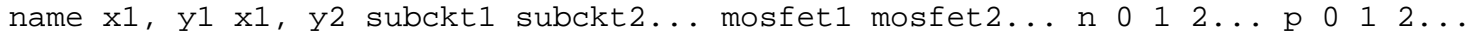

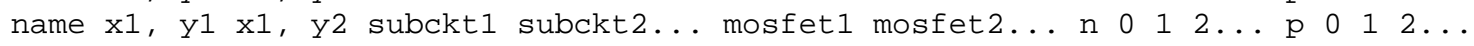
$\cdots$

The 'scale' represents the actual measurable length of each unit. For example, the distance from $\left(\mathrm{x}_{1}=0, \mathrm{y}_{1}=0\right)$ to $\left(\mathrm{x}_{2}=1, \mathrm{y}_{2}=0\right)$ would be equal to the value represented by 'scale.' The coordinate can be entered without regard to which two corners since a simple if statement can 
determine the two different cases, the case where the first coordinate represents the upper left corner and the second pair represents the lower right or if the first pair represents the lower left corner and the second pair represents the upper right corner.

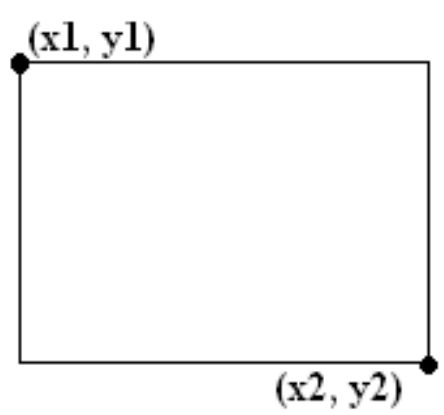

a

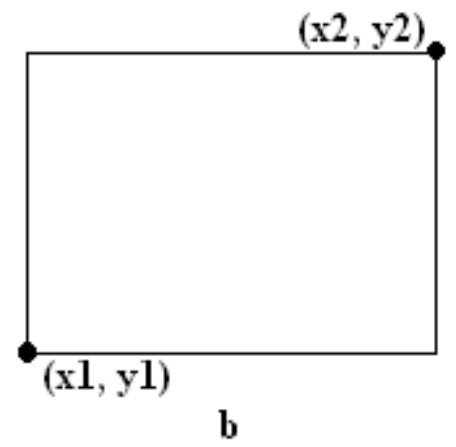

Figure 3.4: Floorplan Representation for Hotspot

Being able to have the freedom to choose where the coordinates is ideal because in Electric, the toggle measurement tool can show multiple continuous measurements as shown in figure 3.5. As can be seen in a ring oscillator, several inverters are grouped together. The colored rectangular boxes represent which subcircuits are grouped together.

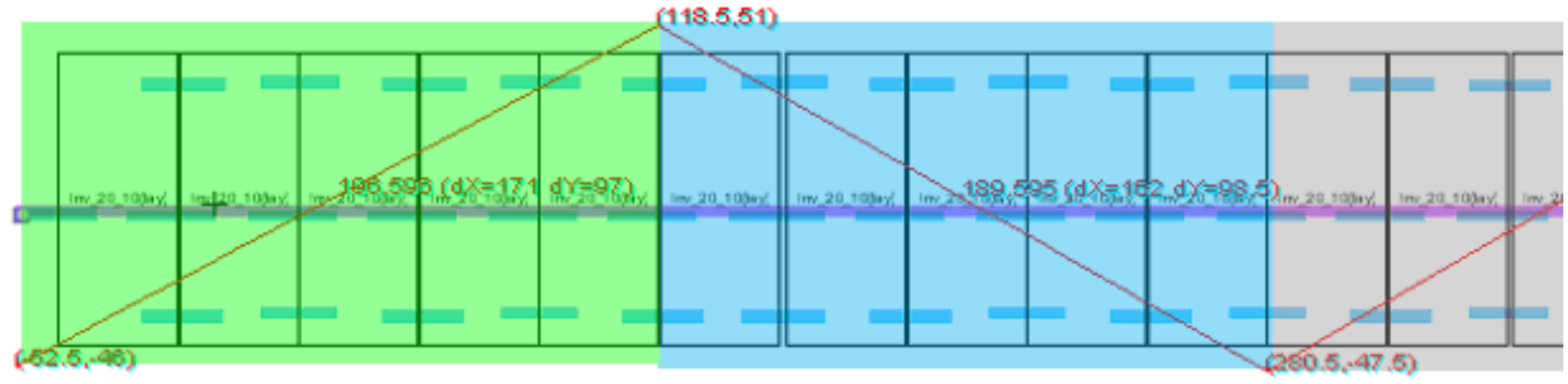

Figure 3.5: Use of Toggle tool to create floorplan files

The parameters after the coordinates are for the relationship file. The name that Electric gives each subcircuit and MOSFET instantiation, follows the coordinates. The ' $n$ ' and 'p' statements 
are used as a shortcut since Electric provides a default MOSFET name of “mnmos@0” or mpmos@0.” The numbers following the 'n' and the 'p' will generate the appropriate name.

The floor planning script will read each line and create two files: the floorplan which HotSpot needs, and the relationship, which the program needs.

\subsubsection{Power Trace File}

The power trace file is created in the main program using the LTSpice data. This file is created by the program. HotSpot needs the power trace file to run a temperature analysis for each functional block defined in the floorplans. In order to create the power trace file, the program needs both the relationship file and the raw current and voltage data from LTSpice. With the data file, the program calculates the power dissipated by each MOSFET for every half period as defined in the transient response. Once each power value is calculated and saved, the power dissipated from MOSFET in each region is added together and outputted to a power trace file that is compatible with HotSpot.

\subsubsection{Raw current/voltage data from spice}

The final file needed for the program to run is the raw current and voltage data outputted from LTSPice. This is done by running a simulation in LTSpice given a spice file with inputs and a transient analysis statement and exporting the data. This will produce a file with the currents output from every voltage or current source and the currents that flow in and out of the various terminal of each MOSFET and the voltages of every node. Ideally the inputs are to stress the circuit as it would be stressed in real life as the program assumes that the inputs are repeated for all time. For example, if an inverter was tested with a square wave input that switches every 
$1 \mathrm{~ns}$, the program will determine a mean time to failure assuming that the input stays the same for all time. So the inputs have to be chosen intelligently in order to get an accurate MTTF calculation. The only problem with this approach is the amount of processing time and memory needed to produce the files. For slow computers simulations of large circuits could take a long time and the files can be well over 100megabytes.

\subsection{Software Tools/The Program}

The program is written in $\mathrm{C}$ in a Unix environment because of my familiarity with the programming language and also because it can execute other Unix based programs which makes running the program for the user much simpler. The program is divided into 6 major functions: processConfig () - this function processes the configuration file for each simulation of the circuit. This file is crucial to the program as it provides the details of all the important paths and files needed for the program to run and other necessary information.

create () - this function reads the pspice file and catalogs all MOSFET and connections.

assignRegions () - once each MOSFET is cataloged the relationship file is read and assigns the regions to each individual MOSFET.

monitor () - this function monitors the voltage at the gate of each mosfet and then current in each connection.

printPower () - this function creates the power trace file. 
get Temperature ( ) - this function calls HotSpot and reads its output(temperature) and assigns the appropriate temperature to the appropriate MOSFET.

changemetal () - this function reads in a file and changes the line widths between transistors to the widths specified in the file. The metal heights are assumed to be the same throughout the circuit.

$\operatorname{dMTTF}()$ - given an average gate voltage and a steady state temperature, a mean time to failure is calculated.

When the program runs its course it will output an excel file with the name of each MOSFET as named by LTSpice, a mean time to failure, a temperature in Kelvin, an average electric field, a gate width, the total power dissipated by the MOSFET, and for the whole transient analysis.

\subsubsection{Extracting Runtime Information}

The program needs multiple pieces of information to run and the configuration file that it reads in provides that information. The information includes the location of the HotSpot program, the name of the circuit file to test, the name of the relationship, floorplan, raw data, and the metal width file. The other information it extracts is used to modify the MTTF equation: A* $308.3401+0.9207 *\left(100-\mathrm{T}_{\mathrm{s}}\right) * \mathrm{e}^{\wedge}\left(\mathrm{B} *\left(-1.089 \mathrm{E}-06+8.9344 \mathrm{E}-09 *\left(100-\mathrm{T}_{\mathrm{s}}\right)\right) * \mathrm{~J}\right)+\mathrm{C}$. Three constants can be set that represent the constant in front of the equation, the multiplier inside the exponential term and an offset. The rest of the data is information used to produce the excel spreadsheet that has the MTTF data. 


\subsubsection{Creating a catalog}

In order to catalog each MOSFET, the program needs to read the PSpice file. It goes through the file line by line looking at the first word of each line. Resistors and capacitors are ignored but subcircuit definition, MOSFET and subcircuit declarations, and the .TRAN statement are each treated in a special way.

When the program reads in a MOSFET, it creates a MOSFET object recording its name and whether it is a PMOS or NMOS FET. Three connection objects are created representing the metal line between the gate, drain, or source to a node. The objects are "attached" to the MOSFET terminals. Next the nodes that the gate, drain, and source are connected to are checked to see whether or not those nodes are already created by previously MOSFETs. If the node or nodes exists then the connections object are connected to the appropriate nodes. If the node does not exist, the node is created and named as declared in the PSpice file. So with each line with a MOSFET declaration, a MOSFET is created and is connected to three nodes.

When the program reads a subcircuit definition, the program behaves as if it is creating a separate circuit. It first creates the appropriate number of interface nodes or outer nodes and then as it reads a MOSFET line it creates the MOSFETs and the nodes if necessary. The names of each MOSFET and node reflect that it is part of a subcircuit. The resulting circuit is saved into a catalog.

If a subcircuit declaration is encountered, all the MOSFETs and nodes are copied and if a declaration was encountered inside a subcircuit then the MOSFETs and nodes are renamed to reflect the fact that they are inside a subcircuit and connected with the rest of the main circuit. 
Luckily, Electric creates a spice file with the inner most subcircuits appearing closer to the top of the file before the subcircuit that contain further subcircuits appear.

When the program encounters the .TRAN statement, the first argument is saved and is interpreted as half the period of the fastest input. This is because the monitor() function will record several power readings and each individual power reading will be the power over that interval. The second argument is saved as the entire length of the transient analysis. Ultimately all the nodes and MOSFETs are saved into a circuit object which makes accessing each individual circuit element easier.

The main job of the create() function is to record the names of each node and MOSFET as LTSpice knows it as because the LTSpice output file contains the specific names and if the names aren't recorded exactly, then the various currents and voltages cannot be monitored.

\subsubsection{Assigning MOSFETs to regions}

Once the MOSFETs are named appropriately, they must be assigned to a region. In order to do this the relationship file is read in. The format for this file is:

REGION mosfetName mosfetName ... subcircuitName subcircuitName ...

In order to assign the regions, the words after the 'REGION' are compared with the name of each MOSFET in the circuit object. If a match is found the 'REGION' is saved into the MOSFET. If there is an error in the relationship file, for example if a MOSFET is not assigned a region, the program stops execution. A list of the regions is also saved into the circuit object in order to quickly access the information for creating the power trace file. 
Because of the complexities of the metal lines that connect each MOSFET, the metal lines that connect the MOSFET to each node are assumed to be inside the region of the MOSFET even though they may not be in Electric.

\subsubsection{Monitoring of circuit usage}

After everything has been cataloged, monitoring can be done via processing the LTSpice data file. The top line of the data file contains the traces so the first line is processed element by element; for example, an element may be Id(MOSFET) or V(node) and the information in the same column represents the value at a certain time. The point in time is the first element displayed on each line. If the name inside the parenthesis matches the name of one of the MOSFETs or nodes, then its position or index is saved in the node or connection object. This is done to quickly access information within the file. The indices that are saved are nodes connected to the gate, drain, and source, and the Id, Ig, and Is parameters which represent the drain, gate, and source current from the MOSFET. If a node or connection is not defined by an index number, the value is assumed to be zero for all time.

After the indices are saved the file is processed line by line. Power, voltage, and current averages are calculated using a left Riemann Sum. This is done because the information provided by the data file is expressed like in figure 3.6. At a certain specific point in time the voltage or current can be a certain value. Because it would be too difficult to get the remaining points and interpolate the data in an equation so the left Riemann Sum is a lot simpler to program. Great error is avoided however because each time step is not set, LTSpice makes the time steps as small or as big as necessary with large steps having data that is mostly constant. The average 
calculated in the program has been compared to the average calculated by LTSpice and the value is equivalent.

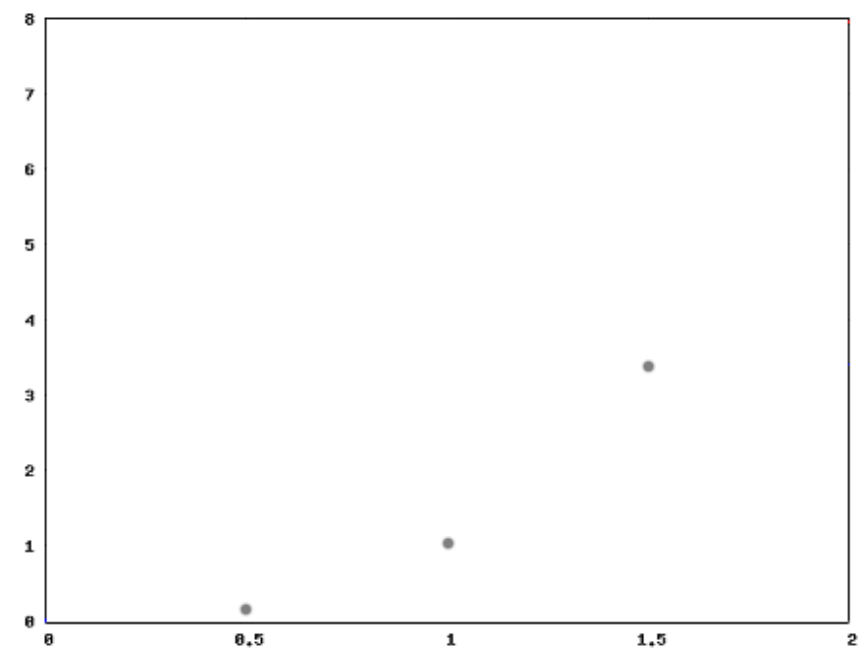

Figure 3.6: Data as it appears in the file

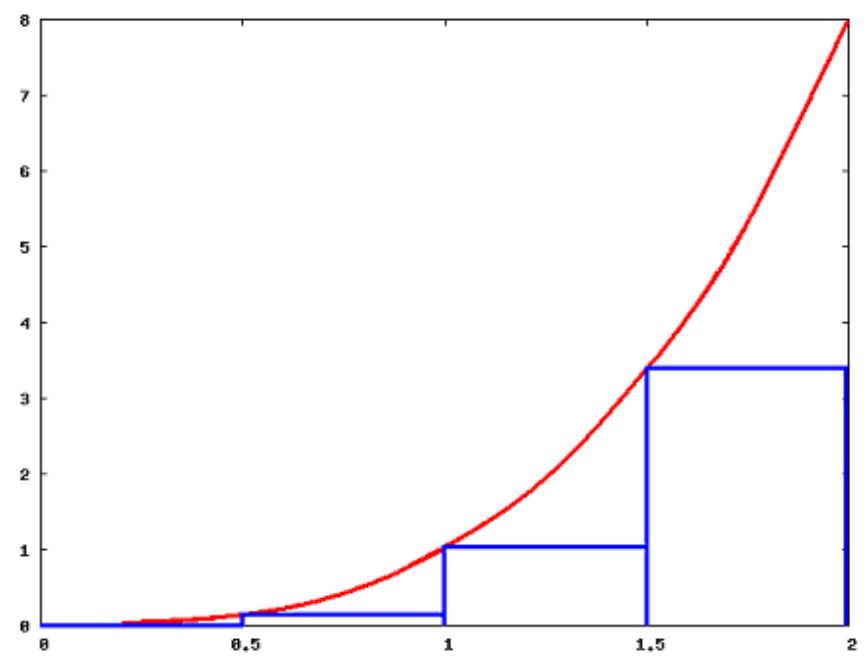

Figure 3.7: The left Riemann sum of the data

So order to calculate the average power in an interval the program goes through each MOSFET and gets the voltage at the drain, source, and gate as well as the currents into and out of the MOSFET and calculates the instantaneous power and multiplies that by the difference between the current time and the time at the next iteration. Once the time goes past an iteration of half of the period of the fastest input, the average power is saved into the MOSFET object. So if the 
fastest signal switches every $1 \mathrm{~ns}$ and the transient analysis is 10ns long, each MOSFET object will have 10 power values each representing the average power in each interval.

The average gate voltage and the average current of each connection is calculated the exact same way except that the value is calculated as an average for the entire transient analysis instead of for each half period. Once the end of the data file is reached, monitoring is complete and the function returns to main.

\subsubsection{Creation of the Power Trace File}

When the power has been calculated, the power trace file can be created. The program goes through the list of regions saved in the circuit object and again the program uses a linear search to find out which MOSFETs are in the region being searched for. The average power for the interval for every MOSFET "in" the region are added together and ultimately outputted to the

power trace file. This procedure is done for every region and every interval, one hundred times in order to give HotSpot the data necessary to determine a steady state temperature.

\subsubsection{Calling HotSpot}

Once the power trace file is created, the program executes HotSpot and provides the appropriate arguments that would allow the temperature in each region to be calculated. The output of HotSpot is redirected to the program and the data is parsed and the temperature is saved to each individual MOSFET in a particular region.

\subsubsection{Changing Metal Widths}

For cases when metal lines need to be adjusted from their default widths, the program reads in a file and adjusts the appropriate lines. Because of the limitations of the program and the lack of geographical and dimensional information from the layout tool, the connection between the 
various terminals of the MOSFET are assumed to be straight lines that do not turn and have the same dimensions even though they may not.

\subsubsection{Calculating MTTF}

After all the important information has been calculated and saved, a mean time to failure for each MOSFET can be calculated. The equation created from the work mentioned in section 2.5 is used with the inputs being temperature and voltage values. As mentioned in 3.5, the program outputs a spreadsheet file with additional information, which is done in the MTTF() function. The total power for each MOSFET for the entire transient analysis is also calculated.

\subsection{Addition Tools}

C's primary function is not to be a text processor, for that Perl scripts were written to set up the test environment and also to calculate extra pieces of information that could be important when trying to find a correlation between anything else and mean time to failure.

\subsubsection{Setting up Multiple Tests}

One script was written to create spice files, configuration files, and shell scripts that would test a circuit under various conditions. More specifically, the spiceMultiply script takes in a single Pspice file, a configuration for the program, and a parameters list, and creates multiple copies of the spice file under different conditions and shell scripts to simulate the circuit and calculate its MTTF.

The parameters list allows the user to vary the conditions under which the circuit is simulated; the input speed, the temperature at which the circuit is run and the rail voltage can be changed. Also multiple constants can be entered which will modify the MTTF equations. The script will produce a circuit file for every combination of speed, temperature, and VDD. It will also create a 
shell script to run the appropriate pre and post scripts and simulate each version of the circuit and calculates its MTTF. As each of the individual files are created, a larger shell script file is created that will invoke each of the individual shell scripts effectively running the entire simulation and take the lowest MTTF for EM and write its information to an excel file.

\subsubsection{Changing the Temperature}

HotSpot can calculate temperature at a different started temperature as the temperature at which the PSpice file is simulated under. In order to avoid this and ultimately producing erroneous results, a script was created to consolidate, the two files. The script reads the argument of the .TEMP parameter in the PSpice file and alters the initial temperature in configuration file of HotSpot.

\subsubsection{Converting the LTSpice Utility data file}

The format of the raw data file that the program can process is different than that which LTSpice and the LTSpice Utility produces. In order to make the file compatible a script was written to convert those files to a compatible format.

\subsubsection{Calculating Average Power for each Region}

The other important information that could be used is the average power of each region. This is done by simply adding up all the values for each region in the power trace file and dividing by the number of iterations. The average power is appended to the end of the spreadsheet created by the program. 


\subsubsection{The Speed Tester}

One script was written to test the delay for a circuit given the data file and the rail voltage. This can be done manually by looking at the when a signal reaches $10 \%$ or $90 \%$ of its maximum depending on if the signal should be high or low. This is too tedious and not efficient especially if, a circuit has multiple outputs and the transient analysis is very long compared to the period of the fastest input. For example, trying to find the delay for every possible output of a 4 bit ripplecarry adder would require looking at 5 different signals 512 times.

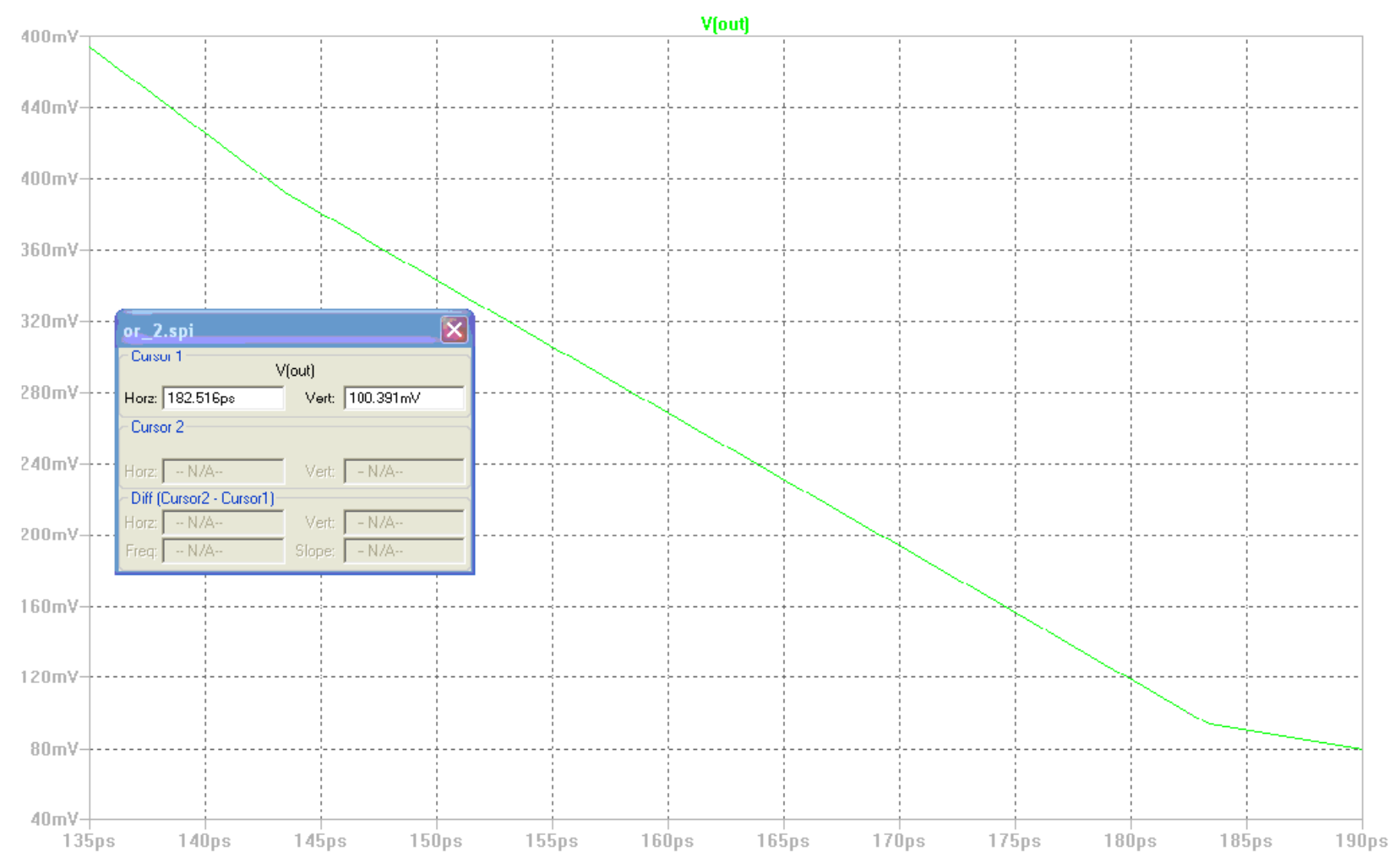

Figure 3.8: Showing method for manually finding delay

The alternative is to use the data file again to determine delay. So a script was written. The script needs three pieces of information, the nodes to monitor, the rail voltage, and the value of half the period of the fastest input. As was done with the monitoring section of the program, given the nodes to watch, an index can be assigned to the location of the particular node. With the rail 
voltage the script can calculate the $10 / 90 \%$ values. And finally the half period value is needed to tell the script when to start timing the delay.

Assuming a circuit is working properly, or it is not driven faster than it can function, the script can determine if that particular iteration of the output should be a high or a low. This is done by checking the value of the node right before the start of the next iteration. That is if an inverter has an input signal that switches every $1 \mathrm{~ns}$, in order to tell if the output should be high or low, the script checks the value of the output node at 0.999 ns since the output will not switch before the input is changed.

Once that information if acquired the next thing to do is measure the delay. Because LTSpice does not usually have a value at exactly the $10 / 90 \%$ point, the script gets the two points around the 10/90\% point and calculates the equation for the line connecting those two points as a function of voltage. Once that is done the script plugs in either the $10 \%$ or $90 \%$ value to get a delay estimate. As seen in figure 3.9, this can create some error because the signal will not be linear, but this is as accurate as measuring the delay by hand because as seen in figure 3.10, LTSpice has line segments connecting the points. 


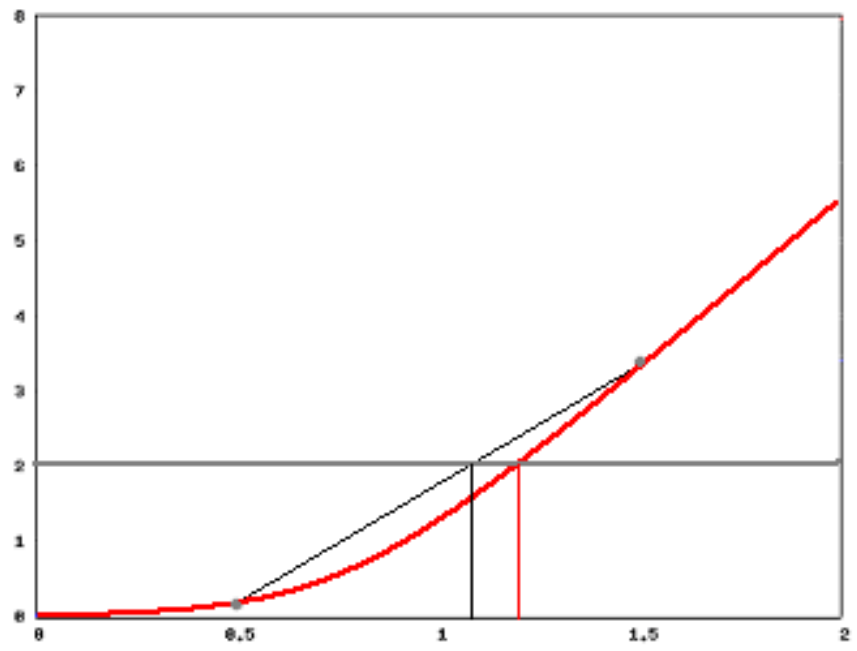

Figure 3.9: Error possibility in Delay calculations

Another potential problem is glitches. This is accounted for by keeping track of which nodes cross the $10 / 90 \%$ value twice in one interval. With that information the first crossing can be ignored and the data from the second crossing can be used to calculate delay.

The script calculates the delay for each iteration for every node and keeps track of the largest delay and appends that value to the end of the spreadsheet created by the program.

\subsubsection{Gathering the Results}

If a spice file is created for every combination of three temperatures, three input speeds, and three rail voltages, 27 different analyses would be done by the entire tool chain. In order to combine all of the results, a script was written. The script goes through every excel spreadsheet that was created, locates the one with the lowest MTTF and copies its information to a larger excel spreadsheet. The final result is a spread sheet organized by the different constants, temperature, rail voltage, and input speed having the data of all 27 analyses. 
A sensitivity analysis was done as well. But this was done through an excel spreadsheet. The equations from the models were simply put into the spreadsheet and the effects of the current density or temperature were amplified. That data will prove useful when analyzing a circuit where the effects of temperature are too great to counteract any sizeable reduction in current density. The data added to the spread sheet was the MTTF when the circuit does not add any heat to the ambient temperature, the MTTF when current density is at a value that maximizes the life of the circuit, and the MTTF for when the effect of the temperature is increased. 


\section{Chapter 4. Circuit Evaluation}

This chapter deals with the actual model usage for determining the failure times of various circuits. I have created a catalog of circuits to test, some very simple and others quite complex. I will now evaluate the circuits and discover just how and when they fail. I have a method for monitoring temperature and current density and now the equation can do the rest, verifying that the model created is in fact accurate. All circuits described in Chapter 3 were simulated at a variety of temperatures. High temperatures were used as the ICs used in industry operate at such conditions [28]. Hotspot is keeping record of temperature for the various designs, and if discrepancies are found between HotSpot and the temperatures specified in the spice files, designs were adjusted and simulations were once again performed.

\subsection{Testing the Circuits}

The circuits were tested under various conditions. By default, metal lines have a thickness of 3 units, or $75 \mathrm{~nm}$. Three different variables were changed, the rail voltage, and consequently the input voltage, the starting ambient temperature of the circuit, and the switching frequency of the inputs. The rail voltages chosen were $1 \mathrm{v}, 1.25 \mathrm{v}$ and $1.5 \mathrm{v}$. The starting ambient temperatures chosen were 100 degrees Celsius (373 Kelvin), 150 degrees Celsius, and 200 degrees Celsius. The input frequencies chosen to simulate a circuit are $400 \mathrm{MHz}, 100 \mathrm{MHz}$, and $25 \mathrm{MHz}$. Originally $25 \mathrm{MHz}, 50 \mathrm{MHz}, 100 \mathrm{MHz}, 200 \mathrm{MHz}$, and $400 \mathrm{MHz}$ were chosen, but having 5 different values for switching frequency would have increased simulation time by over 50\%. For most cases, such as with the gate and the smaller circuits, the inputs were selected so as to get every possible input output pair effectively testing out an entire circuit, but for larger circuits, a certain input was chosen to test a certain part of a circuit. 
All the data for each of the cases were collaborated and the date for the "weakest link" or the MOSFET that had the lowest MTTF was collected and placed into a single spread sheet. Using this spreadsheet, a variety of graphs was created.

The circuits were tested in an environment where connections between terminals of a MOSFET are wires which have both parasitic capacitance and resistance. This adds to the delay of a circuit as well as acts as a load that each gate or subcircuit must drive. More power is consumed and temperatures become higher, yielding a lower MTTF. Simulating with parasitics in mind is important, as any changes to increase overall lifetime may change parasitics in ways that increase power and disperse more heat. For example, reducing interconnect sizing can increase MTTF but also increases parasitic resistance causing an increase in power consumption and temperature.

\subsection{Verifying the Models}

To verify the model created, a ramp test was performed on a single inverter similar to that done is various papers. The input of the inverter was changed from $1 \mathrm{~V}$ to $1.5 \mathrm{~V}$ in increments of $0.25 \mathrm{~V}$ at three different temperatures, 373,423 , and 473 Kelvin. The program was run and the MTTF data points were compared to the original paper's data. The results from the constructed tool chain pretty closely match the data found in papers [25] and [26]. The papers looked at a single FET, so there are subtle differences. There is a slight bit of error, but this comes as a result of manually recreating the paper's data in excel. Also, the equation must be more generalized to handle all current densities and temperatures. For purposes of this paper, all graphs showing MTTF relationships were normalized to the lowest value, and show the percent difference between that value and all other values in the set. 


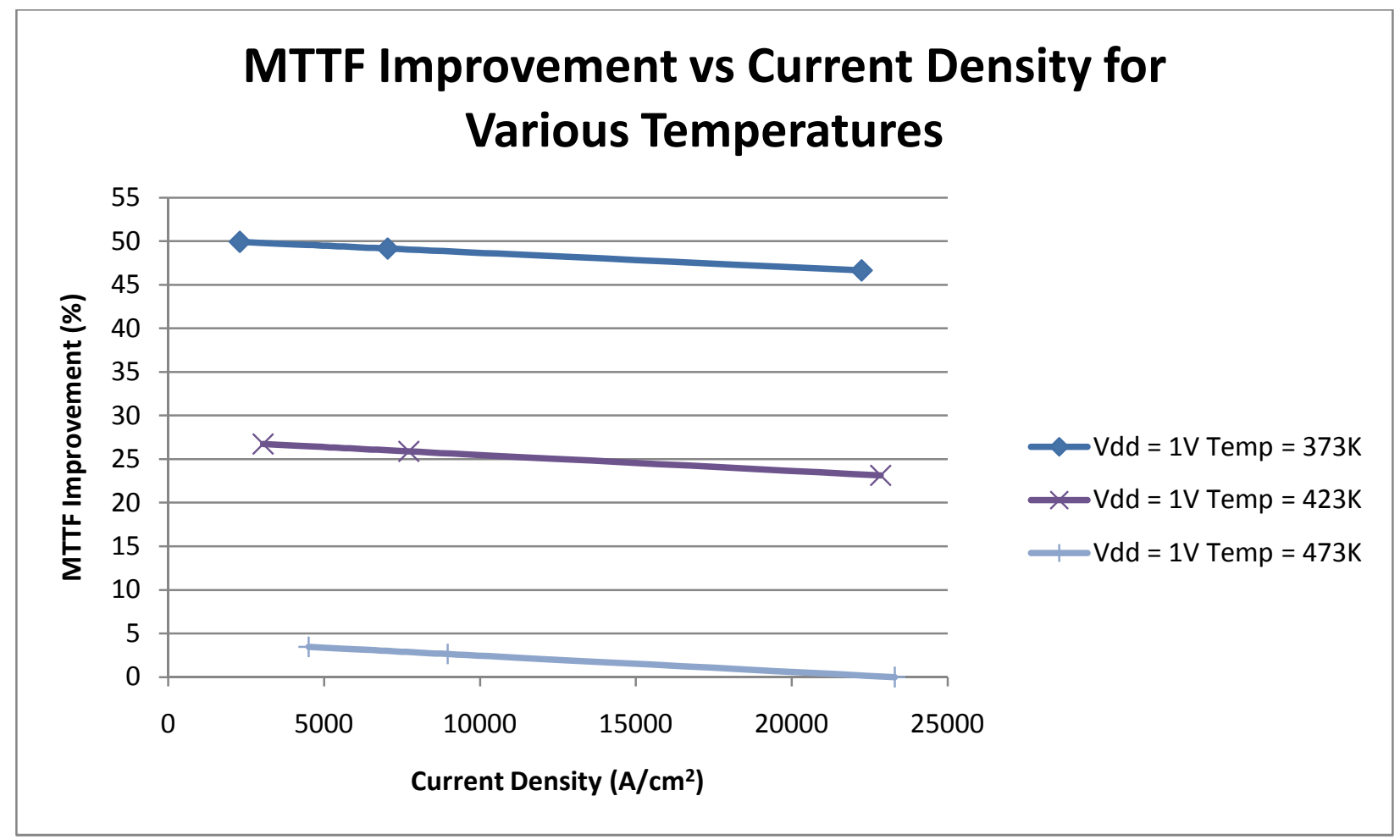

Figure 4.1: MTTF Improvement vs. Current Density Plot Using Created Model

\subsection{The Inverter}

The inverter is the most basic gate in a digital system. Using the findings for an inverter, I can get a general idea of wear-out behavior for larger circuits and use the tool chain to estimate failures. Also improvements can be made to larger circuits in order to increase lifetime with minimal to no decrease in performance. In order to study the relationship of various factors against mean time to failure, inverters were simulated at 3 different starting ambient temperatures and 3 different rail voltages. Widths of metals at source and drain locations ranged from 6 units to 27 units ( $150 \mathrm{~nm}$ to $675 \mathrm{~nm}$ ). The input to the inverter was a square wave of varying frequency with a $50 \%$ duty cycle. 


\subsubsection{The effects of Input and Rail Voltage}

With an increase in input/rail voltage comes an increase in current. Because this means more current through the same size interconnect, a higher current density is expected. Thus, with input voltage being proportional to current density, an increase input voltage yields a reduced MTTF. This was seen in section 4.2. However, because current densities for the inverter are so small $\left(<10^{6}\right)$, the increased current densities as a result of increased input voltage yield only a very small change in MTTF. When the rail voltage is increased however, the delay decreases, giving a performance increase. The data in figures 4.2 and 4.3 show the effects of increasing the rail voltage.

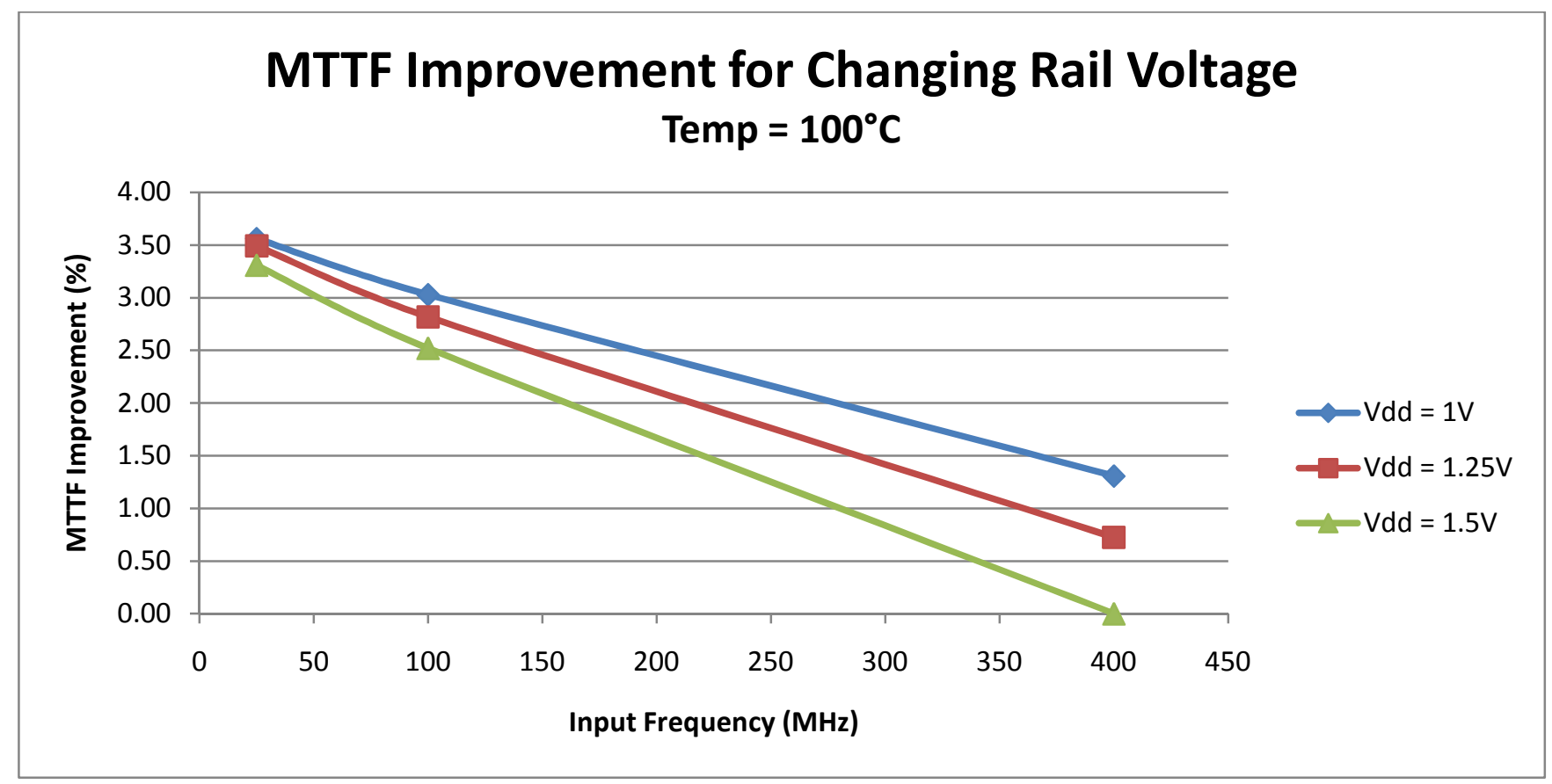

Figure 4.2: Effects of Increased Rail Voltage on MTTF 


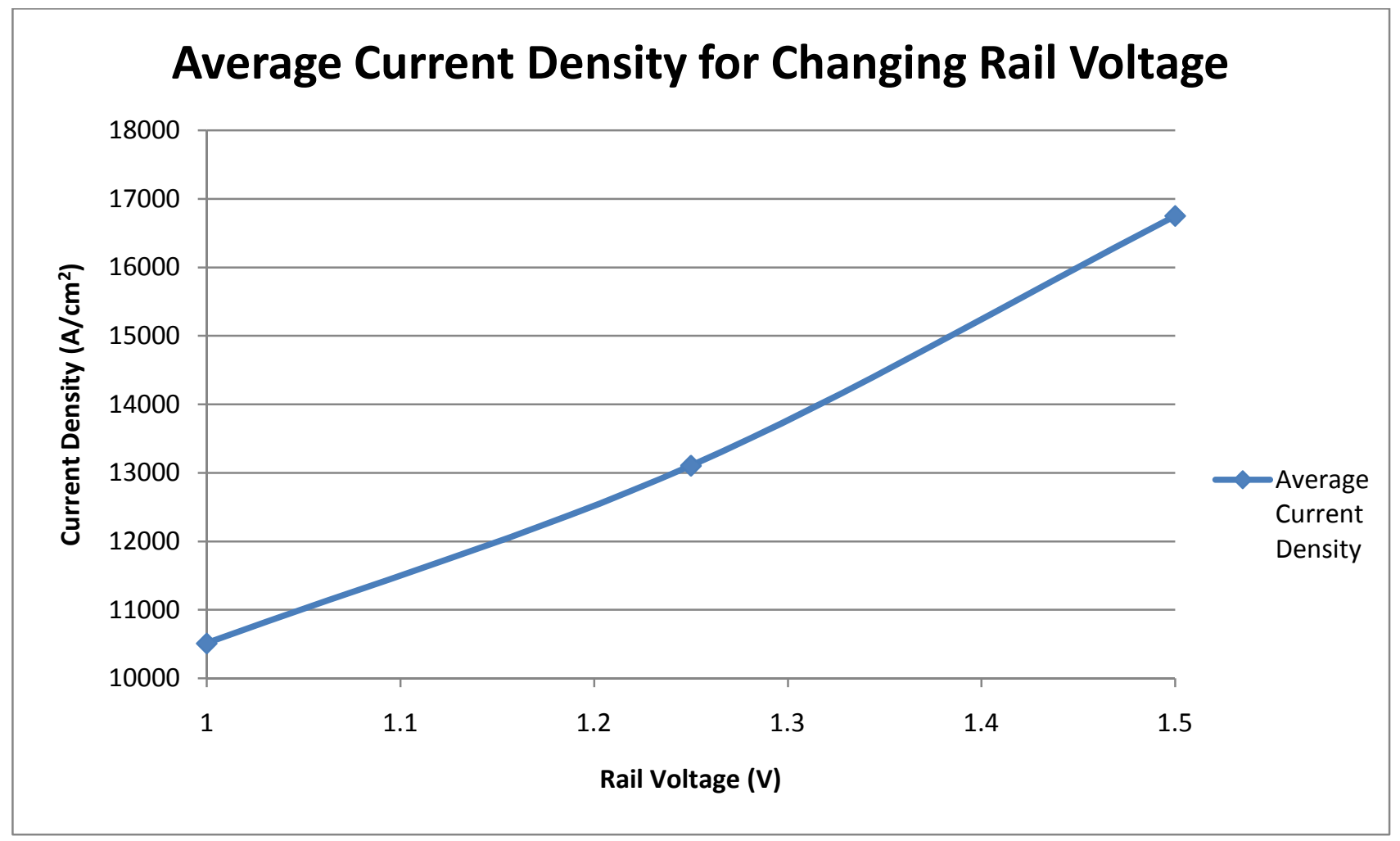

Figure 4.3: Effects of Increased Rail Voltage on Current Density

As you can see from figure 4.4, MTTF does in fact change with a change in input voltage, but the changes are minimal. It is safe to assume then that at lower current densities (found in the small circuits/basic gates), ambient temperature of the device has a much greater effect on MTTF. I can also see from the above figure that as current densities become greater, they have a larger effect on MTTF of the device.

\subsubsection{The effects of Temperature}

Temperature is the second biggest factor affecting MTTF other than current density. For very small circuits, temperature outweighs the effects of current density. However, this paper focuses on larger circuits. With temperature also comes an increase in current density, but the increase in not nearly as much as that with an increased input voltage. However, because I am running these 
circuits at high temperatures by default, any slight increase in ambient temperature of the device is sure to decrease its lifetime.

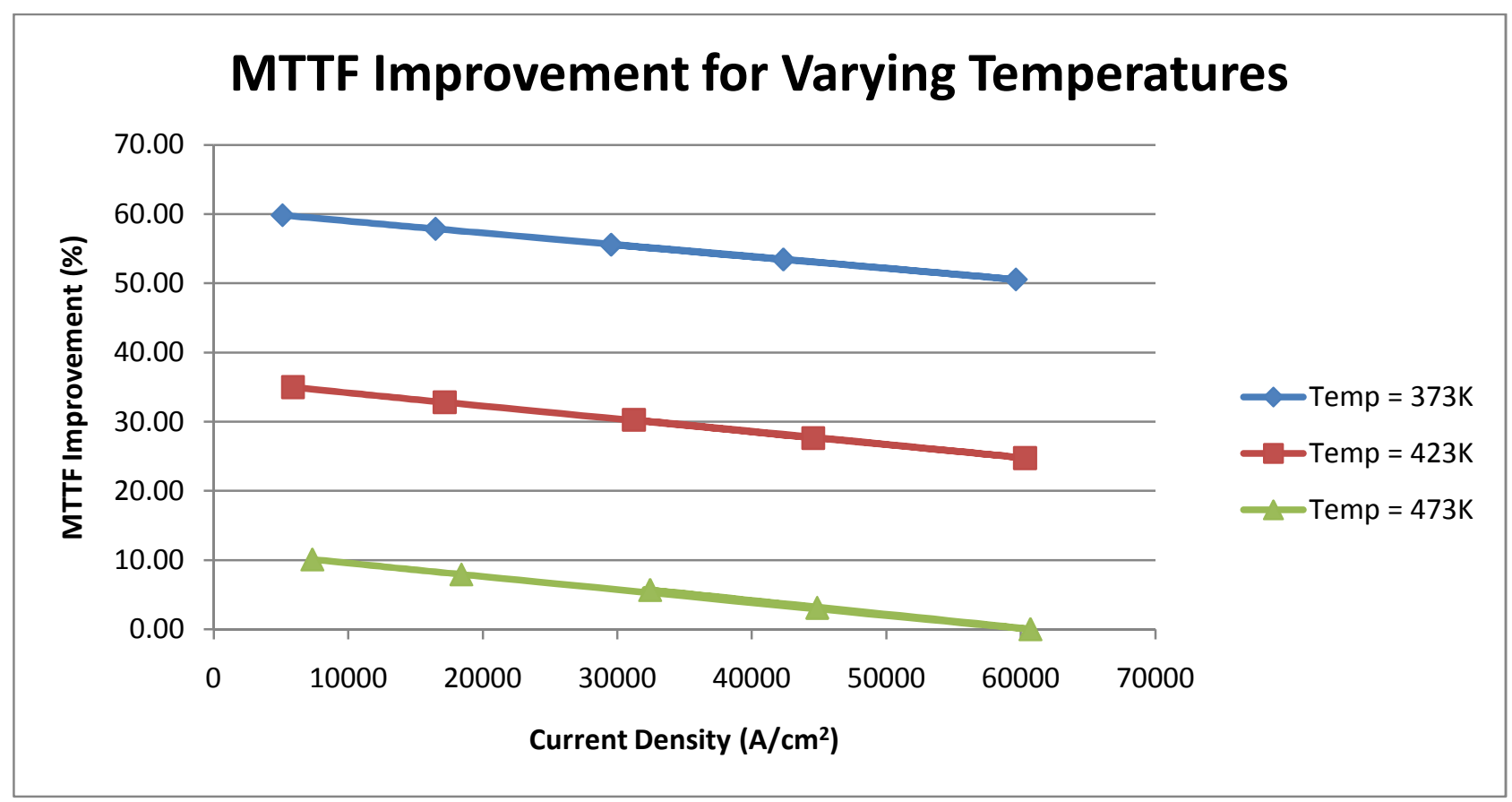

Figure 4.4: Effects of Temperature on MTTF for Inverter

As can be seen in figure 4.4, the relationship between current density and MTTF for the inverter is fairly linear. This is probably due to the lower current densities associated with the small inverter. However, the change in MTTF with change in temperature is quite large. I only ran my circuits to 473K (200 Degrees Celsius), however, it can be easily seen that at extremely high temperatures, the circuits will indeed fail very quickly. As the circuit is dissipating more heat, it in turn will use more power and its lifetime decreases. The amount of power the inverter dissipates greatly affects the lifetime of the inverter. This is because the power used by the inverter creates heat, heat that HotSpot calculates based on power data. Obviously a smaller circuit that uses a certain amount of power will become hotter than a larger circuit that uses the same amount of power. As can be seen in figure 4.5, the power usage is directly proportional to 
the MTTF of a circuit regardless of the size and configuration of the inverter. The data shown is the MTTF plots for five different inverters. Each point represents the power usage of the inverter using a different metal interconnect size for source and drain metals.

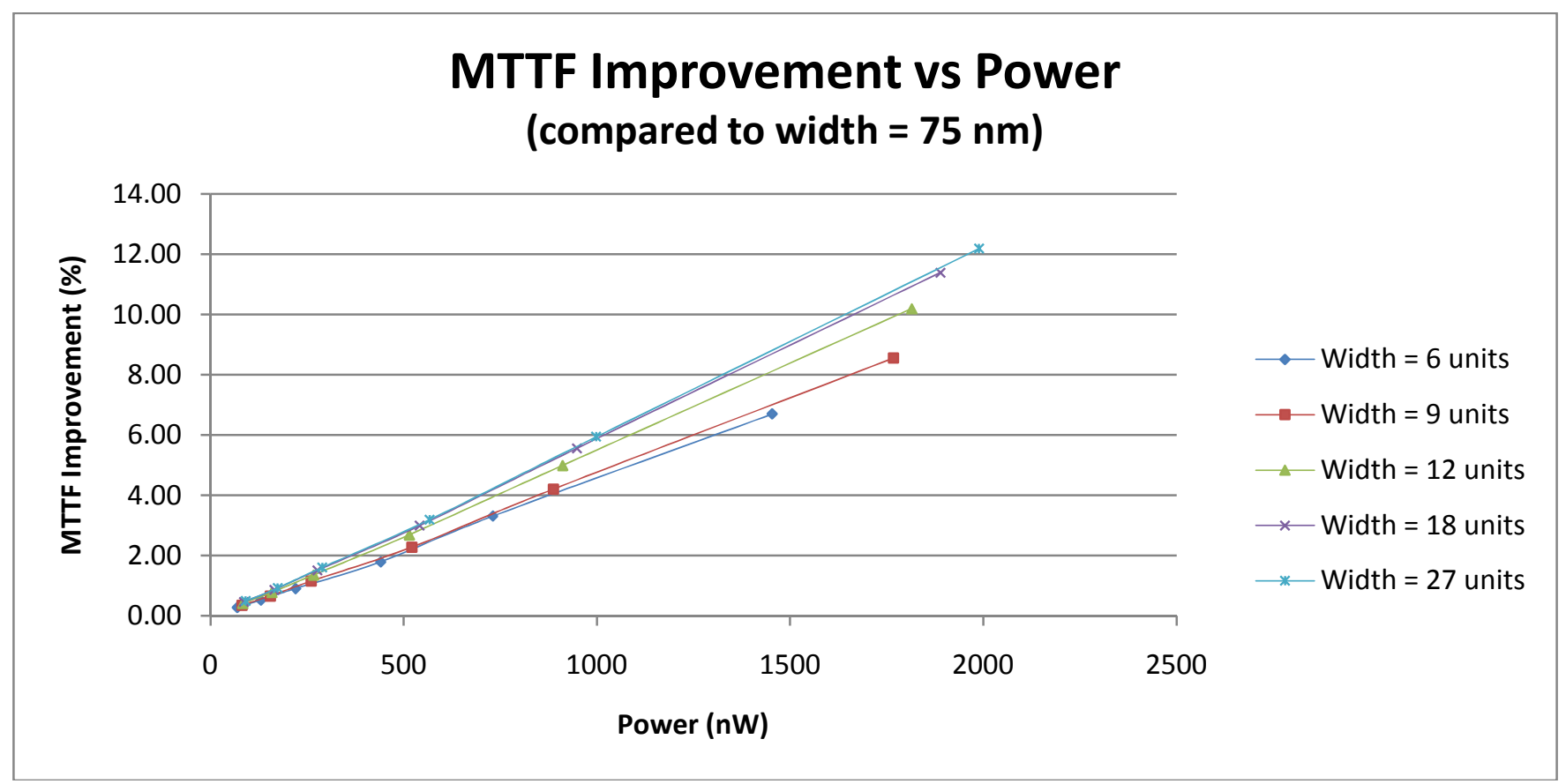

Figure 4.5: Power vs. MTTF plot for Inverter

The amount of power a CMOS circuit consumes is proportional to the frequency times the load it is driving times the square of the rail voltage as seen in equation 4.1. The above tests (other than the input voltage section) assume a constant input of $1 \mathrm{~V}$ with a variation in interconnect width for source and drain metal lines. Clearly, the more power the inverter consumes, the faster it will fail. On the larger circuits to be discussed in later chapters, changing interconnect size will have strong affect on the MTTF, because again I will be dealing with much larger, higher current density devices, all that consume more power.

$$
P=\frac{1}{2} f_{C} C_{L} V_{D D}^{2}
$$

\section{Equation 4.1}




\subsubsection{The effects of Input Frequency}

Many high speed electronics consume large amounts of power. With CMOS technology, most of the power consumption is due to the MOSFETS transitioning through the linear region to either saturation or off. Because I am talking about an inverter, no matter whether the input is a logic 1 or 0, either the PMOS or NMOS will be off. No current flows into the gate terminal, and there is no dc current path from Vdd to GND, yielding a steady-state current of zero. This means zero static power. Sometimes I will get little amounts of static power due to reverse-bias leakage between diffused regions and the substrate, but for my purposes, I will ignore static power. The majority of power consumed is dynamic and is caused by switching logic states. Dynamic supply current is dominant in CMOS circuits because most of the power is consumed in moving charges in the parasitic capacitor in the CMOS gates. As mentioned in section 4.3.2 power contributes to the thermal effects that can lower the MTTF. Figure 4.6 shows the power consumption of the inverter for input frequencies of $25 \mathrm{MHz}$ to $800 \mathrm{MHz}$.

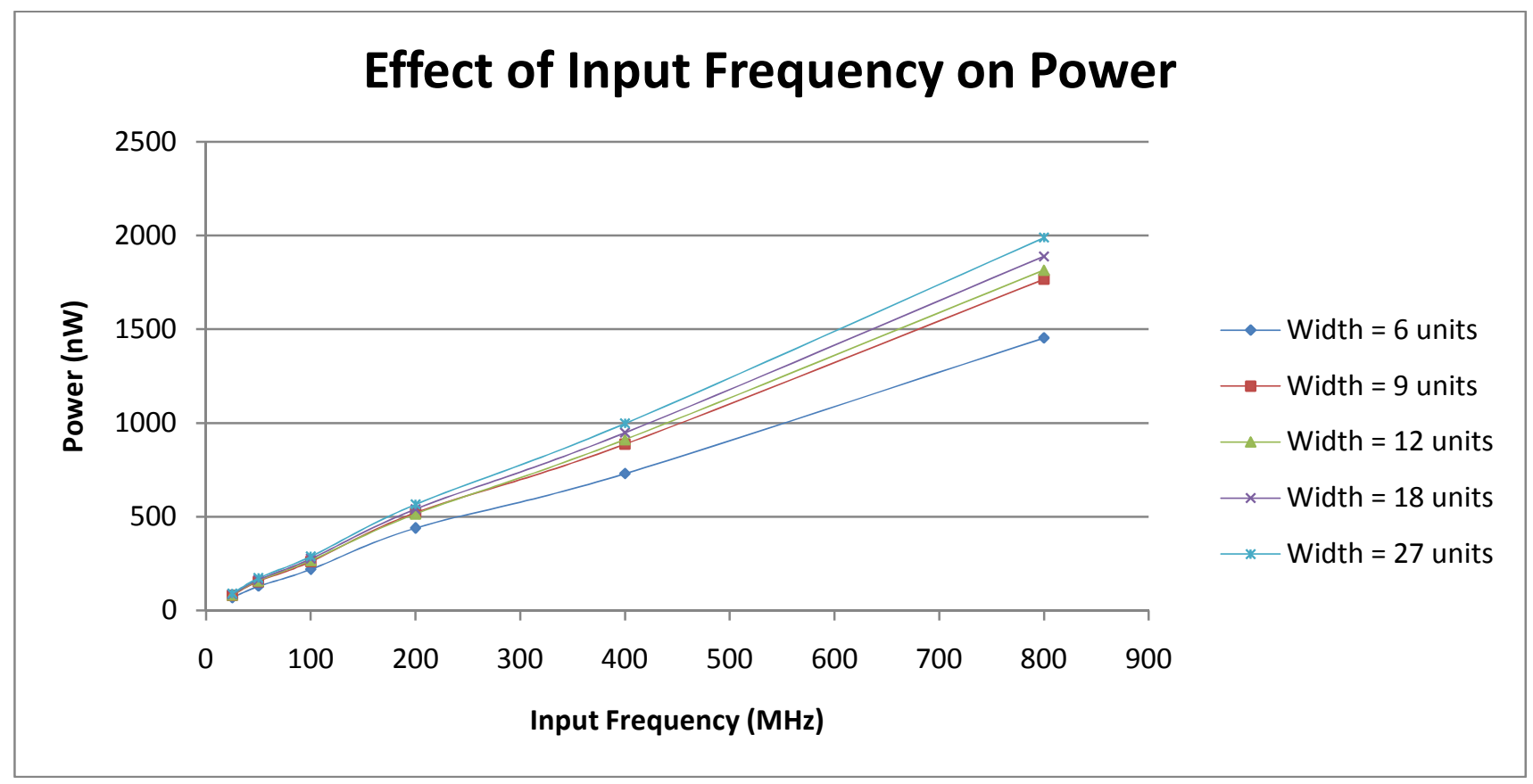

Figure 4.6: Effects of Input Frequency on Power for Inverter 
So the faster the input frequency, the more average power the inverter consumes, which yields a higher temperature and thus a lower MTTF. Figure 4.7 shows the relationship between input frequency and MTTF for different interconnect sizes. As expected, the inverter with the largest interconnect size dissipated the most power, which in turn increases temperature. This leads to a lower MTTF. However, the increase in interconnect size reduces current density, which should increase my MTTF. Again, because I am dealing with smaller circuits with lower current densities, the power consumption and temperature increase greatly outweigh the reduction in current density. At very low input frequencies the MTTF is less affected by switching frequency, and more by changes in temperature and current density. Yet, at much higher frequencies, the switching frequencies tend to take over the increased temperature and current density reductions.

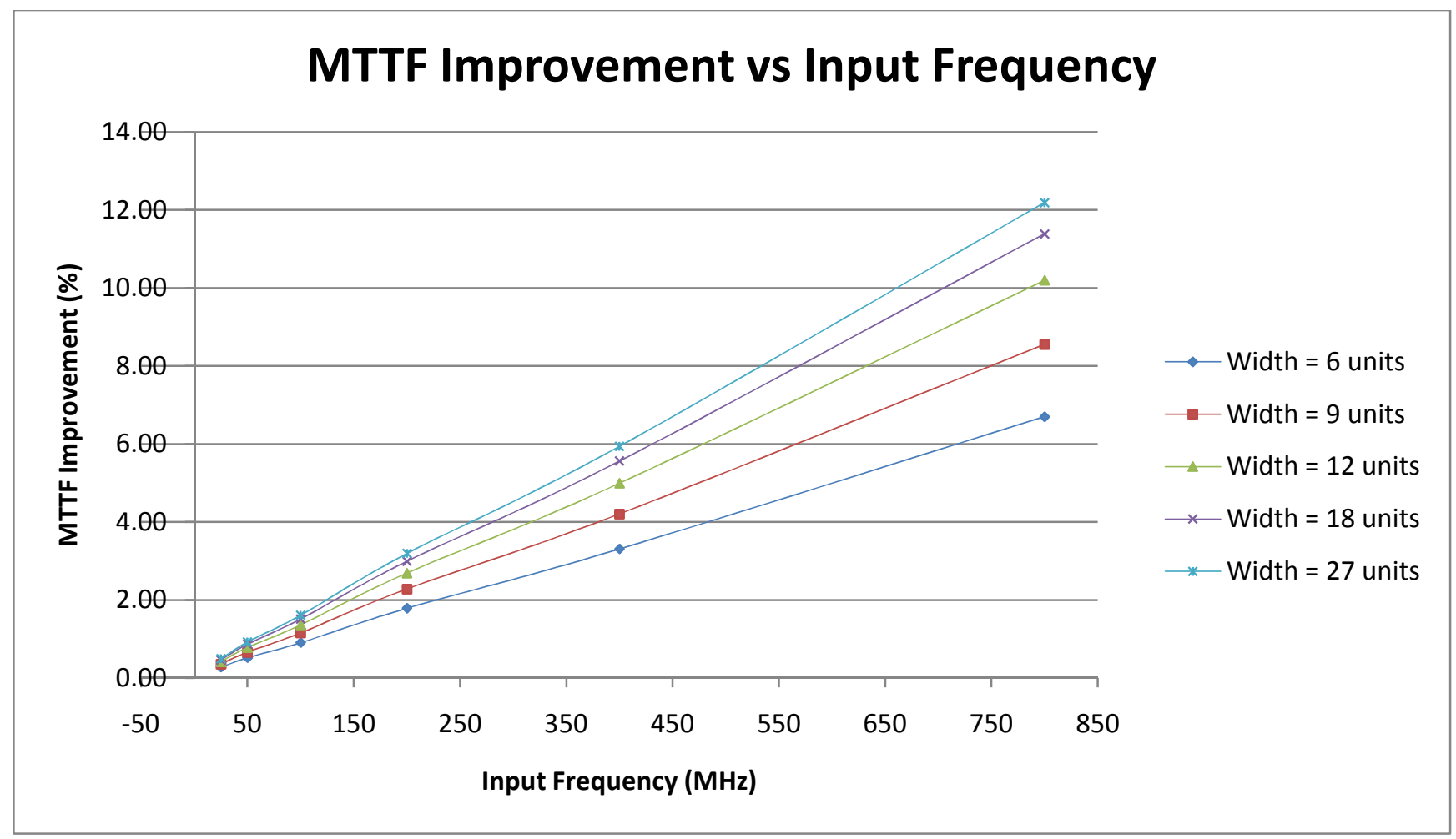

Figure 4.7: MTTF vs. Input Speed for Various Interconnect Sizing 


\subsection{Complex Circuits}

\subsubsection{Adders}

For this section, I tested two different adder types, Ripple Carry and Kogge-Stone. The adders were much more complicated and required a somewhat different test method. There are naturally such a large number of input/output combination pairs for each of the adders, thus testing them all would be cumbersome. Thus, inputs were carefully chosen in order to stress the various areas of the circuits equally. The ripple carry adder is one of the simplest adder types, and seeing as how all gate voltages were close to their nominal values, analyzing the data was straightforward. However, the Kogge-Stone adder is much more complex and there are several areas of the circuit that are relatively inactive compared to other. Current was sometimes highly concentrated in these spots and led to the failure of the device.

\subsubsection{Ripple-Carry Adders}

Three Ripple Carry Adders (RCAs) were designed for this section. They were 8, 16, and 32 bits in size. All three adders were constructed using the full adder block discussed in Appendix B. To effectively test the set of adders, the inputs were chosen so that the A and Carry-in signals were switching twice as fast as the B signals. This input method was able to equally stress all of the MOSFETs so that there was no inactivity throughout the circuit. The average current density found at the failure locations of the circuit match the behavior of circuits analyzed before; the faster the input speed, the higher the average current density, and the lower the MTTF.

As mentioned in prior sections, self heating is often a factor in the determining the failure time for the larger circuits. For the RCAs, they are all large enough allowing HotSpot to 
correctly calculate temperatures of a given block. The smallest subcircuit used for the RCA was the full adder. Using the input combination described above, all MOSFETs have close to the optimal average gate voltage and are stressed pretty much the same amount as one another. This makes it much harder to analyze, as one can't just say that a certain FET is on far more than others thus it fails first. Temperature then becomes a large factor in determining the adder's MTTF. The temperature of a particular region may not necessarily be the ambient temperature specified in the spice netlist. Naturally, the faster the circuit is run, the higher the temperature. Also, temperature increases can be seen with increasing adder size (in terms of total bits). Speed is also a huge issue primarily when it is too fast for the circuit to handle. At high input frequencies, the rise and fall times of a signal are often too quick and do not allow the voltage enough time to stabilize. As a result, pieces of the circuit remain inactive and cause the circuit to fail. There are also times where both NMOS and PMOS FETs will be try to turn on at the same time due to the instability of the gate voltages, which increases power consumption and ultimately temperature.

To make analyzing the RCAs more thorough and realistic, the frequency of the inputs was limited to $25 \mathrm{MHz}$, the highest frequency at which the output stabilizes before the next clock cycle. Any faster and inactivity skews the results. Figure 4.8 shows the MTTF against the different size RCA adders. For the 8 and 16 bit adders, the MTTF is virtually the same, as self heating is not yet evident. The very slight differences between the MTTFs of the two adder types are a result of increased current density in the 16 bit. However, the 32-Bit adder has a significantly lower MTTF because of the self heating affects. The is a very small temperature increase ( $<1$ degree Kelvin) in the 8 and 16 bit RCAs, but the temperature of the 32-Bit RCA 
raised over 5 degrees Kelvin. The self heating effects are even more apparent with increased rail voltage.

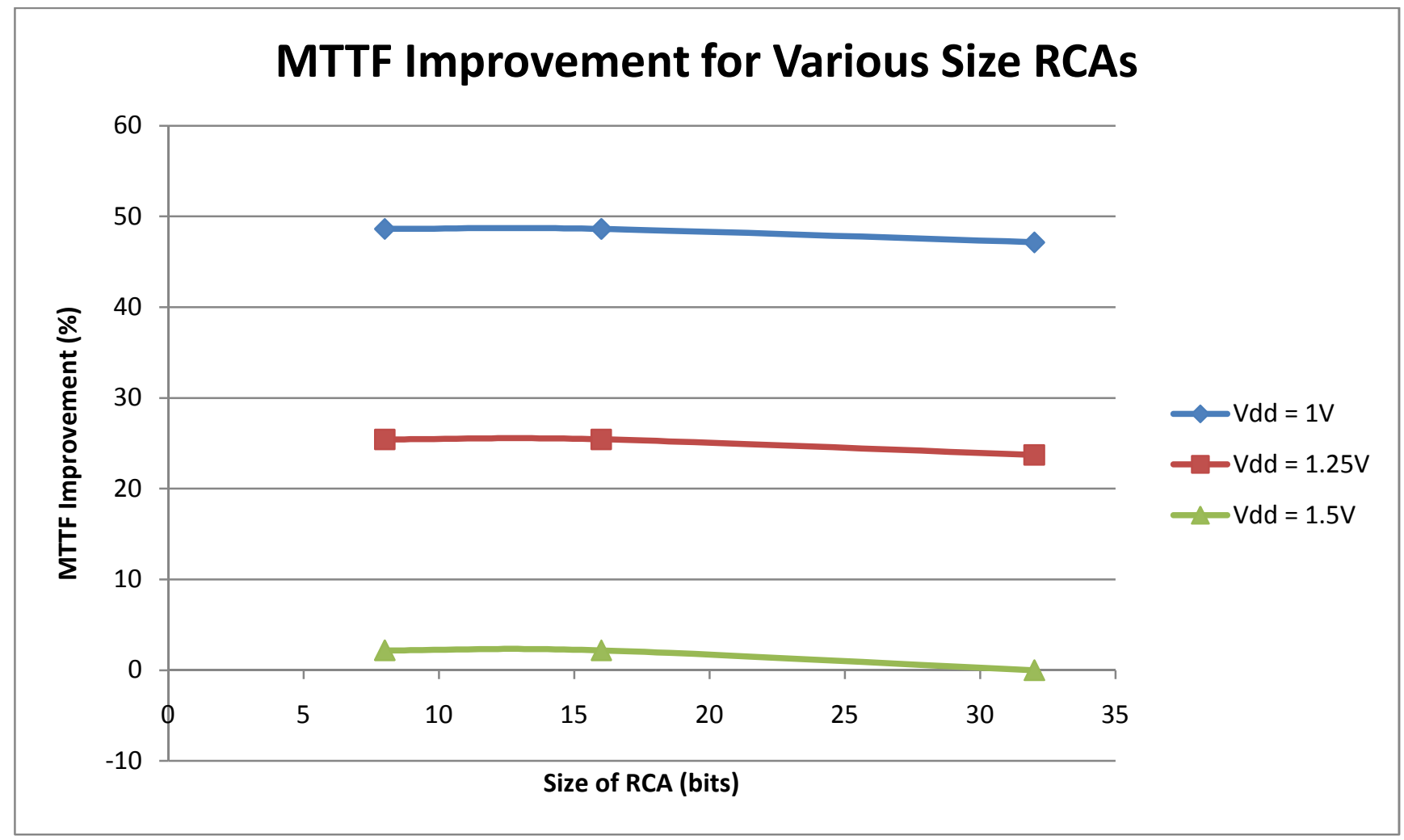

Figure 4.8: Comparison of 8-Bit, 16-Bit, and 32-Bit Adders

\subsubsection{Kogge-Stone Adders}

The Kogge-Stone adder is one of the more complex adders used today. Its operation and functionality is discussed in Chapter 3. I analyzed a 16-bit Kogge-Stone adder for this portion of the project using a similar test method as demonstrated with the RCAs. However, because of the added complexity in design, the input combination used for testing did not ensure that all FETs were stressed equally. Several areas of the circuit showed significantly more activity than others. This drastically reduced the MTTF of the entire circuit, making some failure times lower than their ideal value. 
Before getting into the analysis of the circuit, it is important to address a few points. First, for the lifetime of the circuit all of the elements would have 50\% activity (ideally) and that the same trends for other circuits would apply to this KS adder; one of the last stages will ultimately fail first due to the delay contribution of all previous stages. The critical path of the adder shown in Figure 4.9 tells me that the last XOR-gate will be the most likely to fail. Code was written specifically to focus on the last stage: each of XOR-gates for each of the sums.

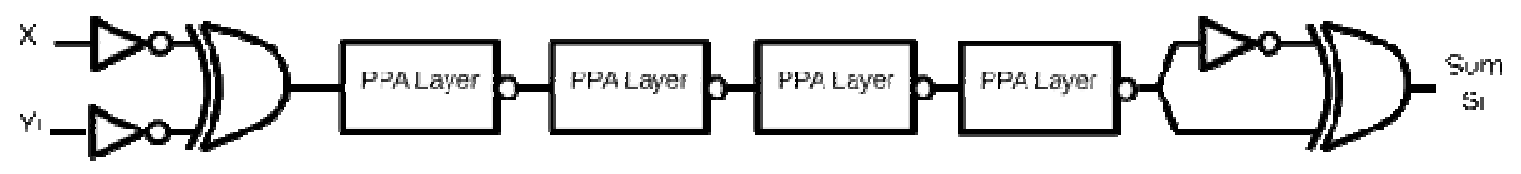

Figure 4.9: Critical Path of the KS Adder

Unlike any of the other circuits previously analyzed, the Kogge-Stone adder shows much higher current densities at the failure locations due to the constant activity of certain component, especially when run at higher frequencies. The increased current density causes an increase in temperature and makes self heating much more apparent. Power consumption is greater with the higher current density and temperature, thus the MTTF decreases tremendously. For my purposes, each subcircuit of the adder will be analyzed individually, and then the entire circuit will be looked at as a whole.

The Kogge-Stone consumed a great deal of power, which increased internal temperature up to 11 Kelvin depending on the rail voltage. If you look at Figure 4.10 you will see the affects of starting ambient temperature, rail voltage, and input speed. The y-axis is the MTTF improvement while the $\mathrm{x}$-axis is the average power consumed by the circuit. The different power values are obtained from simulating the circuit with different switching frequencies. As stated earlier, twenty-seven (27) different tests were run. In the graph, nine (9) lines are plotted and 
each line contains three points. Each line represents the lifetime improvement of the circuit at three different input frequencies. Temperature and voltage for each line is held constant as can be seen from the graph below. As the rail voltage is increased the MTTF further decreases with increased current density. The higher the rail voltage, the more power the circuit is able to consume which is shown by the last point in each line being plotted further to the right. As with all the other circuits, the higher the input frequency, the lower the MTTF, as input frequency increases temperature and associated current density. In Chapter 5, current density and temperature will be analyzed separately using this data.

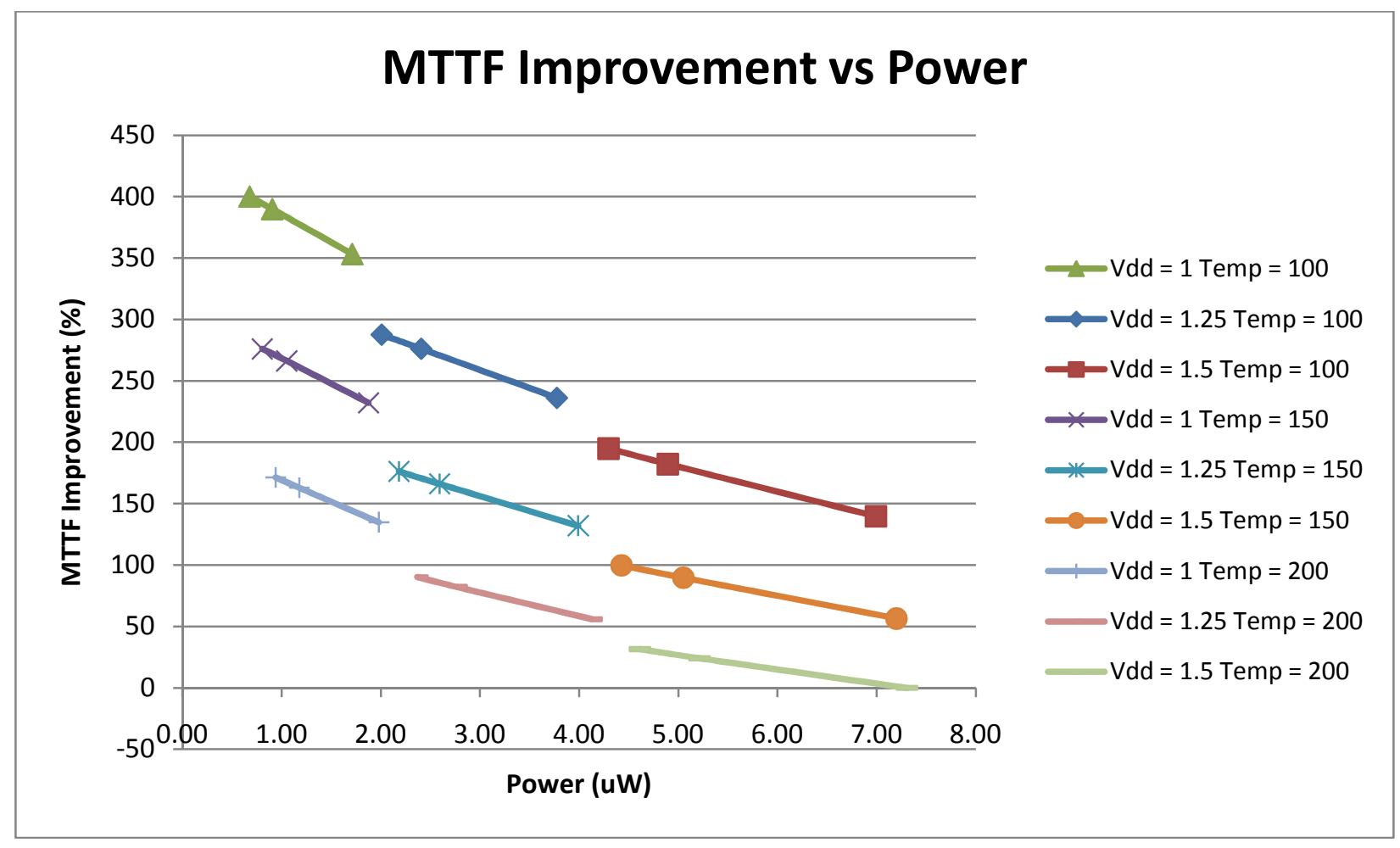

Figure 4.10: Effects of Starting Ambient Temperature, Rail Voltage and Input speed for Kogge Stone Adder

\subsubsection{8x8 Register File}

The register file was the largest of the circuits implemented for this project. Although the design is fairly simple, the analysis is not so easy. Rather than focus on an input scheme that 
equally stressed each piece of the circuit (becomes a lot harder to do as the circuits get bigger), I decided to go with a more basic input scheme that is geared more towards the functionality of the device. Because it was impossible to test all input combinations, some of the data is slightly skewed, however this does not deem it as useless. In fact, the data collected for this register file is very useful, as it allows me to verify my results found for the smaller circuits, as they are the building blocks for any current semiconductor device. The circuit elements that have very low MTTF due to inactivity will be ignored for the analysis and my focus will be on the areas of the circuit that better allow me to verify previously seen trends, as well as discover new ones.

Due to the excessively large size of the register file, there was temperature variation throughout the device. Some areas will be much hotter than others, and in turn most likely to fail first. Data shows that the hottest areas of the register file were the SRAM cells, the inverters around these cells, the decoders, and the MUXs respectively. The individual bit cells cannot really be redesigned as they hold static data, however, the design of the 3:8 decoders and 8:1 MUXs can be re-evaluated to improve the circuit's overall failure time.

Although the bit cells are essentially "holding" the data, it is the MUXs and the decoders that are really doing the most work. They not only have to specify correct address lines, but are responsible for all output data. Current density in these regions is very high, and this along with the increased internal temperatures, causes these regions to fail. Also, the majority of the metal lines are distributed throughout these areas. My analysis will focus on the 8:1 MUXs and the 3:8 decoders found throughout the register file. The weakest MUX is found at the end of the circuit, near the output. The MUX used for the register file was created by feeding two 4 to 1 MUXs into a 2 to 1 MUX. For the analysis, the output pMOSFETs for the MUX subcircuits will be looked at. The decoders used for the register file were designed using a combination of AND gates and 
inverters. The failure locations for the decoders are the inverters at the output of the AND gates; given the ideal input, the output of an AND gate is low $75 \%$ of the time. One can conclude that the PMOS of the output inverter is the weak link.

The register file is a fairly simple design and is entirely made up of various subcircuits. The complexity only comes into play when the many subcircuits are tied together and operate as a function of one another. By analyzing the subcircuits that make up the register file, the reasons for the circuit's failure become clearer. Again, this is the largest of the circuits analyzed for this project. All factors affecting MTTF must be considered. There is a temperature gradient across the entire circuit, where self heating is more apparent than in any other circuit I have analyzed. Although a portion of the circuit may tend to operate at a rather cool temperature, there is often an element close by that does not. The high temperature of the associated area contributes to the heat found in other locations. It is the hotter areas in the circuit that I care about, as they consume large amounts of power and ultimately lead to a low MTTF. On the contrary, the cooler circuit elements help keep the overall temperature of the device down by distributing the heat, increasing the MTTF.

\subsection{Failures, Associated Performance Flaws and Potential Fixes}

Electromigration is an issue for two main reasons: high temperature and high current density, or in my case, high average current density. Sometimes, the effects on MTTF due to one factor may outweigh the effects seen by the other. Yet, almost all of the time, these two factors go hand in hand, as a higher current density usually means a higher temperature. The increased temperature means more power consumption, bringing down the MTTF for the entire circuit. 


\subsubsection{Input Frequency}

Input frequency is defined as the switching frequency of the fastest input for a given circuit. This greatly affects the MTTF of a device. Often times, the input is too fast and does not give the voltage enough time to stabilize and reach its full potential. The average gate or "turnon" voltage for a FET is always half the rail voltage. If the input frequency fails to allow the voltages to reach their ideal values, the circuit will not behave properly. As the circuit goes through its critical path, the later stages may not even function and the device is useless. Or the device may somewhat function but will fail quicker than it should.

\subsubsection{Skin Effect}

The skin effect is the tendency of an alternating electric current (AC) to distribute itself within a conductor so that the current density near the surface of the conductor is greater than that at its core. That is, the electric current tends to flow at the "skin" of the conductor. The skin effect causes the effective resistance of the conductor to increase with the frequency of the current [28]. At higher frequencies, current density greatly increases which causes the MTTF of a device to decrease. As mentioned in previous sections, high current densities have many undesirable consequences. Most metal lines have a positive, finite resistance, causing them to dissipate power in the form of heat. I already know that temperature greatly effects the lifetime of a circuit. At higher temperatures, the metal lines can melt or burn-up. Thus, keeping the current density sufficiently low is very important as it minimizes the amount the material forming the interconnect moves, which is electromigration. In today's technology, semiconductor devices are constantly being reduced in size, and although they demand less current, there is trend toward higher current densities to achieve higher device numbers in ever smaller chip areas. Moore's Law has continued to show this for several years. 


\subsubsection{Ideal Current Density}

In a perfect world, semiconductor devices would be designed to achieve zero current density. All elements of a device would function just as they should, turning on only when they need to, and turning off when not in use. Current would be held to an absolute minimum throughout the entire device. With this, the metal lines associated with each FET would also be optimized. Area would not be a concern and cross sectional area for interconnect material would have no sizing limits. However, this is impossible for any designer to achieve and thus it is important to simply focus on minimizing current density. This also becomes harder to do as the complexity of the circuit increases. To see just how much this affects failure time, I can look at the Kogge Stone adder. Figure 4.11 shows the MTTF improvement versus the input frequency of the circuit if the average current density of the interconnect material were at its optimal value of zero as compared to simulated data. The data assumed a starting ambient temperature of $373 \mathrm{~K}$. All three rail voltages were plotted to better show these trends. For the simulated case, high current densities tremendously affect circuit performance and bring down the MTTF by several years. With the high current density comes an increase in temperature, and the affects of self heating become more obvious. However, in the ideal case where current density is zero, the only limiting factor on MTTF is temperature. Temperature is naturally lower with smaller current densities, and self heating is less apparent. With the ideal case, MTTF is almost the same for all 3 rail voltages. However, using the simulated data, the rail voltages naturally increase current and in turn current density, so MTTF changes significantly. 


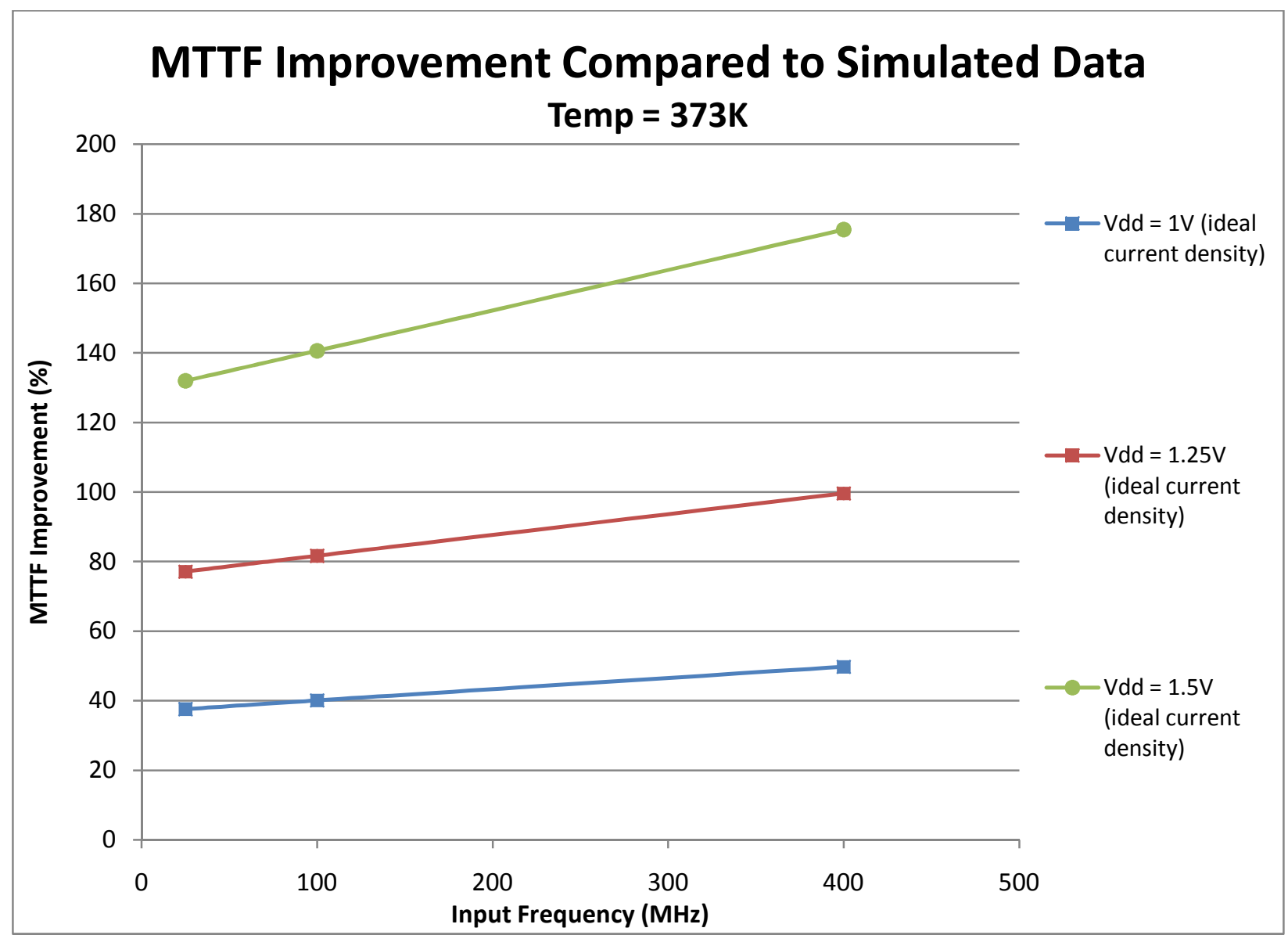

Figure 4.11: Input Frequnecy vs. MTTF when Temperature $=373 \mathrm{~K}$

As ambient temperature is increased, self heating becomes more of an issue. However, the effects of self heating are still not enough to outweigh the effects of high current densities. Figure 4.12 below is a similar graph to the one above, but assumes a starting ambient temperature of $423 \mathrm{~K}$. 


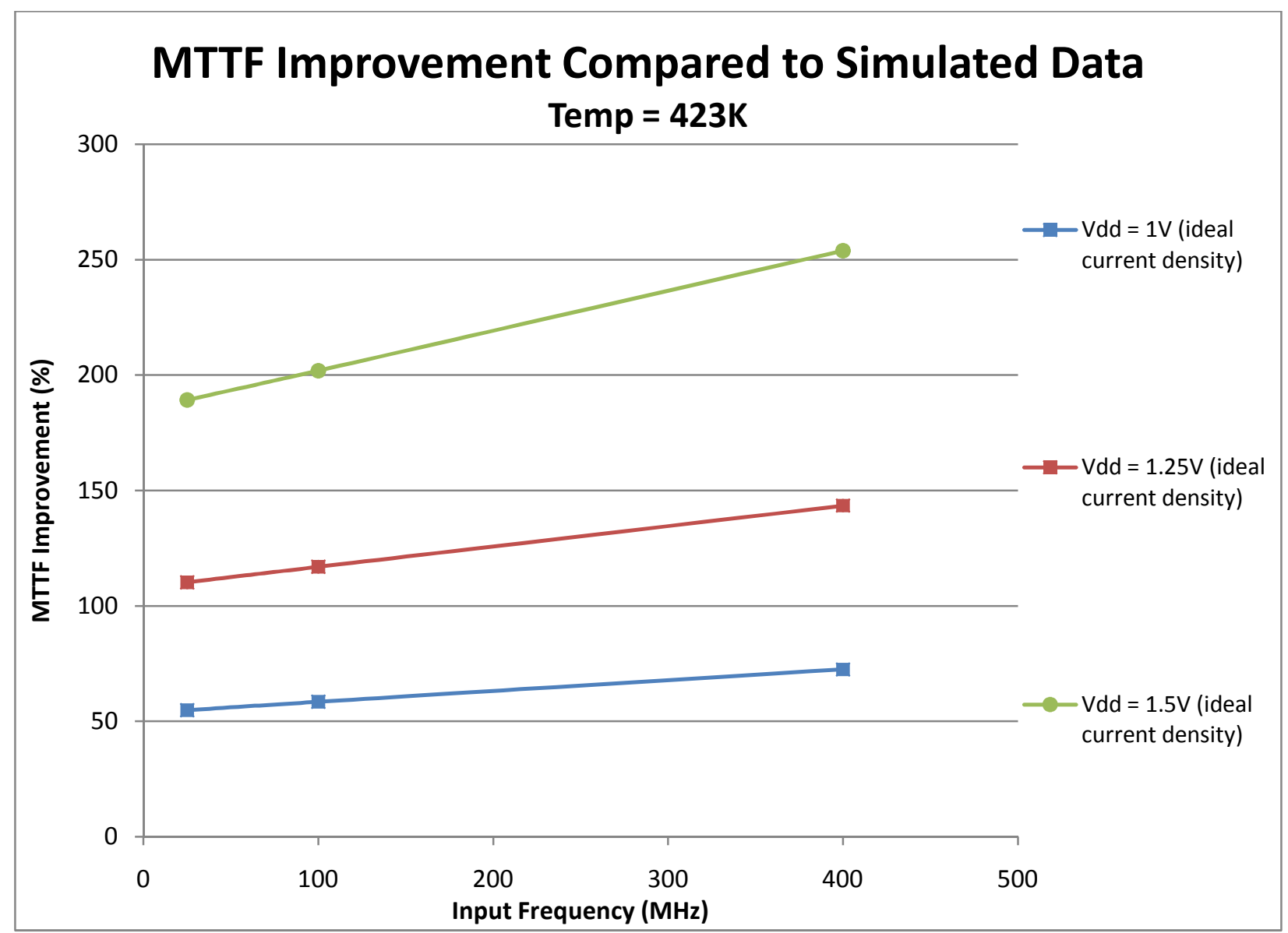

Figure 4.12: Input Frequency vs. MTTF when Temperature $=423 \mathrm{~K}$

This graph looks remarkably similar to the corresponding one for $373 \mathrm{~K}$, however there are some notable differences. As temperature is increased, self heating has a greater impact on the circuit's lifetime. This, combined with the much higher current densities, drastically reduces the MTTF of the device. Looking carefully at the two graphs, one can see that the effects of increased rail voltages are less severe at the higher temperatures. Current density and temperature take over and control the circuit's lifetime. For the ideal case, the changes in MTTF are minimal, as current density is zero. I still have self heating to worry about, yet its affect is at most $14 \mathrm{~K}$ on the internal device temperature, not enough to really decrease MTTF by more than a few years. 


\subsubsection{Interconnect Sizing}

The width of a given interconnect directly affects the current density through it. Increased interconnect width allows for a reduction of current density through the metal lines. Although current through the MOSFET is the same, the wider metal width reduces its density. With the reduction in current density comes a slight decrease in power consumption yet a higher MTTF. At high current densities $\left(>10^{6} \mathrm{~A} / \mathrm{cm}^{2}\right)$, essentially doubling the interconnect width halves the current density. There is now a wider area for electrons to flow, and this helps prevent the formation of voids and hillocks that eventually crowd the line and cause it to break. It is important to note that increasing or decreasing the interconnect width does not change the current, ONLY the density of that current. Below is a graph showing the MTTF of an inverter driving a load, with different interconnect sizing at source and drain metal lines. Simulations were run with an input frequency of $25 \mathrm{MHz}$ and rail voltage of $1 \mathrm{~V}$ and at $373 \mathrm{~K}$.

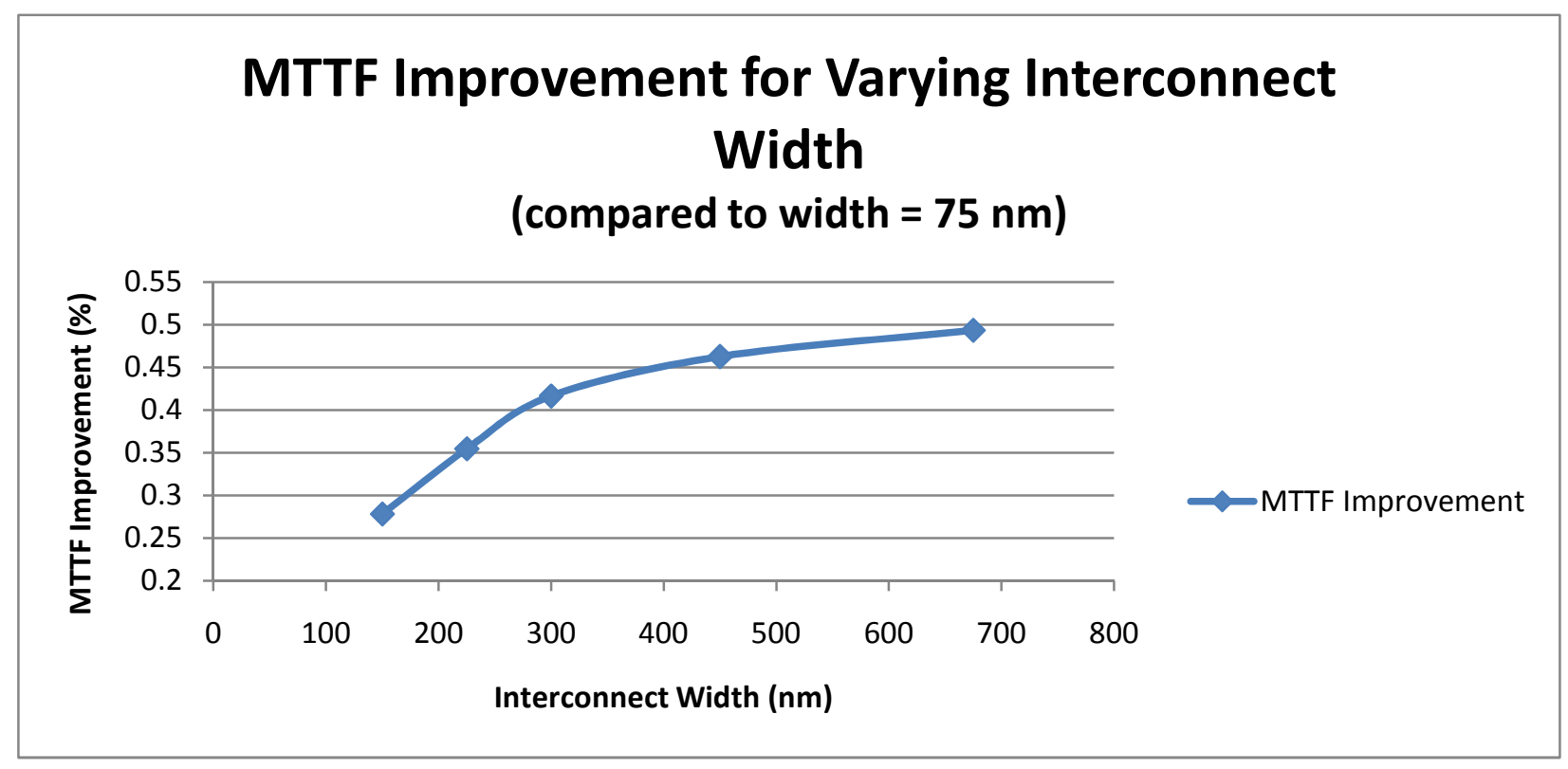

Figure 4.13: Representation of Varying Interconnect Sizing 
As you can see, changing interconnect sizing for such a small circuit doesn't really affect the MTTF. Yes, the MTTF does increase a bit but it's a negligible amount. Also, for the model created to calculate MTTF, there is a constant in front of the equation whose value is 308.64 ; this being multiplied by the exponential relationships current density and temperature has on MTTF. Again, electromigration will not occur unless current density is ultimately high enough to break the metal line. In larger circuits, current density can approach values $>10^{\wedge} 7$ and electromigration effects greatly damage a device. How you design the smaller circuits that make up the more complicated designs is crucial in prolonging the lifetime of the device.

\subsubsection{Temperature and Resistivity}

Temperature greatly affects the lifetime of a circuit. Increased temperature can lead to higher current densities and increased resistivity of the metal lines. With today's scaling down process, the thickness of metal lines is often reduced, which also adds to the increased resistivity of the interconnect material. The higher temperature and associated increase in resistivity exacerbates problems with power consumption and signal delay. Circuit's run much slower than they should. Clearly, decreasing the resistance and resistivity of circuit materials may reduce power consumption and increase the speed at which a circuit switches. Below are figures 4.14 and 4.15 showing the RC time delay between the input and output of an 8 bit RCA. The first has a starting ambient temperature of $373 \mathrm{~K}$ while the second runs at $473 \mathrm{~K}$. 


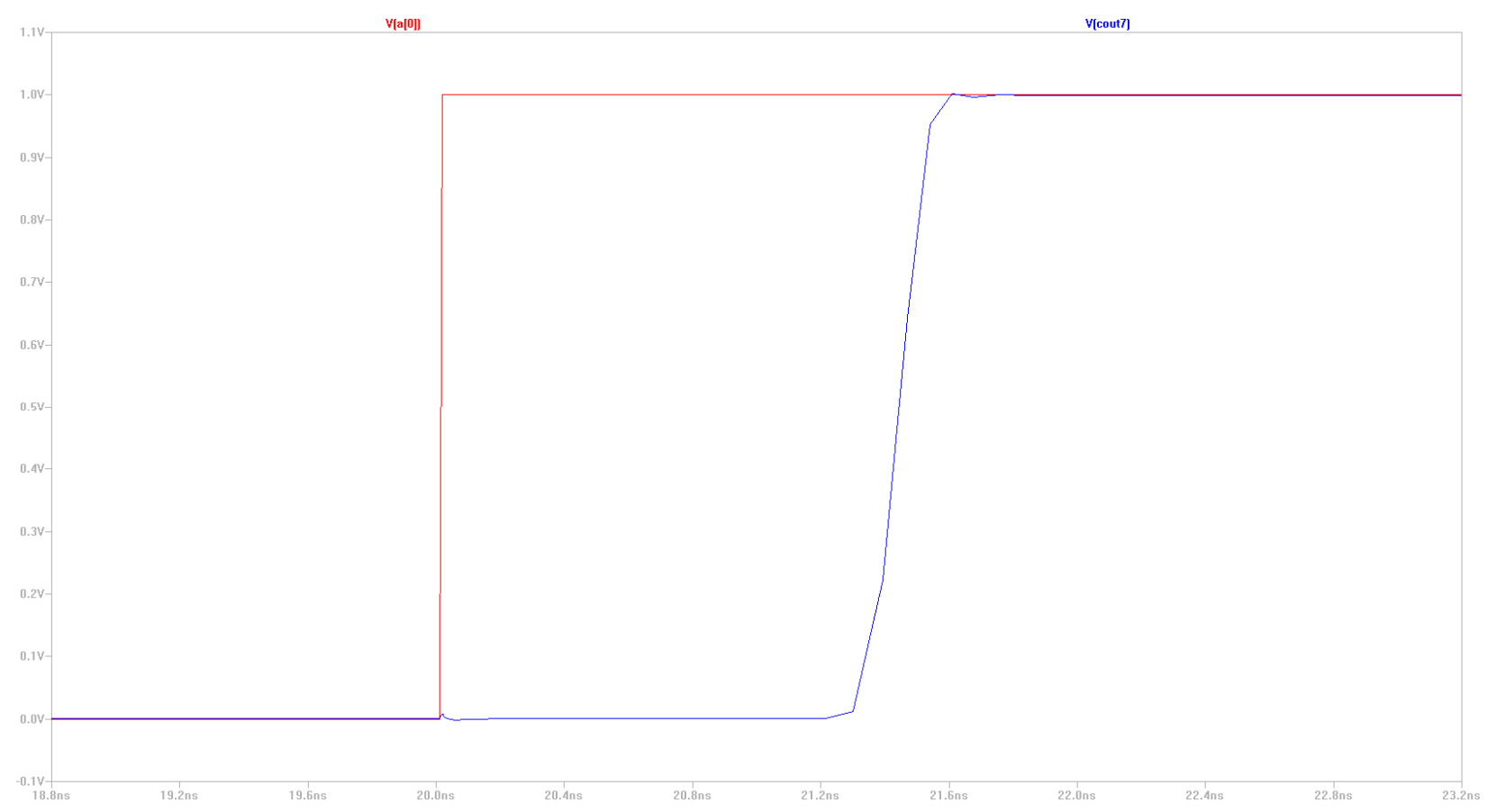

Figure 4.14: $\mathrm{RC}$ time delay for 8 Bit RCA ( Delay is $1.43 \mathrm{~ns}$ temp $=200$ )

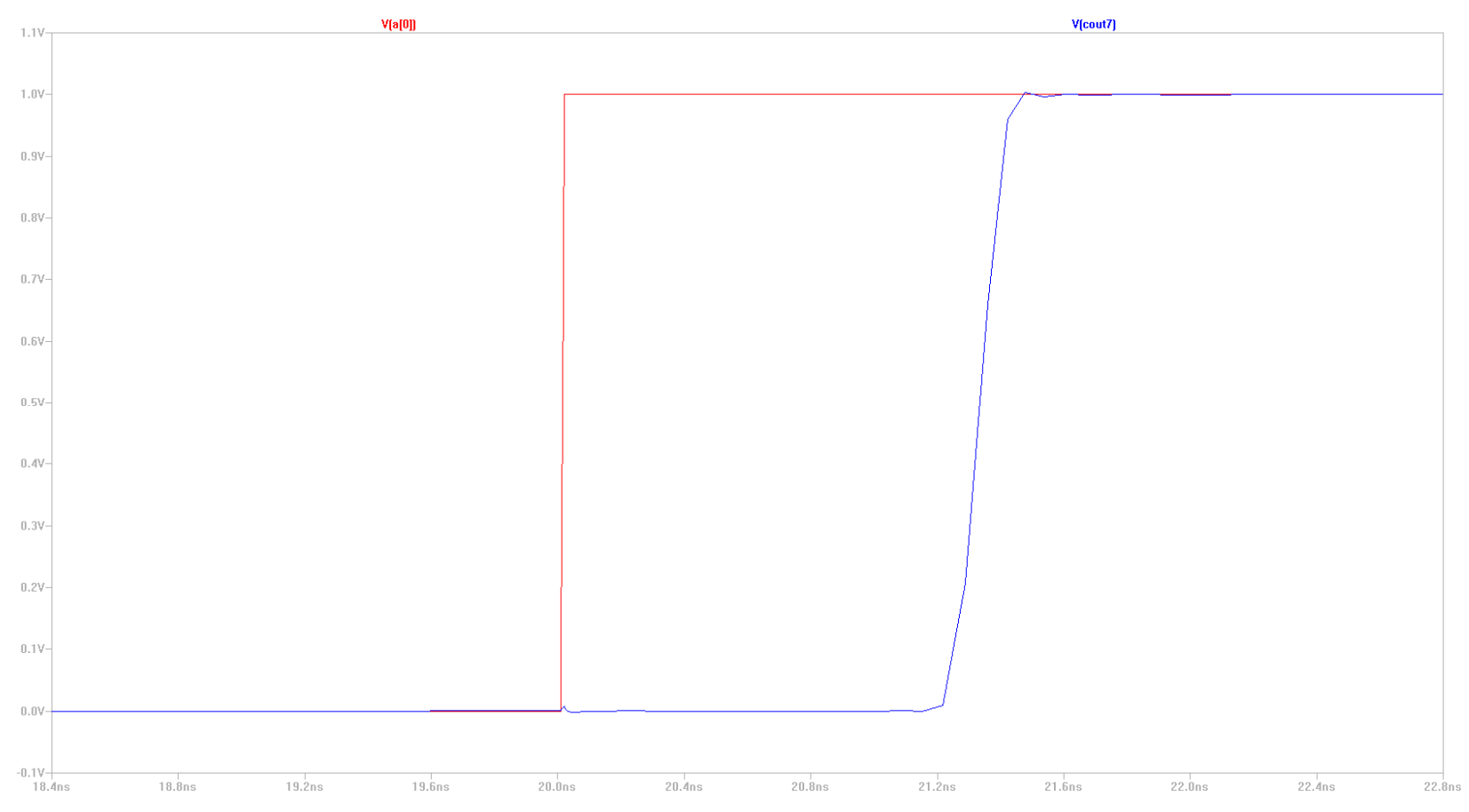

Figure 4.15: RC time delay for 8 Bit RCA (Delay is $1.32 \mathrm{~ns}$ Temp $=100$ ) 
The changes in delay are a bit hard to see, but the numbers show that with the increased temperature there is an added 110ps of delay. The increased resistivity at the higher temperature slows down the circuit and consumes more power, causing a decrease in MTTF.

Switching frequency is also a large factor impacting temperature, as the faster a circuit is ran, the hotter is gets. More power is required and overall failure time decreases. Clearly, decreasing the clock frequency of a circuit reduces power consumption and temperature, prolonging the lifetime of the device. I also have to consider the idea of self heating. A temperature increase as a result of self heating can be detrimental to a circuit's lifetime. Self heating tends to be more apparent at much higher temperatures and can often offset a section's ambient temperature by up to 25 degrees [26].

As done in earlier sections, I can look more directly at temperature's impact on MTTF. Figure 4.16 below shows MTTF vs Input speed for the weak link XOR gate of the Kogge-Stone adder at $373 \mathrm{~K}$. The graph illustrates simulated temperature and also constant temperature (no self heating). As can be seen, the effects of temperature on MTTF are noticeable, but the change in MTTF due to temperature is not nearly as significant as that with current density. It can also be seen that at higher rail voltages the changes to MTTF are more severe, as the circuit consumes more power. 


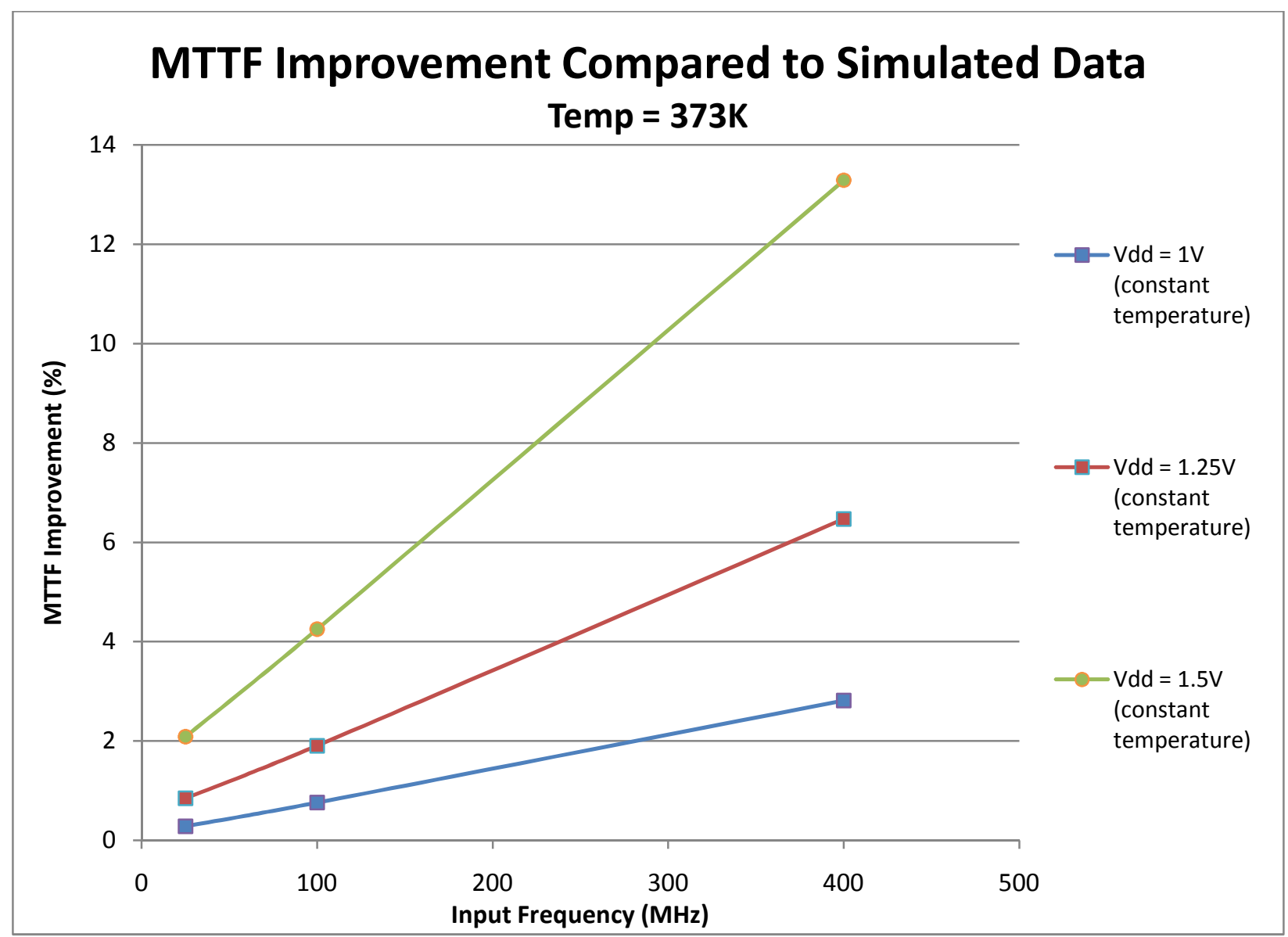

Figure 4.16: MTTF vs. Input speed at Simulated and Constant Temperature (weak link XOR of KoggeStone)

Increased temperature does have its drawbacks; however, on the smaller circuits that act as building blocks for the more complicated designs, the effects are not that severe. Also, the changes in current density as a result of increased temperature are not large enough to really drop the MTTF. Figure 4.17 shows the MTTF for the weak link XOR gate for the Kogge-Stone adder when compared to simulated data. The graph assumes constant current density (same value for all 373, 423, and 473 Kelvin) with increased temperature. There are no huge differences in the MTTF of this XOR gate for each of the starting ambient temperatures. As a result, the improvements made to designs in Chapter 5 will not focus on running the circuits at higher 
starting ambient temperatures, but more on the results on increasing temperature as a result of self heating.

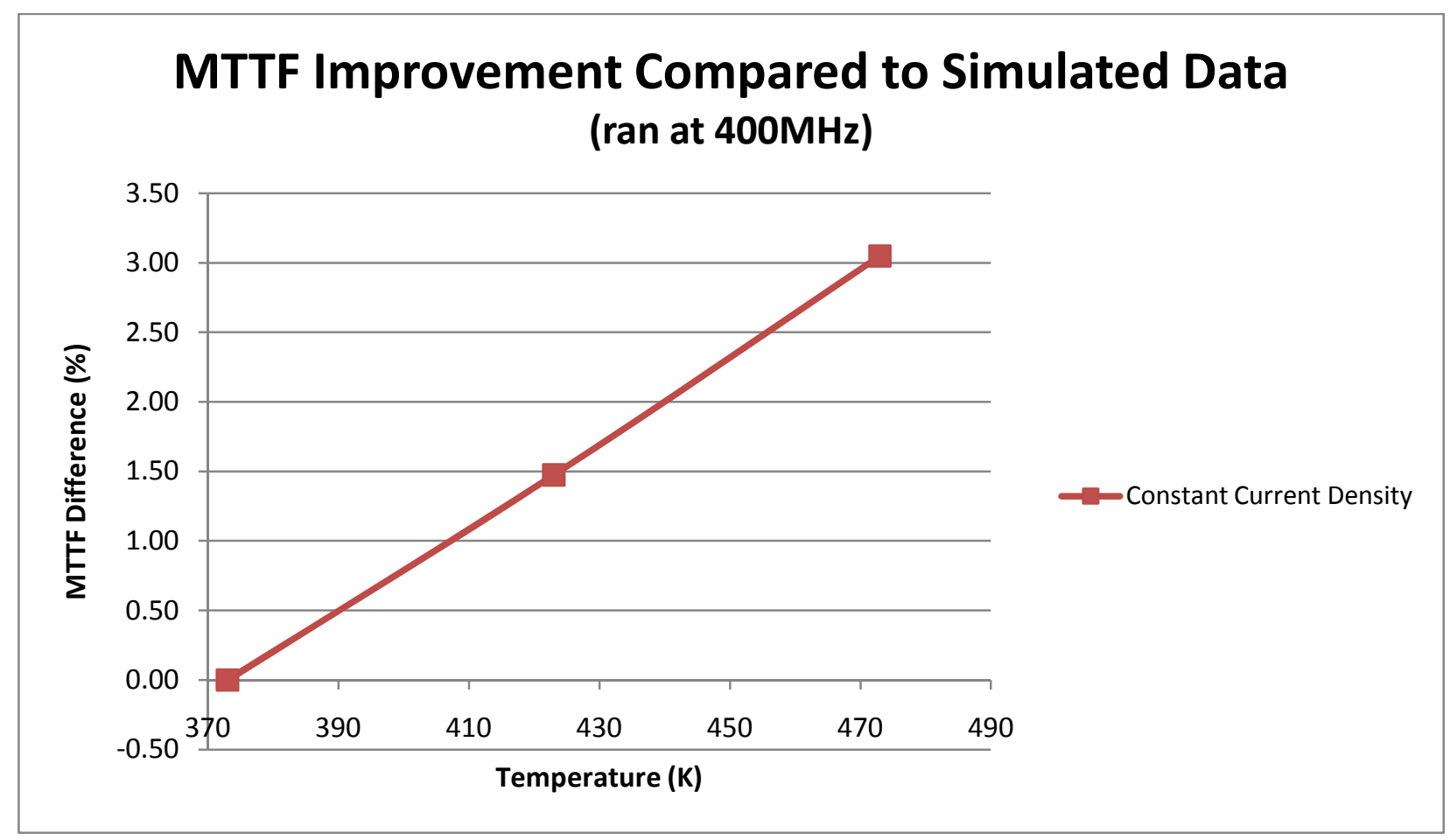

Figure 4.17: Graph Showing MTTF for Constant Current Density at Varying Starting Ambient Temperatures 


\section{Chapter 5. Improving Design and Ways to Minimize Failure}

The previous chapters show me a very detailed overview of electromigration and also examine the detrimental effects it can have on a circuit's lifetime. It is known that the failures associated with electromigration occur inside the interconnect material and are a result of electron crowding in various areas within the metal lines. This chapter examines ways to improve these failures and prolong the lifetime of any given circuit.

Temperature and current density are the two biggest factors to analyze when dealing with electromigration. Although temperature can be somewhat regulated, for the most part its actions are out of the designer's control. Current density can however be greatly reduced with careful design considerations. Increasing metal line widths at locations where current density is highest can yield a large reduction in current density (at high current densities, doubling interconnect width halves current density). For my first improvement, I will look at increasing the metal of the power rails. It is often the metal lines here that experience the highest current densities, as current flows from power to GND, and is strongest at the source. This method may or may not work, depending on the circuit's behavior. Current distributes itself across a device based on the operation of that device, and simply increasing the width of the power rails may not be enough.

Thus for my next improvement, I will look at increasing the metal lines at the outputs of a given circuit. With any design, there are always stages driving other stages. Current must have a clear path from the output of one device to the input of the next in order operate efficiently and effectively.

The above changes primarily change the current density component of the MTTF model. With any alteration to a device comes tradeoffs, be it power consumption, temperature increase, 
higher delay etc. Low power is of utmost importance in today's technology, and methods like lowering the rail voltage and decreasing the clock frequency can help minimize power, but both of those changes will drastically affect performance.

However, some changes must be made to improve the overall lifetime of the circuit. Again, with the continued scaling down process of semiconductor devices, all improvement methods must be carefully examined. They become harder and harder to analyze, but as with any device, a careful design process is always beneficial to the lifetime of the circuit.

\subsection{Improving MTTF of the XOR Gate}

The XOR gate is the simplest "complete" circuit in the catalog of circuits tested. A complete circuit in context of this project will be any circuit having at least two stages, with the first stage affecting the second, etc. The improvement methods above will first be tested on the XOR gate to see in fact if MTTF is increased, and also examine the tradeoffs are a result of these improvements. Once again the weak link was N1.

\subsubsection{Increasing Interconnect Width}

One improvement technique was to double the interconnect sizing at the source/drain locations of the weak link. Doing so halves the current density and should thus improve device failure time. With the XOR gate, current densities are still fairly small due to the simplicity of the gate type. Current density did decrease by $50 \%$ and there was a slight increase in MTTF. Current densities are in the low thousands and electromigration is not really an issue, hence the only slight increase in failure time. There is a more noticeable increase in MTTF at faster input frequencies due to the accompanying higher current densities. Also, the gate was only run at $373 \mathrm{~K}$ and self heating is not a huge issue. Parasitic capacitance is also slightly reduced; however 
the small changes have little effect on MTTF; larger circuits may show stronger improvements with the lower capacitance. There were no performance drawbacks with the increased metal sizing. Again, I was only targeting the weak links of the smaller circuits. In larger designs, where more substantial changes are made, performance tradeoffs may become an issue.

Another technique involved increasing the metal width of power and ground lines. This technique had the same effectiveness as increasing the interconnect width at the source/drain of the weak link, but only proved effective if power and ground rails were in fact the sources/drains of the weak links. If not, there was no change to MTTF or to the overall performance of the circuit.

\subsubsection{Decreasing Size of Weak Link}

Another improvement technique was to decrease the gate size of the weak link by $1 / 2$. Doing so not only reduces capacitance at the gate but also lowers the rise/fall times. Decreasing the width of the gate weakens the FET and slightly lowers current density (more so at faster input speeds). MTTF changes very slightly, but it is an increase. There are some noticeable performance tradeoffs as well. The smaller gate size slows down the circuit and actually consumes slightly more power. Typically slowing down the circuit would reduce power consumption, but the single change may not be all that beneficial.

\subsubsection{Improving Circuit Speed}

The last improvement technique was to increase the speed of the circuit by increasing the $\mathrm{P}$ and N MOSFETs of the stage driving the weak link. The FETs were increased to 2x in size and there are some very noticeable improvements. The XOR gate is not affected by this change as the weak link was the first stage in the circuit. In general, this increases the current going through 
the driving gates but decreases the amount of current through the weak link. This allows for a lower current density and improved MTTF. This modification however comes at the cost of power consumption. Although the delay of the circuit decreases, there is a significant increase in power usage. All of the mentioned changes however focus on designing the circuit around the weak link. The weak link characteristics are improved, but a new weak link is then found. In larger designs, changes will focus more on the performance of the entire circuit, now that weak links and associated improvements can be made.

\subsubsection{Failure Time Improvements}

Figure 5.1 shows the MTTF improvement of the XOR for various changes. Changing the source/drain metal line sizes at the weak link proved to be the most effective, as this change provides the largest reduction in current density. Decreasing the size of the weak link was also beneficial to the circuit's lifetime. You can also see that changing the size of power and ground metal lines had no effect on the MTTF for the gate. As mentioned earlier, this particular change is only beneficial if either the source/drain of the weak link is in fact VDD/GND, in which case the change is essentially the same as that mentioned above. The improvements are seen more at the slower circuit speeds as well. The bar graph clearly shows the improvements on failure time with the increase of S/D metals and reduction in gate size of the weak link. More importantly, one can see that the increased sizing of power/ground lines had no further improvement on MTTF than what was seen with the changes to S/D locations. 


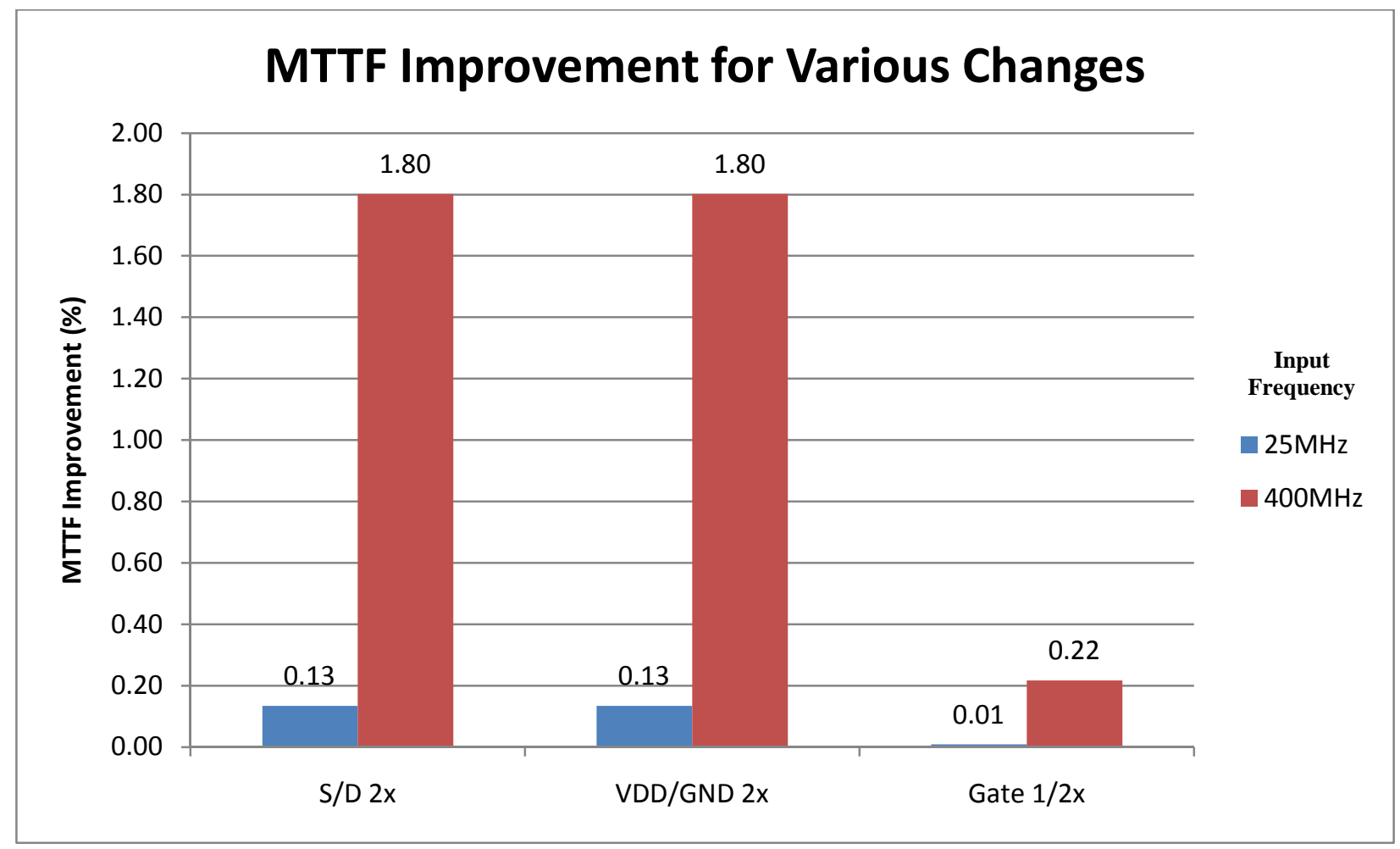

Figure 5.1: MTTF Improvement of XOR Gate for Various Changes

\subsubsection{Affects On Power and Delay}

Figures C.1-C.4 in Appendix C show the effects on delay and power consumption for various changes to the XOR gate, MUX, and Full Adder, again for the two different input frequencies of $25 \mathrm{MHz}$ and $400 \mathrm{MHz}$. They will be referred to several times in the next few sections. For the XOR, delay stays the same for the majority of the changes, increasing slightly with the decreased gate size of the weak link. Power consumption also remains the same for the majority of the changes, again increasing slightly with the reduced gate size. Ideally, power should decrease a bit as the circuit is running slower. However, the input to the failing FET is one of the two inputs to the XOR gate; it is a perfect square wave at $50 \%$ duty cycle and has no internal logic to change its operation. With that said the current running through the FET doesn't change that much, decreasing only slightly. This yields a slightly smaller current density, but essentially applies more stress to the FET, causing it to use more power. All changes do increase the MTTF. 


\subsection{Results on Larger Circuits}

The following sections examine modifications to much larger circuits, such as the Kogge Stone adder and Register File. For the larger circuits, changes will be made to improve the overall lifetime of the circuits, and not just the weak links.

\subsubsection{Kogge-Stone Adder}

The changes made to the Kogge-Stone Adder were geared towards improving the lifetime of the entire device. Thus the changes weren't nearly as specific as the smaller circuits. The majority of weak links for the Kogge-Stone Adder were the AND and XOR gates. These gates are responsible for driving the majority of prefix logic the adder requires. Thus, for FIX1, the AND and XOR gates were doubled in size, and the prefix logic was reduced to half its original size. Also, all source/drain metal lines were doubled in width. This only yielded a small increase on MTTF and caused the circuit to consume more power along with an increase in delay. FIX2 doubled metal size, kept the PPA blocks their original size and decreased the size of the XOR and AND gates by about 30\%. This fix showed a significant increase in MTTF without changing the delay much, but did consume more power. FIX3 again doubled metal size, increased the size of the PPA blocks, XOR and AND gates. This led to a similar rise in MTTF as seen for FIX1, used more power but made the adder faster. The last and final fix was very specific to the weak link of the adder. It involved increased metal size, and only decreasing the size of the weakest XOR gate. This show the largest improvement to MTTF, used much less power and kept delay the same as it was before the fixes. Figure 5.2 below shows the individual subcircuits with and without FIX1, just to give you an idea of what I were trying to achieve. 


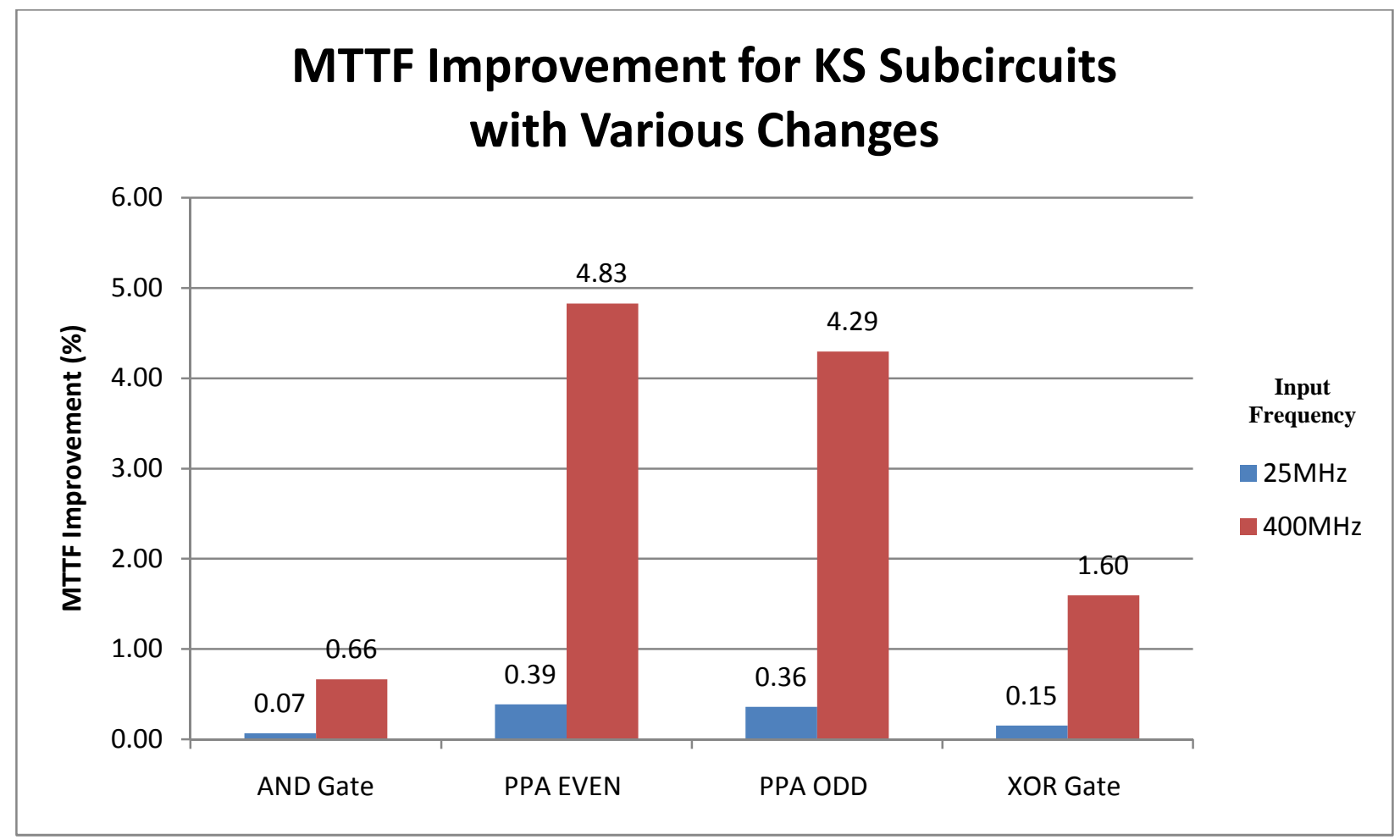

Figure 5.2: Improvements for Subcircuits of Kogge-Stone Adder

The above graphs only show the effects on FIX1 for the subcircuits, just to give you an idea of how I seek to increase MTTF. There are a variety of ways to do this; it's just a matter of discovering which method is the most effective. Figures 5.3 and 5.4 show the effects on MTTF, power, and delay for the entire adder using the fixes mentioned above. 


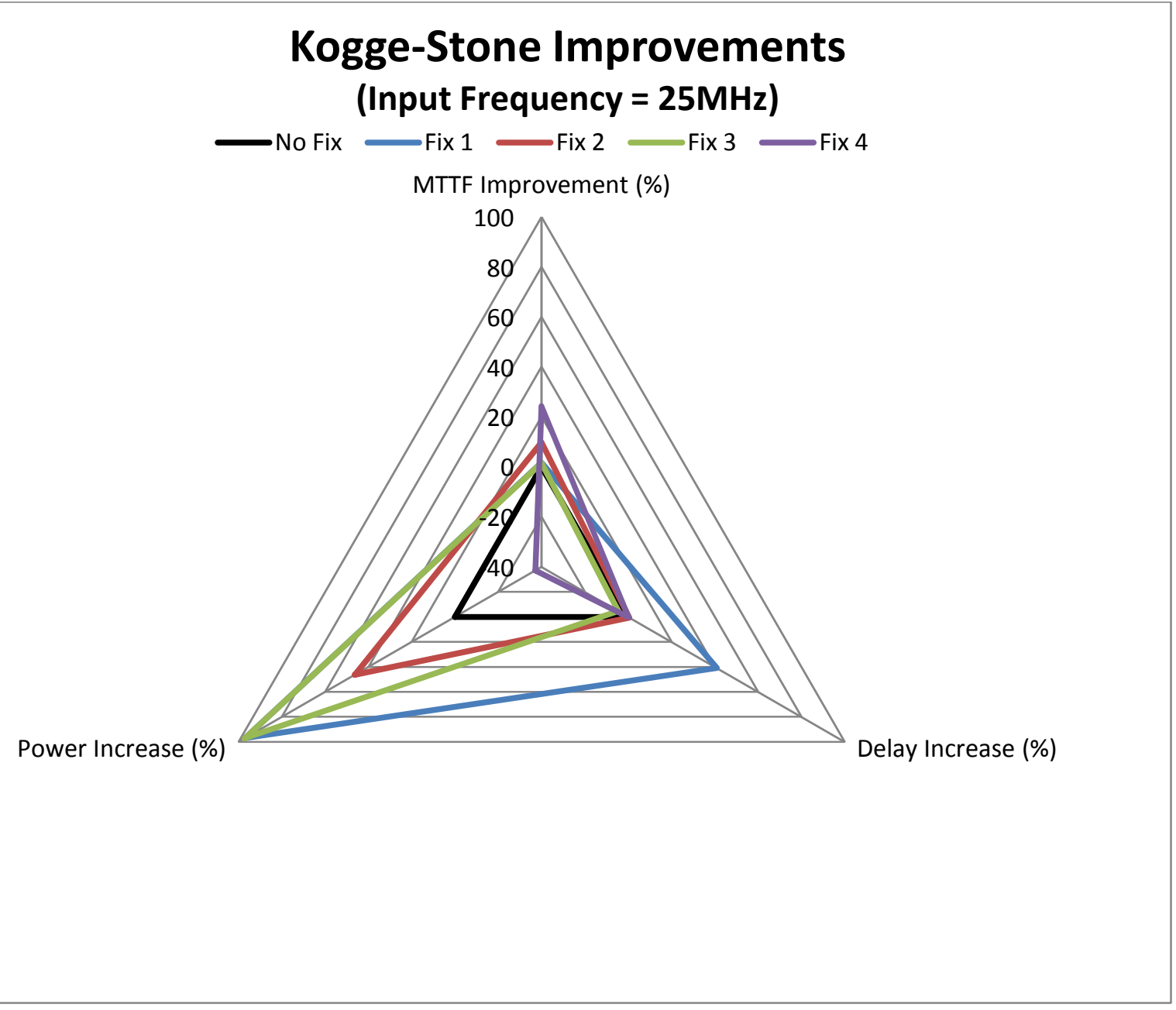

Figure 5.3: Results on Kogge-Stone Adder for Various Fixes @ 25MHz 


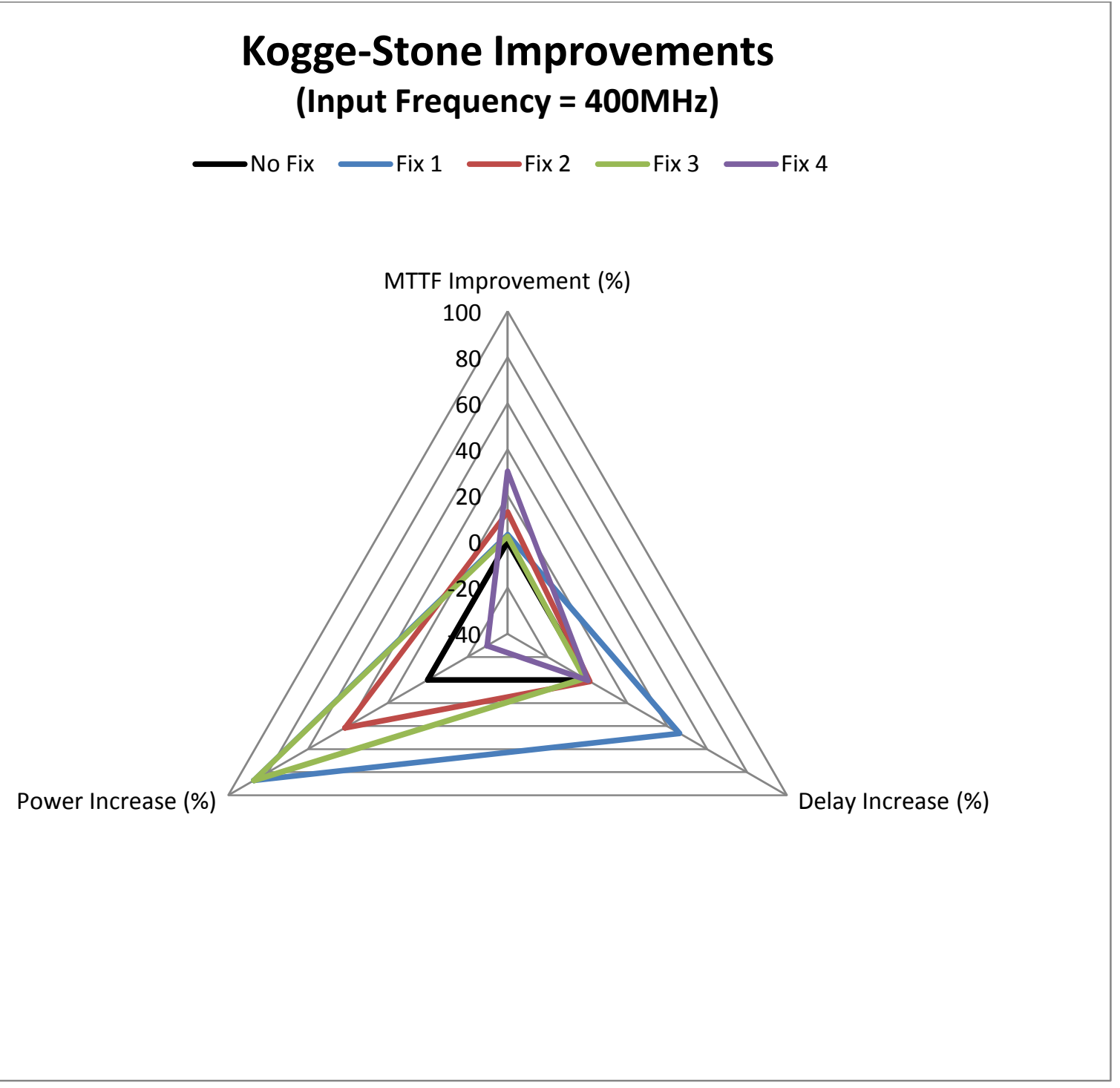

Figure 5.4: Results on Kogge-Stone Adder for Various Fixes @ 400MHz 


\begin{tabular}{|c|c|c|c|c|}
\hline Fix & Description & $\begin{array}{c}\text { MTTF } \\
\text { Increase }(\%)\end{array}$ & \begin{tabular}{|c|} 
Power \\
Increase $(\%)$
\end{tabular} & $\begin{array}{c}\text { Delay } \\
\text { Increase }(\%)\end{array}$ \\
\hline & \multirow{2}{*}{$\begin{array}{l}\text { AND and XOR gates } \\
\text { doubled in size, prefix logic } \\
\text { reduced to half its original } \\
\text { size, source/drain metal }\end{array}$} & 1.70 & 97.00 & 40.96 \\
\hline 1 & & 3.12 & 87.20 & 46.25 \\
\hline & \multirow{2}{*}{$\begin{array}{l}\text { Doubled source/drain metal } \\
\text { width, kept PPA blocks } \\
\text { original size, decreased the } \\
\text { size of the XOR and AND }\end{array}$} & 9.90 & 46.19 & 0.54 \\
\hline & & 12.98 & 41.46 & 1.29 \\
\hline & \multirow{2}{*}{$\begin{array}{l}\text { Doubled source/drain metal } \\
\text { width, increased the size of } \\
\text { the PPA blocks, XOR and } \\
\text { AND gates by } 30 \%\end{array}$} & 1.59 & 97.18 & -4.57 \\
\hline & & 2.40 & 87.12 & -1.39 \\
\hline & \multirow{2}{*}{$\begin{array}{l}\text { Doubled source/drain metal } \\
\text { width, decreased the size of } \\
\text { the weakest XOR gate by } \\
30 \%\end{array}$} & 24.26 & -37.06 & 0.21 \\
\hline & & 30.71 & -29.71 & 0.31 \\
\hline
\end{tabular}

Table 5.1: Comparison of Kogge-Stone fixes

(Top rows for each fix are for input frequency of $25 \mathrm{MHz}$ and bottom rows are for $400 \mathrm{MHz}$.

Fix 4 is most effective at increasing lifetime and decreasing power consumption with only a slight increase in delay. It focuses more on the weak link and doesn't require increased gate sizing which can increase power and delay. The weak link remained the same for the first three fixes, yet for fix 4, decreasing the gate size of the weak link XOR allows for a large MTTF improvement)

As you can see from table 5.1, FIX4 proved to be the most effective. The MTTF for both input frequencies showed the greatest increase, and the circuit even uses less power without sacrificing delay. The other fixes are also beneficial to the device, yet they don't have the same amount of improvement on MTTF and either slow the circuit down or use much more power. For purposes of this thesis, I really only care about prolonging the lifetime of the circuit, and at a minimum, doing so without drastically affecting performance. The increased metal sizing does require more area, but this is the single most effective way to significantly lower current density and reduce the likelihood of electromigration. 


\subsubsection{Register File}

The Register file was comprised of three different subcircuits: the MUXs, the decoders, the dynamic memory cells. I focused on three areas of the register file, specifically the MUXs, the decoders, and the inverter before the decoder output. Two potential fixes were tried, and the overall changes were similar to those done for the Kogge Stone adder. FIX 1 involved doubling the source/drain metal widths only. This allowed for a large reduction in current density yielding an increase in MTTF. Power and delay remain very much the same. FIX 2 involved increasing the metal lines and scaling down the gates for the MUXs and DECODERs by 25\%. MTTF increases, and there is a large reduction in power consumption with only a small increase in delay. Figures 5.6 and 5.7 show the improvements on MTTF for both fixes on the MUXs and Decoders.

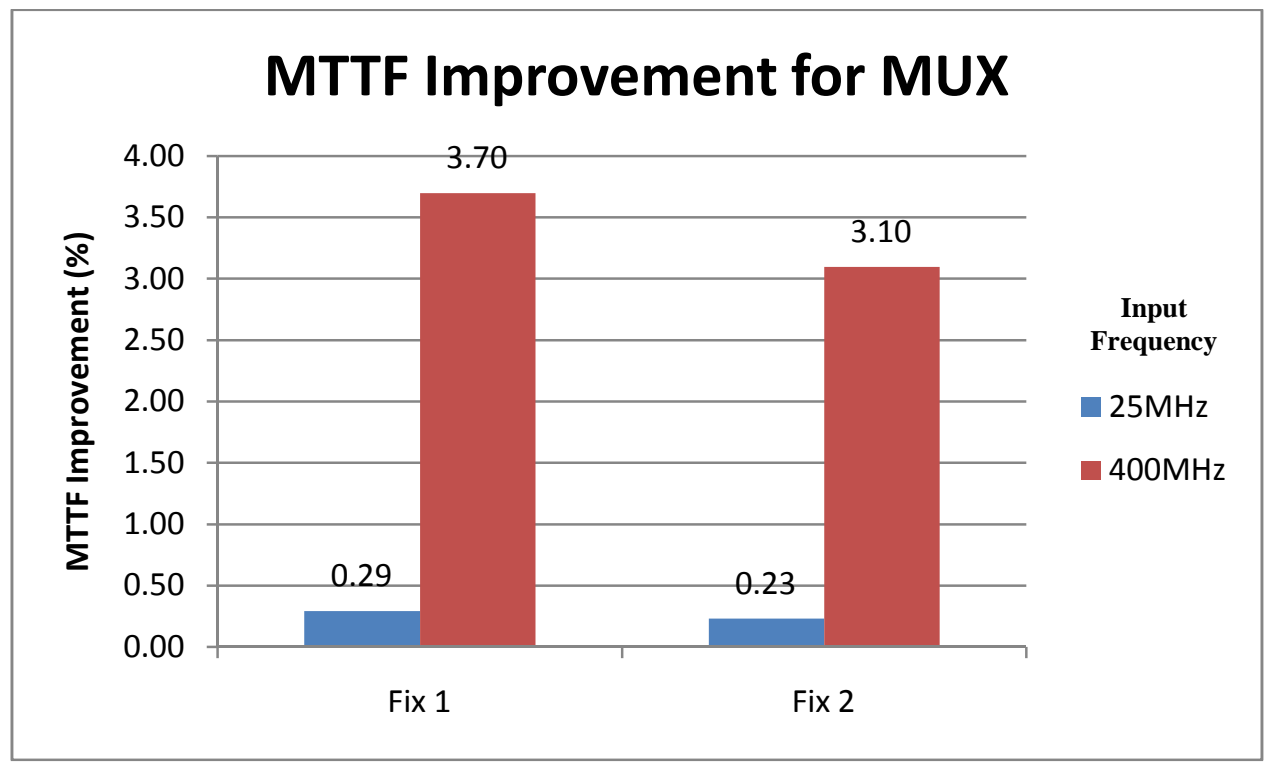

Figure 5.5: MTTF Results on Register File MUX for Various Fixes 


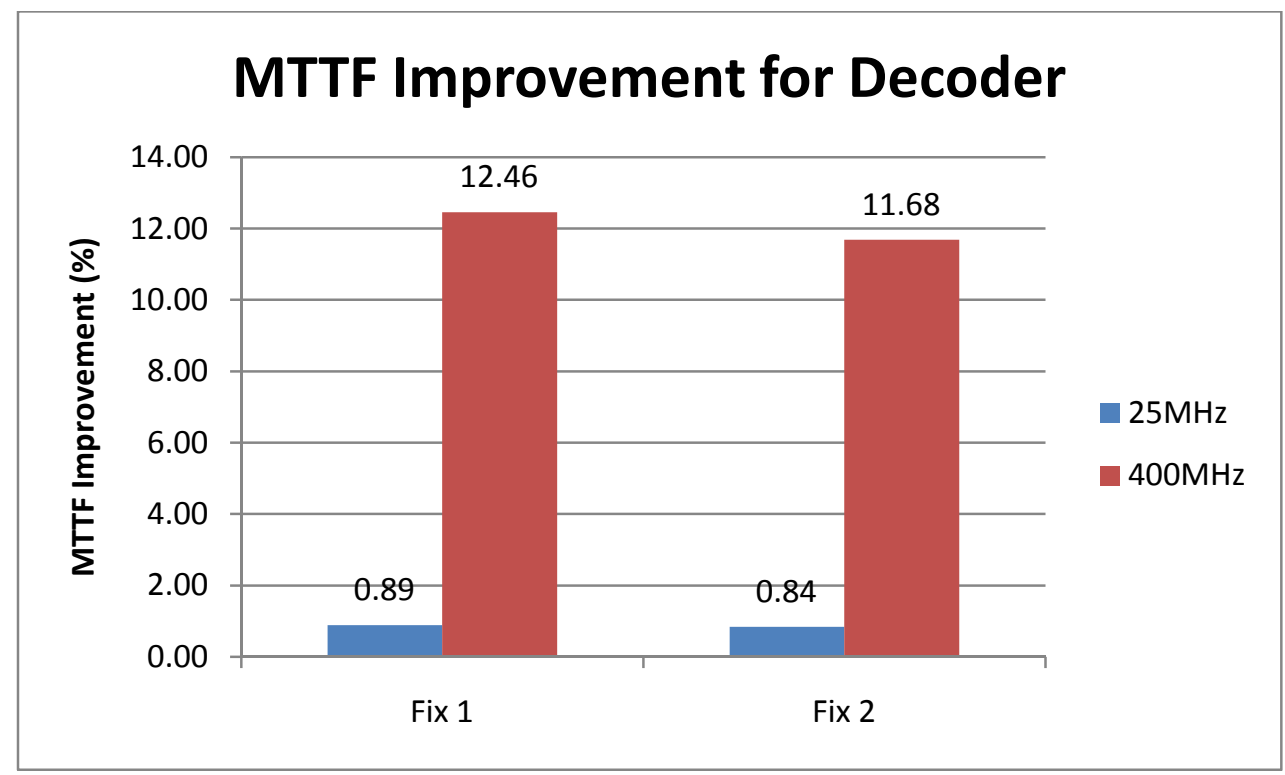

Figure 5.6: MTTF Results on Register File Decoder for Various Fixes

For both subcircuits, the fixes are more noticeable at faster input frequencies. MTTF improvement is roughly the same for both fixes, so both fixes will be applied to the entire register file. Figures 5.8 and 5.9 show the overall performance for the register file. 


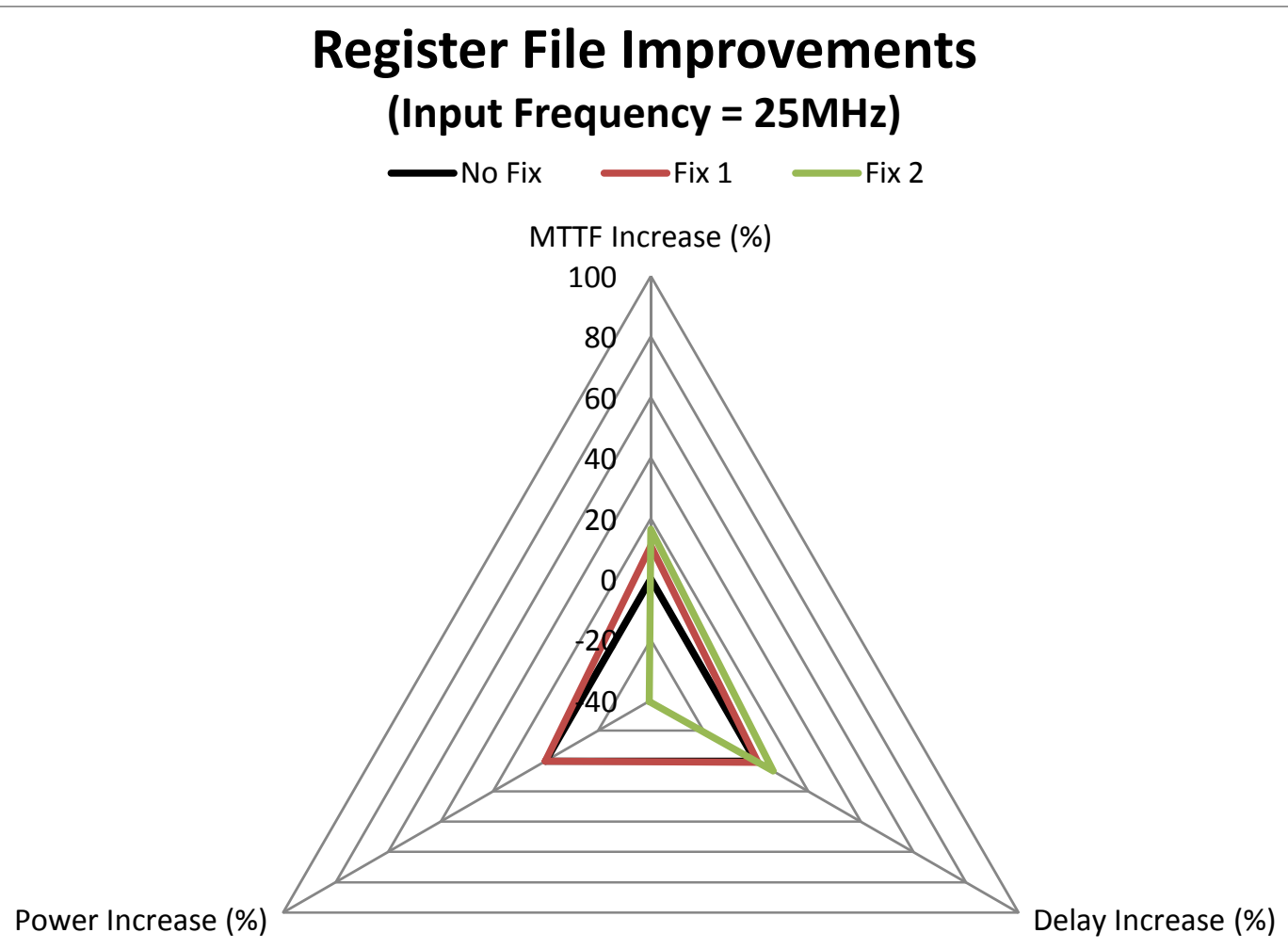

Figure 5.7: Results on Register File for Various Fixes @ 25MHz 


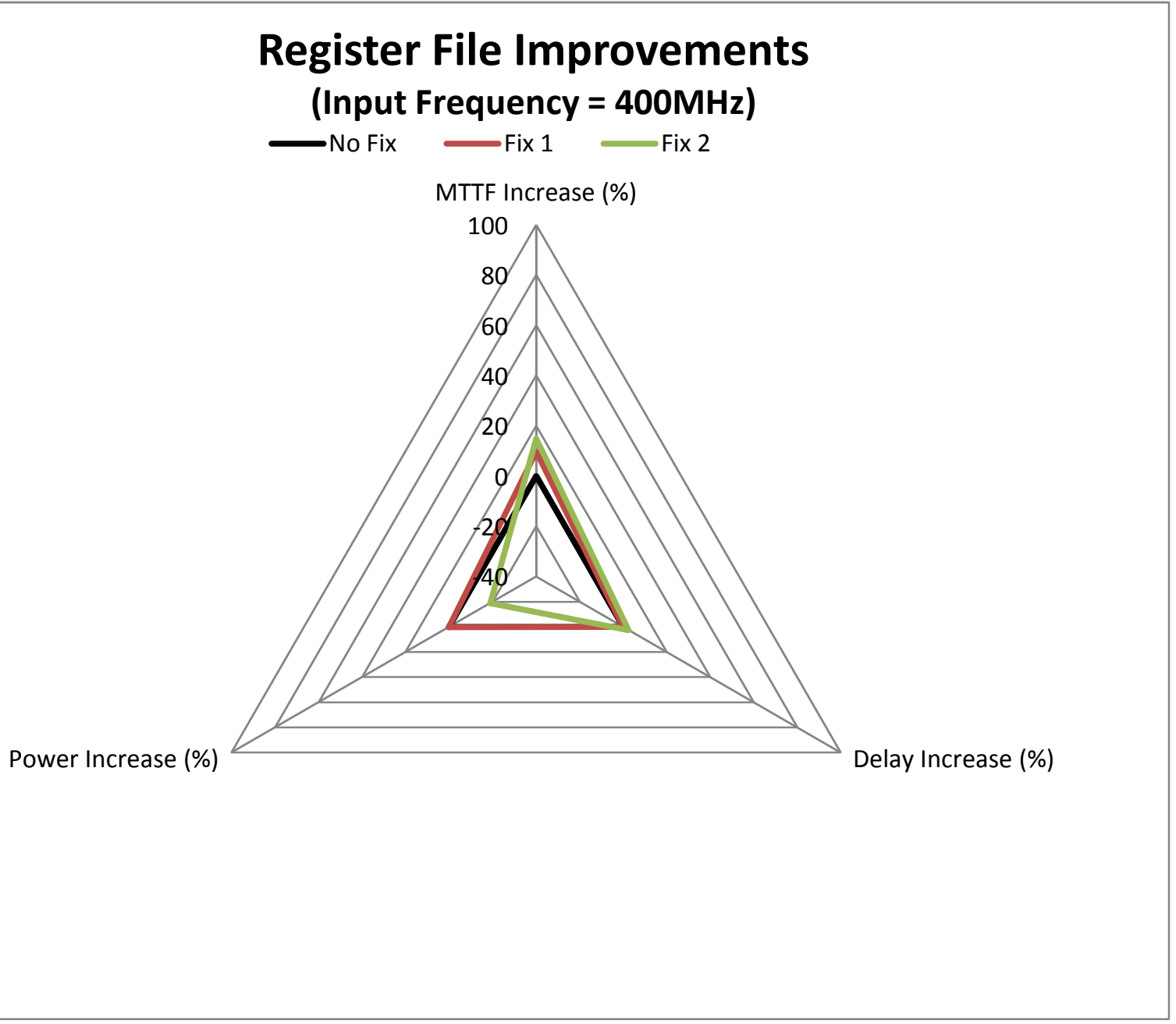

Figure 5.8: Results on Register File for Various Fixes @ 400MHz

As can be seen from the table 5.2, FIX 2 has the greatest impact on MTTF, increasing the overall lifetime over $15 \%$. As with the Kogge Stone adder, the reduced gate size consumes less power, about $30 \%$ less. The delay of the circuit remains roughly the same. There is a slight increase in delay with FIX 2, but it is only about a $6 \%$ increase. This is well worth the large power reduction and prolonged lifetime of the device. Again, the most effective way to improve MTTF is to increase interconnect sizing, reducing current crowding inside the metal lines. 


\begin{tabular}{|c|c|c|c|c|}
\hline Fix & Description & $\begin{array}{c}\text { MTTF } \\
\text { Increase }(\%)\end{array}$ & \begin{tabular}{c|} 
Power \\
Increase $(\%)$
\end{tabular} & $\begin{array}{c}\text { Delay } \\
\text { Increase }(\%)\end{array}$ \\
\hline \multirow[b]{2}{*}{1} & \multirow[b]{2}{*}{$\begin{array}{l}\text { Doubling the source/drain } \\
\text { metal line widths only }\end{array}$} & 11.04 & 0.85 & 0.12 \\
\hline & & 9.70 & 0.22 & 0.00 \\
\hline \multirow{2}{*}{\multicolumn{2}{|c|}{$\begin{array}{l}\text { Doubling source/drain metal } \\
\text { width and scaling down the } \\
\text { gates for the MUXs and } \\
\text { DECODERs by } 25 \%\end{array}$}} & 16.41 & -39.36 & 6.60 \\
\hline & & 14.91 & -18.97 & 2.54 \\
\hline
\end{tabular}

Table 5.2: Comparison of Register File Fixes

(Top rows for each fix are for input frequency of $25 \mathrm{MHz}$ and bottom rows are for $400 \mathrm{MHz}$.

Fix 2 is most effective here. Although the smaller MUX/Decoder gate sizes

Slow down the circuit slightly, they consume much less power and overall lifetime

Of the register file is significantly increased) 


\section{Chapter 6. Conclusions}

Electromigration will be a large problem in semiconductor devices if not addressed soon. With the ongoing scaling down process of microprocessors and other complex devices, the issue is becoming more and more of a reality. This paper shows the development of a tool chain that makes it possible to identify the mean-time to failure (MTTF) of several common and high priority circuits such as complex adders and memories. This tool chain allows designers to isolate weak-points in these circuits to improve the overall MTTF of the circuit. The result is that with a few simple changes, circuits can be redesigned to increase the MTTF, at minimal cost to the system. The tool chain is modular enough to update it with different MTTF models and therefore this is a platform that can be used in the future as EM becomes well understood.

With the current scaling down process, power consumption and temperature can drastically increase, as will current density. This causes an overflow of electron crowding in the metal lines connecting to source and drain locations of MOSFETS, and ultimately destroys the metal lines, causing the device to fail. Using the developed tool chain, these weak links can be pinpointed and a variety of simple and smart modifications to any circuit can lead to a drastic increase in Mean Time to Failure. Some of these potential fixes were explained in chapter 5, and included decreasing gate widths, increasing metal line widths, and in some cases, increasing gate size of driving stages. Designers must be more and more careful and keep these modification techniques in mind to ensure a greater lifetime for these devices. Unfortunately, this is only one time of wear-out seen in semiconductor devices, and although there are ways to address it, there are other potential areas of wear-out that can also decrease device lifetime. Luckily for me, the changes involved in increasing MTTF caused very little or no decrease in performance and only a slight increase if any in power consumption. Many times the power consumption was also 
reduced. In summary, one can see that just like there are design methods for maximizing speed and minimizing delay, there are also those to maximize circuit lifetime. that can improve MTTF; just as there are many ways to design a circuit for a specific purpose, there are also many ways to design a circuit for a maximum MTTF.

In completing this thesis, I have learned that there is no simple way to increase failure time. However, with the aid of the tool chain, weak links can be found and simple modifications can be made to maximize MTTF. There are certainly many design considerations one must consider, and if the discussed techniques are kept in mind, then circuit lifetime can definitely increase. However, as semiconductors continue to grow more complex and reduce in size, these techniques become harder to implement. Eventually, designers must be willing to sacrifice some performance in order to really see a great improvement in the lifetime of a device. 


\section{Chapter 7. Works Cited}

[1] Hauschildt, M., M. Gall, and S. Thrasher. "Analysis of Electromigration Statistics for $\mathrm{Cu}$ Interconnects." Applied Physics Letters 88 (2006).

[2] Hiroyuki, Abe, Sasagawa Kazuhiko, and Saka Masumi. "Electromigration Failure of Metal Lines." International Journal of Fracture (2006): 219-240.

[3] Computer Simulation Laboratory. 2005.

<http://www.csl.mete.metu.edu.tr/Electromigration/emig.htm>.

[4] Yamanaka, Kimihiro, Yutaka Tsukada, and Katsuaki Suganuma. "Solder Electromigration in $\mathrm{Cu} / \mathrm{in} / \mathrm{Cu}$ Flip Chip Joint System." Journal of Alloys and Compounds 437 (2007): 186-190.

[5] Gras, R., and L.G. Gosset. "Integration and Characterization of Gas Cluster Processing." Microelectronic Engineering 84 (2007): 2675-2680.

[6] Tang, Pin F. "Simulation and Computer Models for Electromigration." Electromigration and Electronic Device Degradation. John Wiley \& Sons, Inc, 1994. 27-77.

[7] Zeghbroeck, B. Van. "Chapter 7: MOS Field-Effect-Transistors." Principles of Semiconductor Devices. 2004. <http://ecewww.colorado.edu/ bart/book/book/chapter7/ch7_7.htm>.

[8] Subramoniam, R. A Statistical Model of Oxide Breakdown Based on a Physical Description of Wearout. Proceedings of IEEE International Electron Devices Meeting, 13 Dec. 1992, Electron Devices Soc. IEEE.

[9] Pecht, Michael, and Pradeep Lall. "Temperature Dependencies on Electromigration." Electromigration and Electronic Device Degradation. John Wiley \& Sons, Inc, 1994. 79-104.

[10] Hu, C.-K., L. Gignac, and R. Rosenberg. "Electromigration of Cu/Low Dielectric Constant Interconnects." Microelectronics Reliability 46 (2006): 213-231.

[11] Lee, J.C., Chen Ih-Chin, and Hu Chenming. "Modeling and Characterization of Gate Oxide Reliability." IEEE Transactions on Electron Devices 35 (1998).

[12] Henderson, Christopher L. "Time Dependent Dielectric Breakdown." Semiconductor Reliability. 2002. 〈http://www.semitracks.com/manuals/12.pdf>.

[13] Allers, K.-H. "Prediction of Dielectric Reliability From I-V Characteristics: Poole-Frenkel Conduction Mechanism Leading to Root(E) Model for Silicon Nitride MIM Capacitor." Microelectronics Reliability 44 (2003): 411-423.

[14] Black, J.R. "Electromigration-a Brief Survey and Some Recent Results." IEEE Transactions on Electron Devices (1969). 
[15] Black, J.R. "Metallization Failures in Integrated Circuits." RADC Technical Report (1968).

[16] Kirchheim, R., and U. Kaeber. "Atomistic and Computer Modeling of Metallization Failure of Integrated Circuits By Electromigration." Journal of Applied Physics 70 (1991): 172-181.

[17] Noguchi, Junji. "Dominant Factors in TDDB Degradation of Cu Interconnects." IEEE TRANSACTIONS ON ELECTRON DEVICES, 52 (2005): 1743-1750.

[18] Shiono, N, and M Itsumi. "A Lifetime Projection Method Using Series Model and Acceleration Factors for TDDB Failures of Thin Gate Oxides." 31st Annual Proceedings. Reliability Physics 1993 (1993): 1-6.

[19] Rodder, M. "Oxide Thickness Dependence of Inverter Delay and Device Reliability for 0.25um CMOS Technology." International Electron Devices Meeting 1993. Technical Digest (1993).

[20] Chen, Chih, and S. W. Liang. "Electromigration Issues in Lead-Free Solder Joints." J Mater Sci: Mater Electron (2007).

[21] Cheng, Y.l., Y.1. Wang, and H.C. Chen. "Effect of Inter-Level Dielectrics on Electromigration in Damascene." Thin Solid Films 494 (2006): 315-319.

[22] Zhao, Jian H. "Theoretical and Experimental Study of Electromigration." Electromigration and Electronic Device Degradation. John Wiley \& Sons, Inc, 1994. 167-233.

[23] Blech, I.A. "Electromigration in Thin Aluminum Films on Titanium Nitride." Journal of Applied Physics 47 (1976): 1203-1208.

[24] Sankaranarayanan, Karthik. "[Hotspot] Multiple Core Chip Thermal Model." The HotSpot Archives. 4 Feb. 2008. <http://www.cs.virginia.edu/pipermail/hotspot/>.

[25] Wu, W., S. H. Kang, J. S. Yuan, and A. S. Oates. "Electromigration Performance for $\mathrm{AI} / \mathrm{SiO} 2, \mathrm{Cu} / \mathrm{Si} 02$ and Cdow-K Interconnect Systems including Joule Heating Effect." School of Electrical Engineering \& Computer Science, University of Central Florida, Orlando, FL 32816. Print.

[26] Wu, W., S. H. Kang, J. S. Yuan, and A. S. Oates. "Thermal effect on electromigration performance for $\mathrm{Al} / \mathrm{SiO} 2, \mathrm{Cu} / \mathrm{SiO} 2$ and $\mathrm{Cu} / \mathrm{low}-\mathrm{K}$ interconnect systems." Chip Design and Reliability Laboratory, School of Electrical Engineering and Computer Science, University of Central Florida, P.O. Box 162450, Orlando, FL 32816-2450, USA. Print.

[27] Black, J. R. Electromigration. Wikipedia. Web. 07 Aug. 2007. $<$ http://en.wikipedia.org/wiki/Electromigration>. 
[28] Johnson, R. W., J. R. Broomstead, and G. B. Weir. "200 C operation of semiconductor power devices."Components, Hybrids, and Manufacturing Technology, IEEE Transactions on 16.7 (1993): 759-64. Print. 


\section{Appendices}

\section{Appendix A: Background Information}

\section{Layout Process}

To familiarize you with the formal layout process, below is an image showing the layout of a 2 input XOR gate.

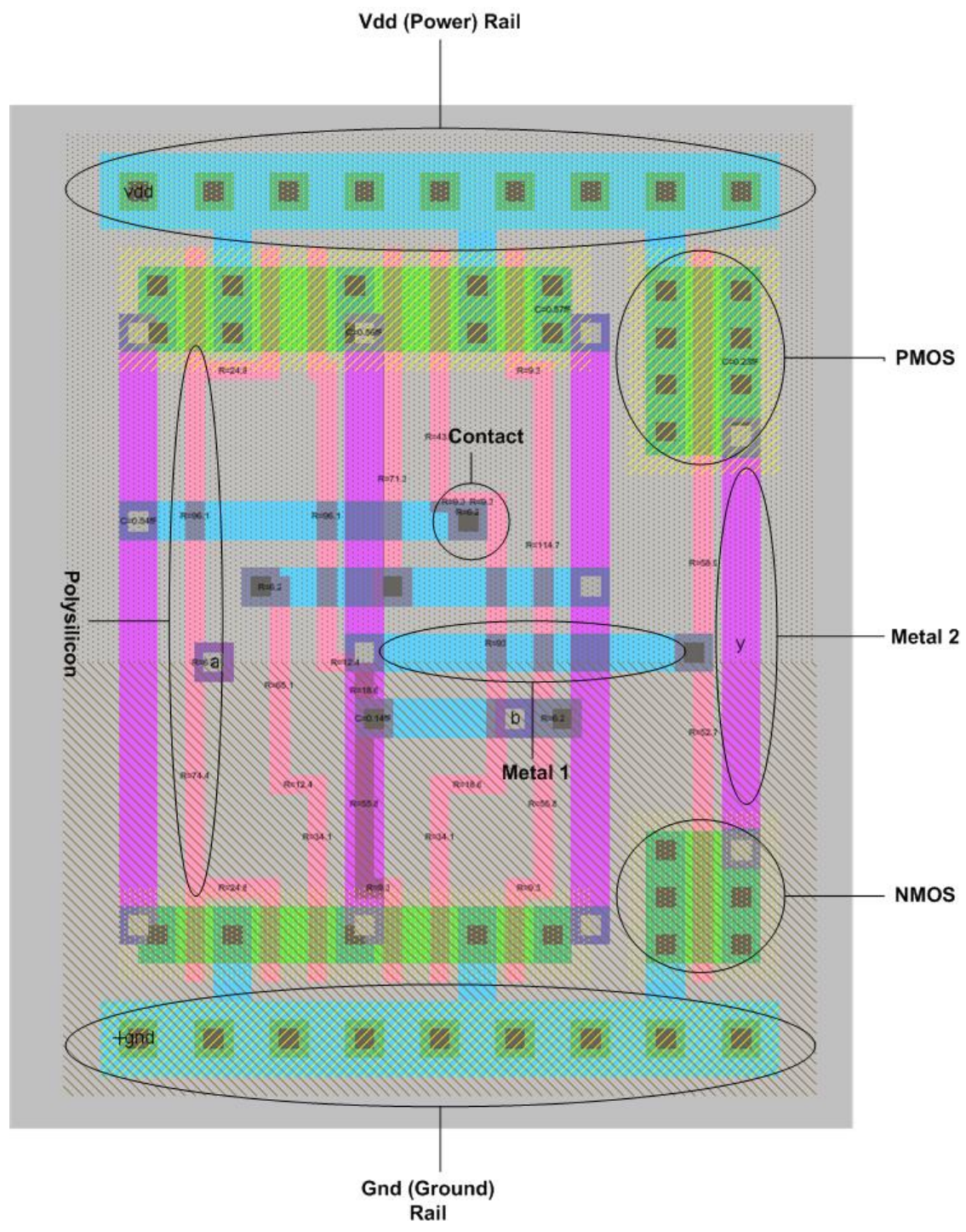

Figure A.1: Basic Layout of 2-input XOR gate, showing key components. 
Above is the layout of a 2-input XOR gate. Now, the layouts can be done in a variety of ways, but you always want to design with practicality in mind. You can see the PMOS and NMOS transistors circles and the silicon that connects any of the gates between them all. The top rail is my power (vdd) and the bottom is ground (gnd). The blue metal represents metal layer 1 and the purple metal represents metal layer 2. The various small "squares" are contacts, used for connecting different metal types or materials together. You can see both the inputs "a" and "b" as well as the output " $y$ " appear as contacts. The light green region show what's known as "diffusion", or the source and drain for each of the FETs.

This is obviously not a project on how to layout circuits, however, it is important to discuss a few issues. When laying out any design, you always want keep transistors, contacts, metals, etc as closely together as possible, obviously to minimize space. You must however follow the design rules specified by the layout preferences you set up from the beginning. Following design rules closely helps minimize the amount of parasitic. It is also best to use as few metal layers as possible, because with them come lots of parasitic capacitances and resistances as well. Parasitic capacitance, in electric circuits, is the unavoidable and usually unwanted capacitance that exists between the parts of an electric component or circuit simply because of their proximity to each other [27]. Parasitic capacitance can also exist between closely space conductors, such as metal lines, or PCB traces. The parasitic resistance of a metal or polysilicon line can also have a profound influence on the signal propagation delay over that line. The resistance of a line depends on the type of material used (polysilicon, aluminum, gold, copper, etc), the dimensions of the line and finally, the number and locations of the contacts on that line (http://siwww.epfl.ch/LSI2001/teaching/webcourse/ch04/ch04.html). 
Layout Diagrams for Small Circuits

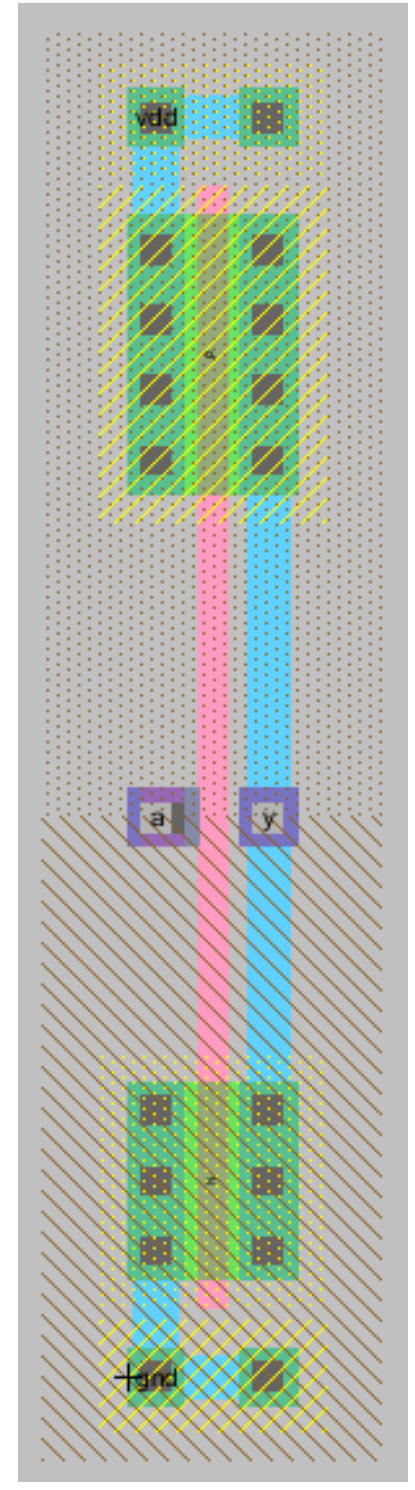

Figure A.2: Inverter with S/D Metal Line Width of $75 \mathrm{~nm}$

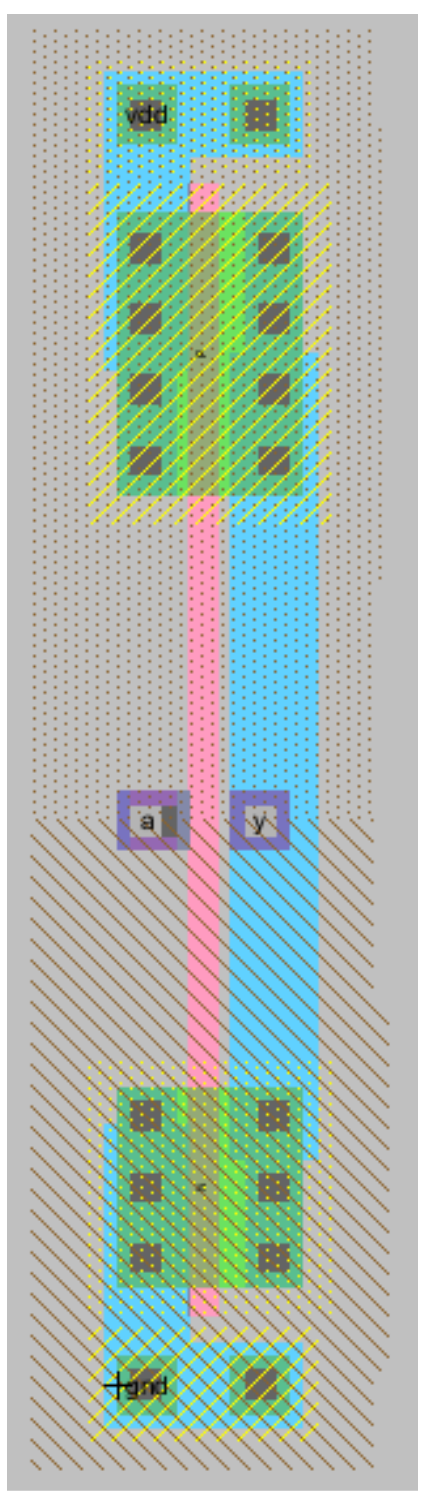

Figure A.3: Inverter with S/D Metal Line Width of $150 \mathrm{~nm}$ 


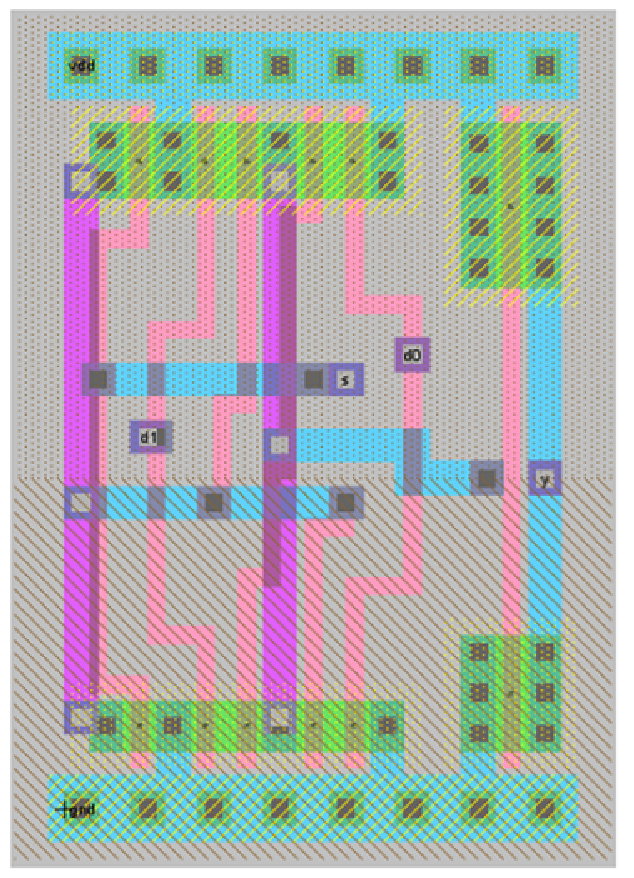

Figure A.4: The layout for a 2-1 MUX

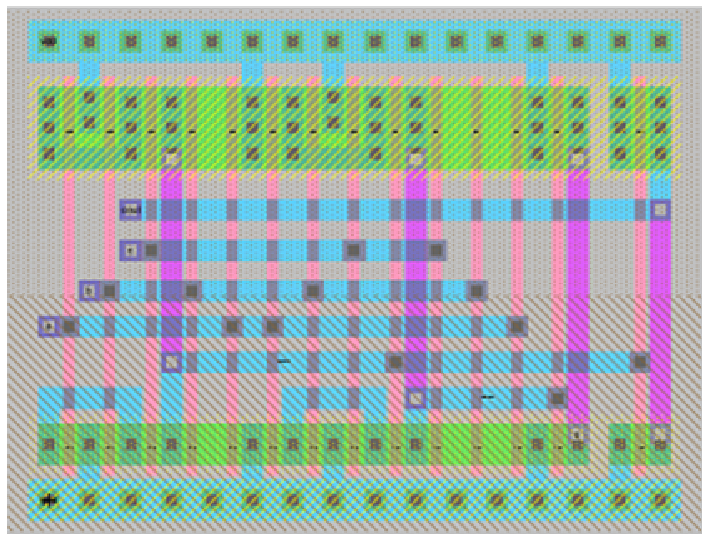

Figure A.5: The layout for a Full Adder

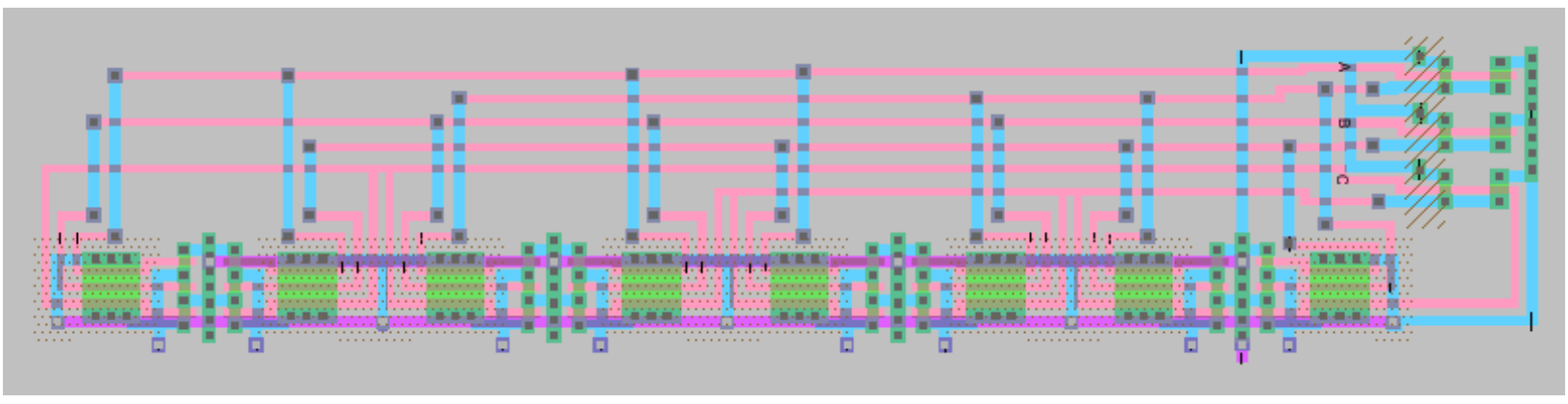

Figure A.6: Layout of the 3-to-8 Decoder. 


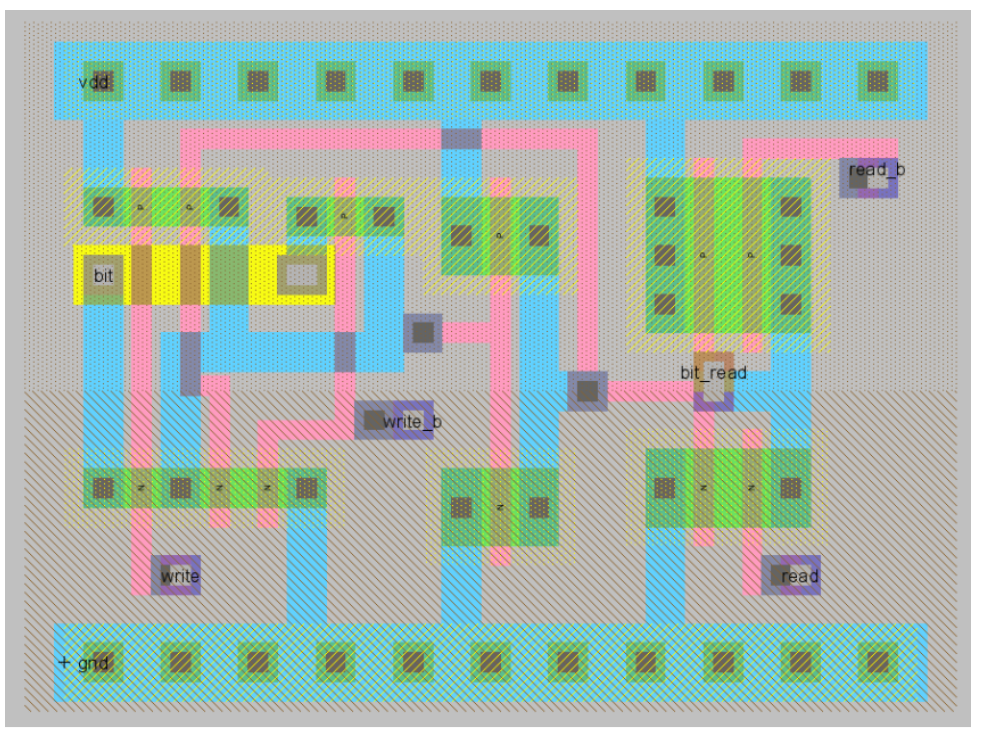

Figure A.7: Layout of a 12 Transistor SRAM Cell

\section{Circuit Diagrams for Small Circuits}

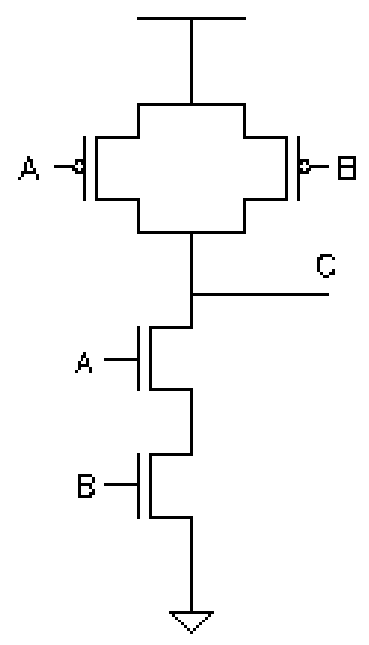

NAND

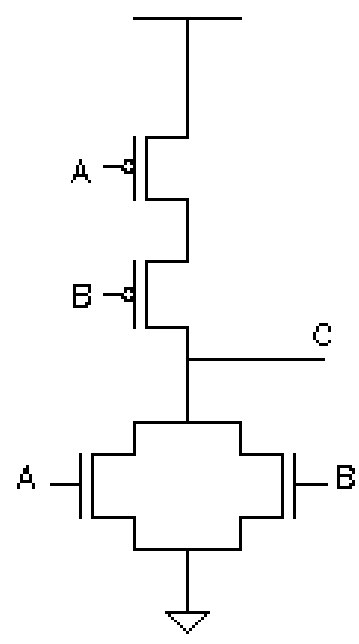

$\mathrm{NOF}$

Figure A.8: The Circuit for a NAND-gate (left), and a NOR-gate (right). 


\begin{tabular}{|cc|c|}
\hline$A$ & $B$ & $A \oplus B$ \\
\hline 0 & 0 & 0 \\
0 & 1 & 1 \\
1 & 0 & 1 \\
1 & 1 & 0 \\
\hline
\end{tabular}

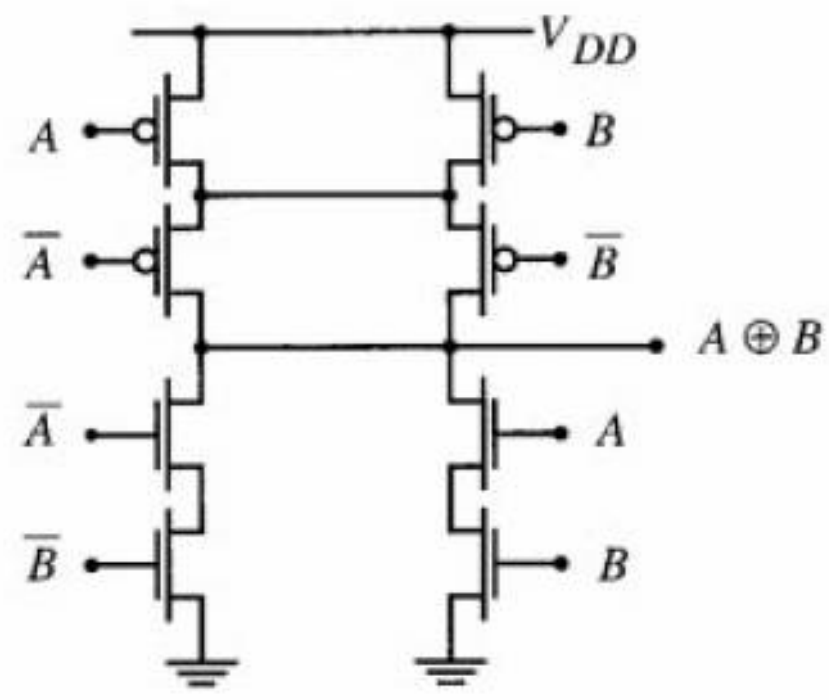

Figure A.9: Transistor Network that Creates an Exclusive-OR function.

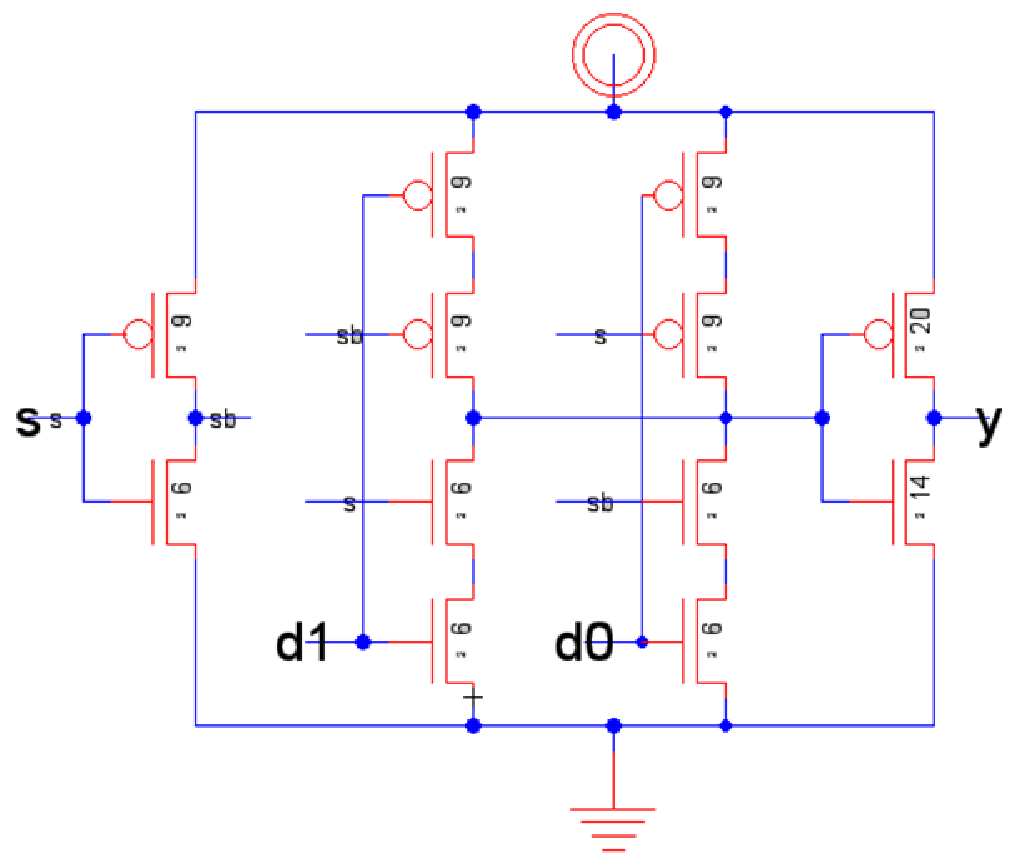

Figure A.10: 2-1 MUX Circuit Diagram 


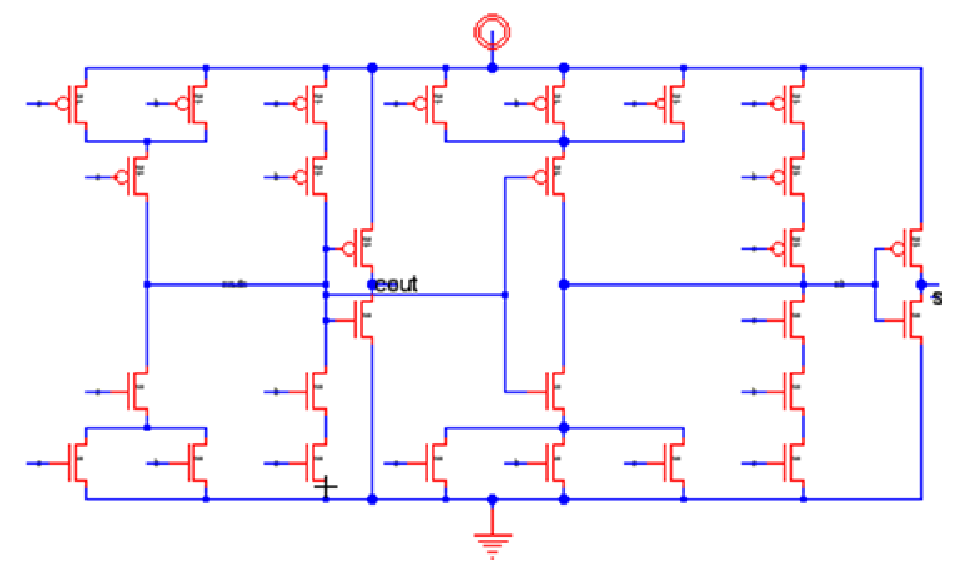

Figure A.11: Full Adder Circuit Diagram

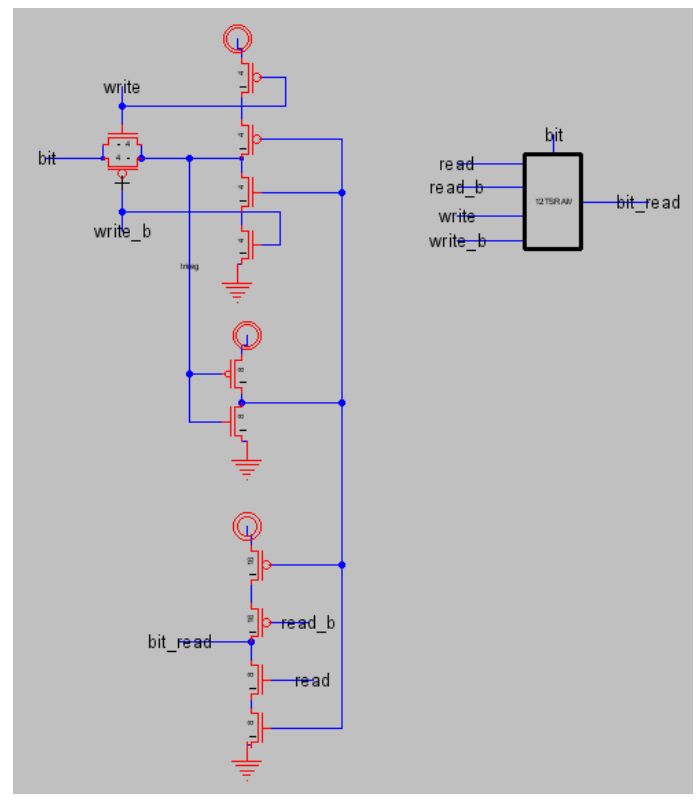

Figure A.12: 12 Transistor SRAM Circuit Diagram

\section{CMOS Kogge-Stone}

The propagate and generate gate circuits are shown below for even layer and odd layer tiles. Each layer produces inverted outputs from negating logic gates. The circuits shown below are shown is CMOS, whereas my signals were created using Output Prediction Logic (OPL). This is a simple tweak and simply requires the addition of a precharge transistor between VDD and Vout and a footer transistor at the bottom of the NMOS stack to ground. 
One other issue with the Kogge-Stone is the unbalanced loading of individual tiles. Tiles in the top right corner of the tree are required to drive more gates than tiles in the left of the tree. This should require sizing some of the gates with higher output capacitance up to keep delay through the tree fairly even, however with the current OPL design this sizing adjustment was not made and I assume that the gates will drive their load adequately.
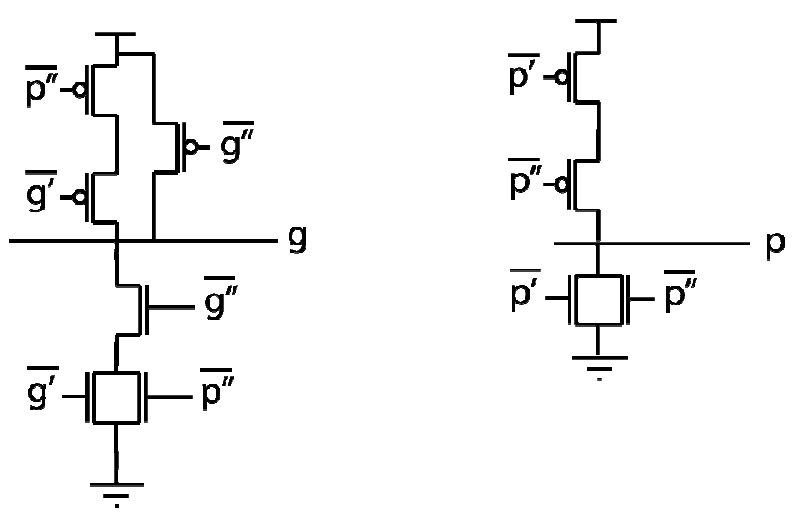

Figure A.13: Even layer tiled CMOS PPA circuit (inverted inputs, non-inverted outputs)

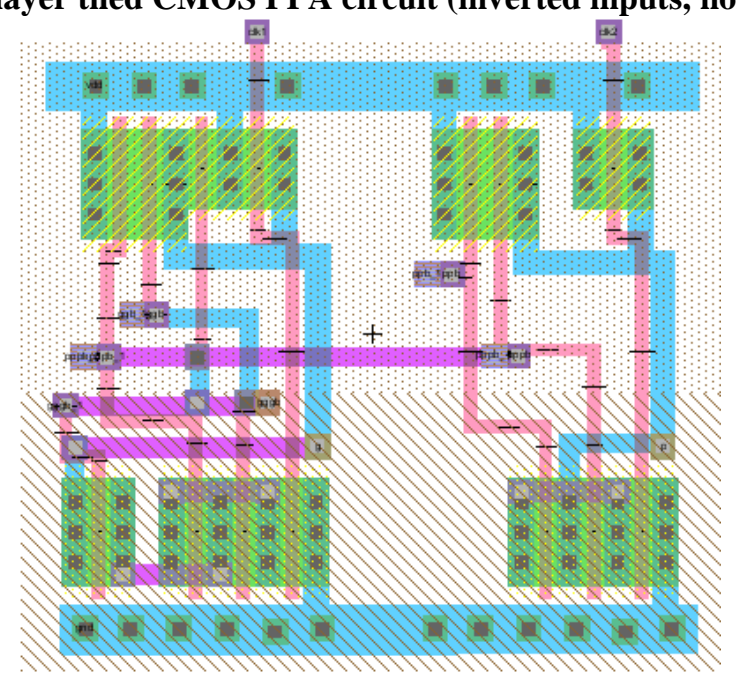

Figure A.14: Even layer tiled CMOS-OPL PPA circuit layout cell 

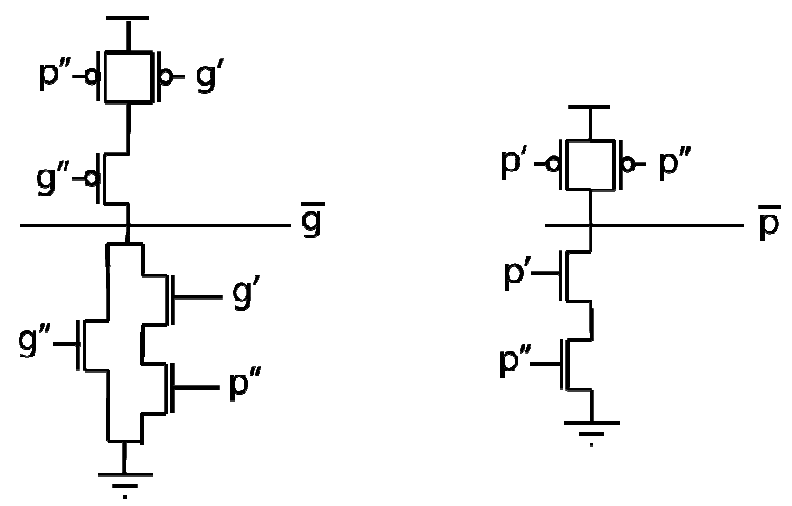

Figure A.15: Odd Layer Tiled CMOS PPA circuit (non-inverted inputs, inverted outputs)

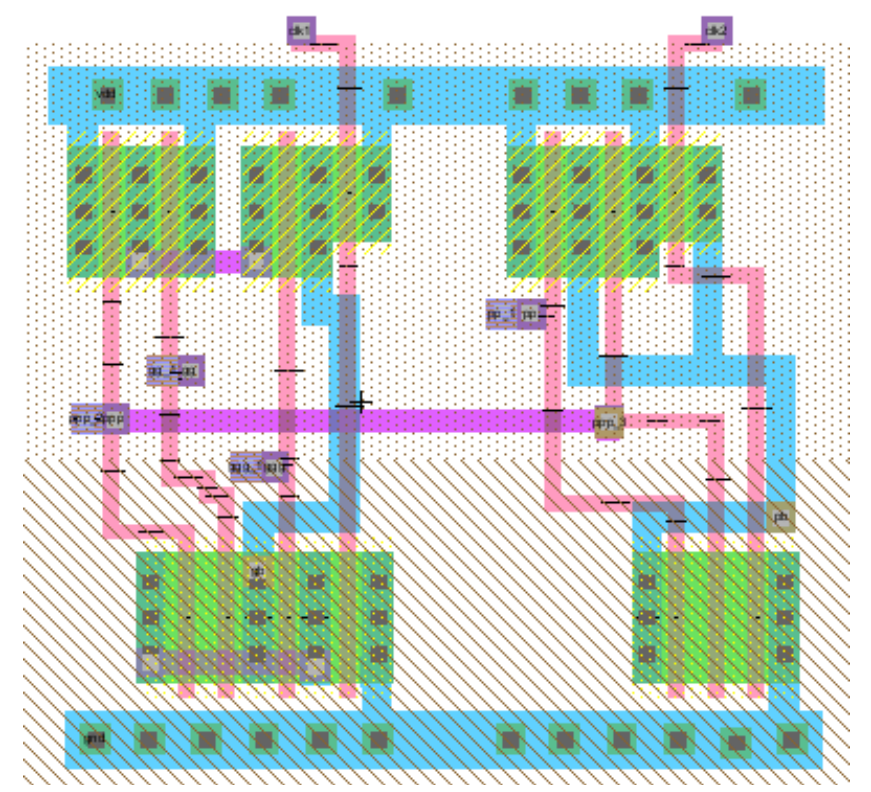

Figure A.16: Odd Layer Tiled CMOS-OPL PPA circuit cell

The architecture selected for the final design is OPL Kogge-Stone Radix-2 Prefix Adder. This adder was chosen because the PPA sub-circuits already developed and tested would properly evaluate the carry-in values for each bit in a Kogge-Stone prefix tree. The PPAs of the K-S tree generate the same carry-in for each bit that the tiles of the CLA would generate, and therefore both adder architectures require similar front and back logic blocks. The front logic block takes $\mathrm{Xi}$ and $\mathrm{Yi}$ (inputs) and in 2 logic stages outputs the propagate (pi) and generate (gi) signals for each bit. Those signals are inputs to the K-S tree, along with the carry-in for bit 0 , and the 
outputs of the K-S tree are the carry-in (ci) for each bit. The K-S tree has five logic stages. The final two stages calculate the sum (Si) for each bit and the carry-out (C16) for the adder. The basic design requires nine logic stages from $\mathrm{Xi}$, Yi to $\mathrm{Si}$ and $\mathrm{C} 16$.
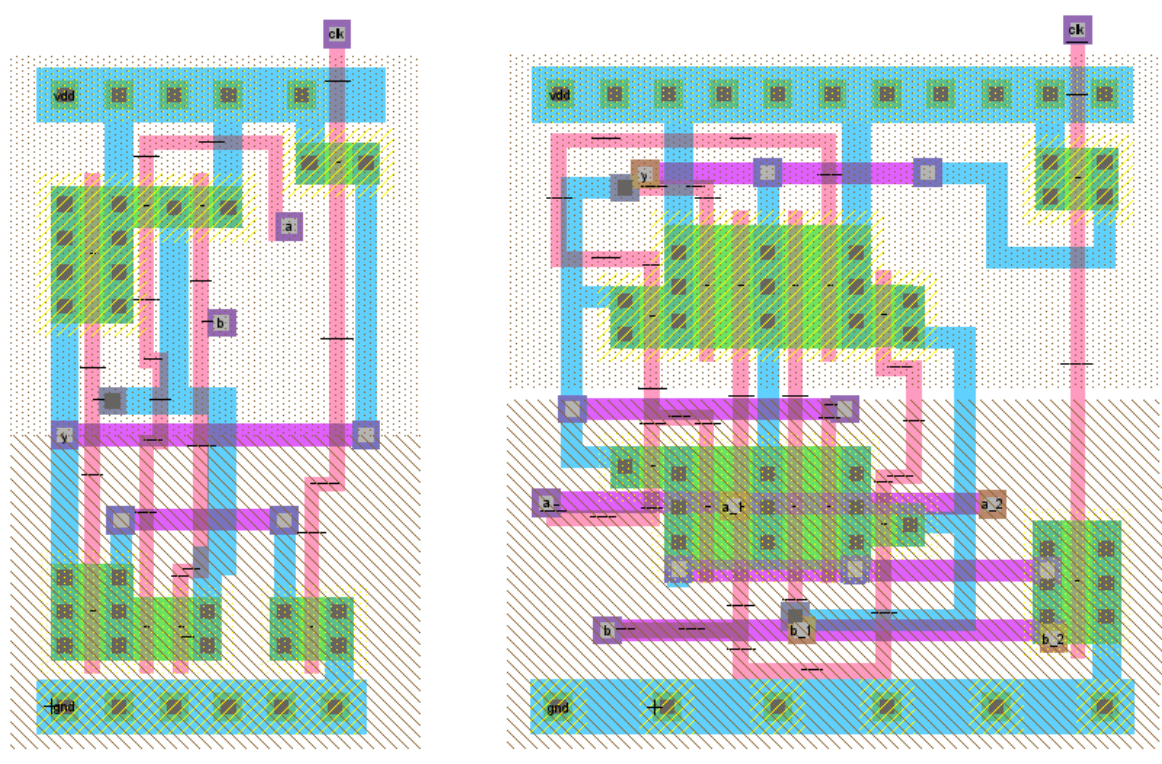

Figure A.17: CMOS OPL layout circuits for AND (left) and XOR (right) gates used for the Pi and Gi signals The complete 16-bit Kogge-Stone Parallel Prefix Adder layout is shown below. The center of the layout area has three north-south parallel signal lines for VDD, GND, and the clock (CLK). These branch at each layer of the tree to provide connection to the individual cells. The last PPA for evaluating carry-out from the Gi/Pi bit 15 column has been pushed up into the fourth row to save total area in the cell; that fourth row is shifted to the right by one PPA cell to accommodate that last row collapse. 


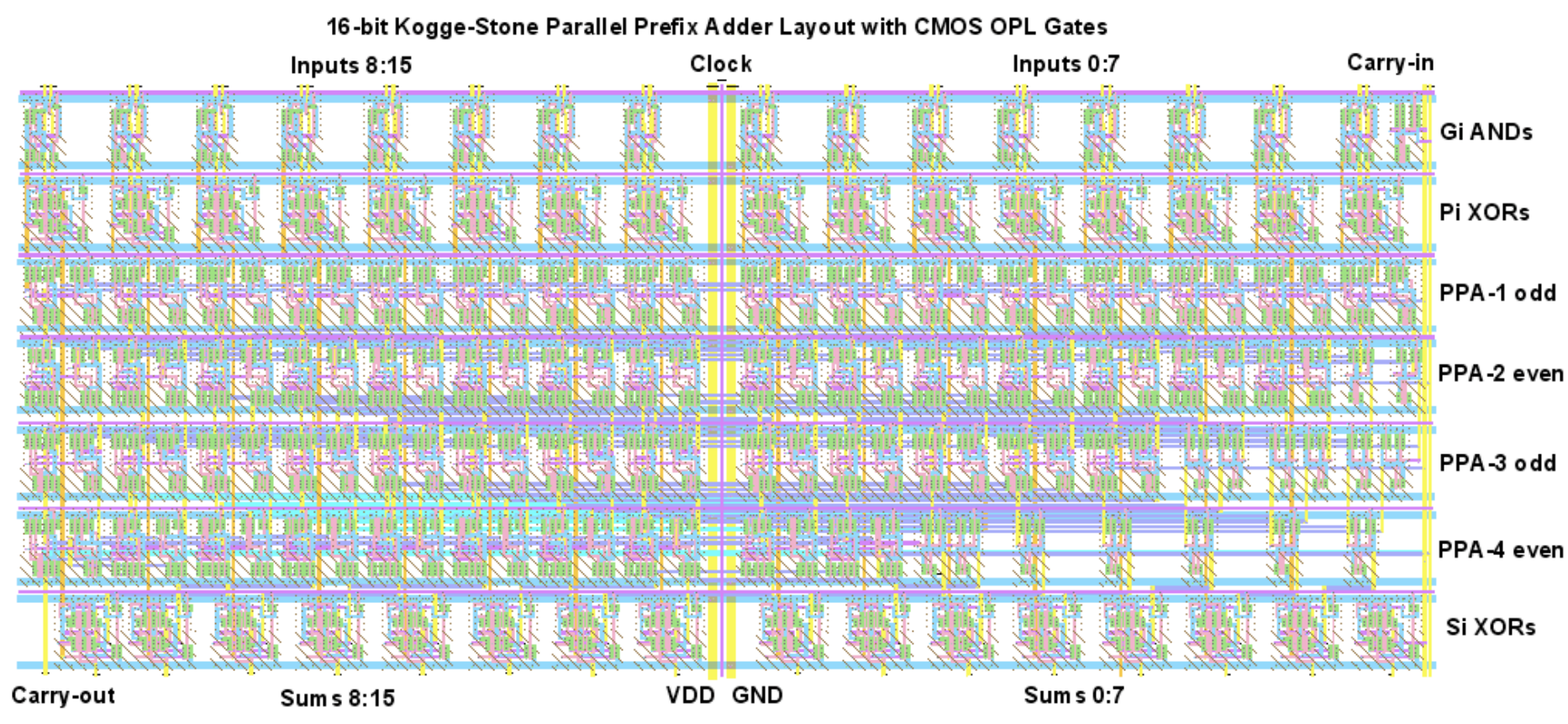

Figure A.18: Complete 16-bit Kogge-Stone Adder layout 


\section{Appendix B: Small Circuit Evaluation}

The following sections analyze failure times for small circuits. All tested gates assumed 2 ideal inputs, yielding all input-output combinations. I will call this input strategy "ideal inputs". Ideally, having an average gate voltage exactly half of the rail voltage over a long period of time ensures the gate is ON/OFF for equal time periods. This however doesn't mean that the failure locations will be at the gate. In fact, current densities at the gate are often much lower than source/drain locations. This input strategy will be referred to as the "optimal voltage" which also corresponds to the "optimal current density". The data obtained from gate-level simulations is very important, as these basic gates are the building blocks for subcircuits used in more complex, higher level designs.

\section{The NAND and NOR Gates}

The NAND and NOR gates have a very similar design. If given ideal inputs, their failure times are roughly the same. For the NAND gate, the higher current densities are found in the NMOS FETs, as all the current has a straight path to ground through both FETs. However, with the PMOS FETs, there is a current split between the two. The two gates are essentially "flipped" versions of one another so the behavior makes sense.

The temperature difference across the gate is too small to have any real effect on MTTF. Thus, it is the current density that readily determines the failure time. Still, the circuits are too small for the current density changes to have any drastic effect on MTTF. Also, just like the inverter, higher MTTF means more power. 


\section{The XOR-gate}

The XOR-gate is made using two inverters for the inputs and eight (8) MOSFETS arranged in the network shown in figure 7.9. With ideal inputs, all FETs are on $50 \%$ of the time, thus all locations can be said to have "optimal current density". Again, the source/drain locations are most prone to failure as this is where the majority of interconnect material is found, not at the gates (mostly silicon). I can also note that with faster inputs comes a much more erratic current and higher current density which in turn lowers the MTTF. Figures 7.19 and 7.20 shows the source current for NMOS 1 for input frequencies of $25 \mathrm{MHz}$ and $400 \mathrm{MHz}$. NMOS 1 is the one of FETs found at the pull down network (PDN) closest to GND and it makes sense as to why this would be a weak link. Here the PDN is very active and the current has a clear path from the source to ground. Current is much higher here, and this yields an increased current density. However, when the input frequency is $25 \mathrm{MHz}$ the source current is able to stabilize and stays minimal. At $400 \mathrm{MHz}$, the source current is very erratic and current spiking can be seen, which increases the average current. 


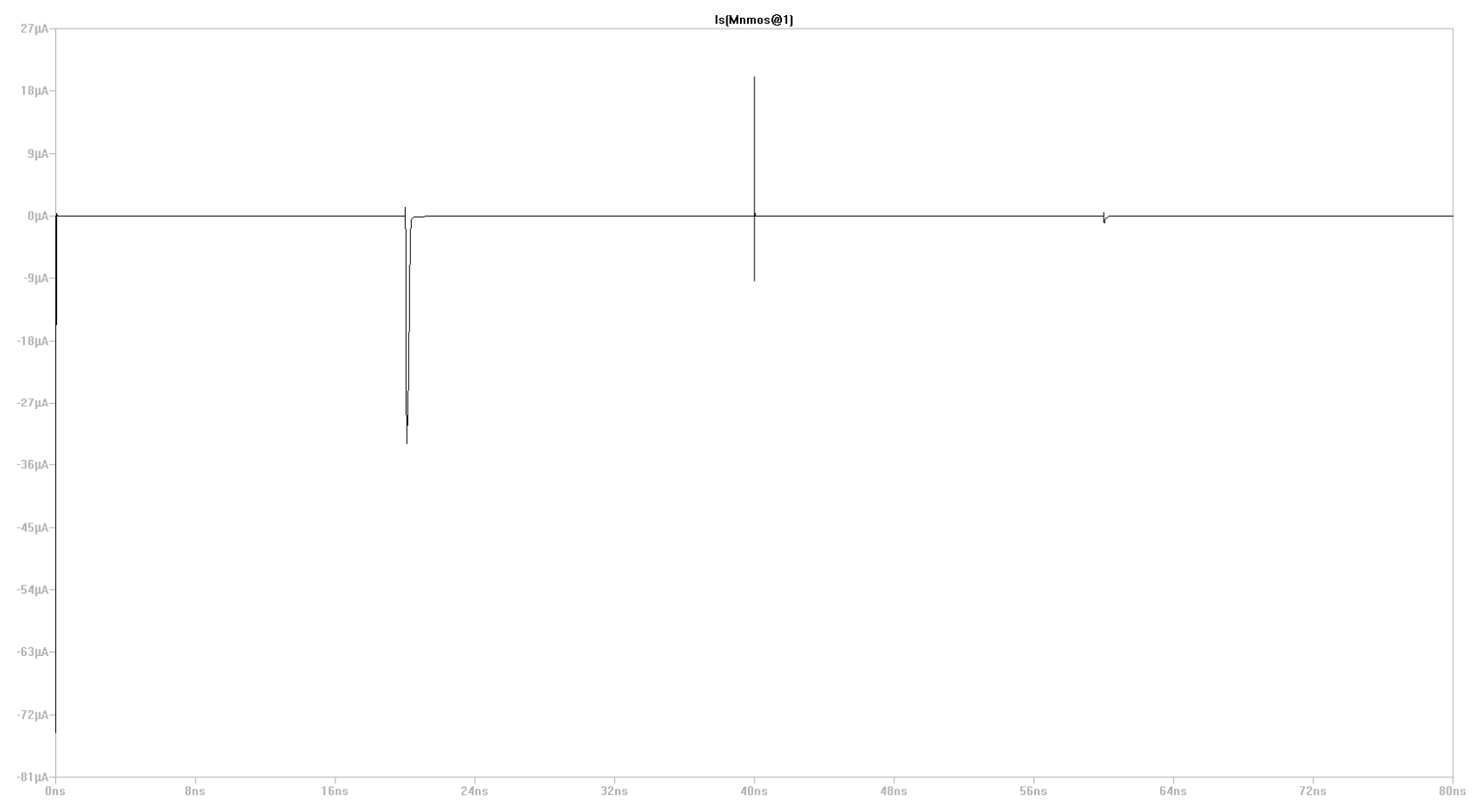

Figure B.1: NMOS1 Source Current for $25 \mathrm{MHz}$

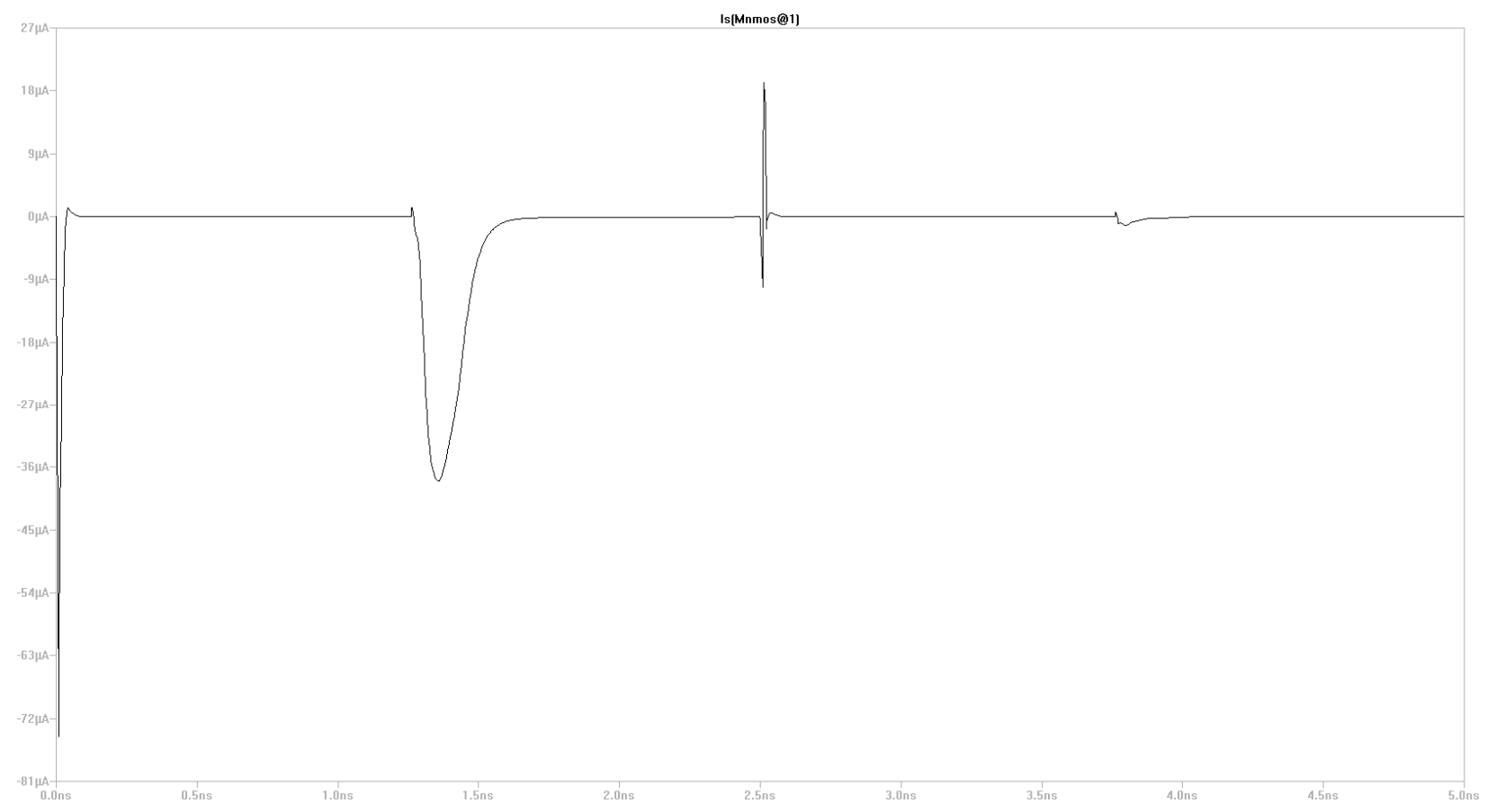

Figure B.2: NMOS1 Source Current for 400MHz 
For the case when the input frequency is $25 \mathrm{MHz}$, it was seen that the average source current of NMOS1 stays low, minimizing current density. The failure site for this case is actually at the source of PMOS5. Here the source is the power rail so naturally, source current and current density will be high. When the frequency reaches $400 \mathrm{MHz}$, the circuit is much less stable. A higher average current is found at the source of NMOS1 as the current takes significantly more time to stabilize.

\section{The AND and OR Gates}

The AND gate was designed using a NAND gate as a basic, and placing an inverter at the output. Similarly, the OR gate was designed using a NOR gate. For the AND gate, assuming ideal inputs, the failure location is at the source of NMOS1 despite the input frequency. For the OR gate, the failure location is the source of PMOS0 at $25 \mathrm{MHz}$ and the drain of PMOS1 at faster switching frequencies.

For the AND gate, NMOS1 is the top FET in the PDN. If I look at the voltage at the gate of this FET, it is high $75 \%$ of the time, meaning this FET is on $75 \%$ of the time. With more usage comes higher current density. Seeing as how it's on 3x longer than off, it makes sense that it fails first. The OR gate is a bit more interesting. At an input frequency of 25MHz, PMOS0 fails first. Current density is highest at the source of this FET, which happens to be the power rail as seen with the XOR gate. Any current leaving the power rail is going to be significant when compared to other locations, as the power rail is high $100 \%$ of the time. At higher frequencies, the failure site moves to PMOS1, where current density is highest at the drain. Similar to the AND gate, the voltage at the gate of PMOS1 is low $75 \%$ of the time, meaning this FET is on $75 \%$ of the time. Naturally, current density is much higher in this region, making it a prone site to failure. 
Obviously, at the higher frequencies the higher current densities move away from the power rail to other prone sites.

Just like the XOR-gate, increasing the input frequency affects the MTTF of the two gates. The faster switching frequencies prevent the logic levels from reaching full high/low potential and make the currents much more irregular. This increases the average current through a given location, yielding a higher current density thus a lower MTTF.

\section{Multiplexer (MUX)}

The MUX utilizes inverters but none of the standard gates and instead uses the transistors to effectively connect the output inverter to the rail or ground based on what the select bit is. By using the information gathered from my gate-level circuits, one can assume that one of the PMOS FETs is likely to fail first. First of all, all PMOS FETs are connected to the power rail and will naturally have high current densities through the interconnect material. After simulating the MUX, it turns out that the weak link is PMOS5. This is a very reasonable conclusion, as this is the PMOS of the large inverter before the output of the MUX. The CMOS logic before this stage has a heavy load to drive, not to mention that the size of the PMOS is much larger than any of the other FETS. Also, the CMOS stage has quite a bit of interconnect material and current through this stage is not forced into a particular area. At the inverter stage, all current from the power rail flows directly into PMOS5.

Second, the input to the inverter is stressed, and the rise/falls times are slow, preventing the output from reaching its full potential. Thus, this region is my failure site. Again, temperature was not a huge factor in determining MTTF for this device. 


\section{Full Adder}

The full adder was implemented using the following equations:

$$
\begin{gathered}
\mathrm{C}_{\text {out }}=\mathrm{C}(\mathrm{A}+\mathrm{B})+\mathrm{AB}, \\
\mathrm{S}=! \mathrm{A} ! \mathrm{BC}+! \mathrm{AB} ! \mathrm{C}+\mathrm{A} ! \mathrm{B} ! \mathrm{C}+\mathrm{ABC}
\end{gathered}
$$

The alternative to the sum would be:

\section{$\mathrm{S}=\mathrm{A}$ xor B xor $\mathrm{C}$}

Using the first set of equations reduces transistor count, as the sum is generated using part of the carry out logic. Similar to the MUX, no standard logic gates are used to implement the full adder. Inverters are used, but the rest of the design used standard FETs. Analyzing this circuit is also similar to what was done for the MUX.

The weak link for this design of the full adder is NMOS5, which is the NMOS shown in the middle of figure 7.5. NMOS5 is part of one of the inverters used in the design and is very heavily stressed. The input to this FET is /Cout, and seeing as determining the carry out bit requires a significant amount of logic this is a likely failure location. The output of this inverter is also the piece of carry logic used to obtain the sum. The drain of this FET happens to be /Sum, and adds additional stress to this region. The waveforms below show the drain/source current for this FET. Again, the glitches/current spiking bring the average source/drain current up significantly increasing current density. Temperature for the full adder is not really a factor in its failure. The next circuits all make use of subcircuits, and Hotspot will soon show that self heating becomes a much larger factor. 


\section{Decoder}

The 3-to-8 decoder used for this project consists of NOR-gates and inverters. There are three inverters that make up the inverter chain, which supplies the 3 inputs (A, B, and C) and their compliments. The appropriate inputs were then connected to one of the NOR-gates in the NORchain to obtain the correct logic. The weakest link for this design was the second furthest PMOS from the inverted B signal. Again, the PMOS FETs typically experience much larger current densities than the NMOS FETs, so this failure site is justifiable. However, the B signal is only the second fastest input, which means speed was not the main factor in determining failure. Temperature as well was not a huge factor here, as this was one of the cooler areas on the device. Ultimately, very high current density is what caused this FET to fail. This PMOS is in the middle of the 3 FET chain found in the NOR gate, and current density at the source is very high. It shares its source with the drain of the first PMOS in the chain, which has all the current fed from VDD. The results for this decoder are somewhat interesting, as the failing PMOS is on less than it is off. Also, average gate voltage is about $660 \mathrm{mV}$, higher than the nominal value of $500 \mathrm{mV}$. These are reasons as to why this wouldn't be the weakest link, but they do not outweigh the very high current running through the FET. Self heating is not yet a factor, but will for sure play a role in the more complex circuits.

\section{Summary}

In this section, it was shown that for small circuits, current density and input frequency are the two main factors in determining MTTF. Higher frequencies and current densities yield lower MTTFs. Temperature does play a role, however for these small circuits, temperature is roughly the same throughout the device. The differences have negligible effect on MTTF. Typically, FETs that are on longer than they are off are prone failure sites. The failure locations 
are different in each case, depending on the concentration of current density in each area. In theory, given ideal inputs, all FETs should be on/off for equal amounts of time. However, it is clear that this is not always the case. 


\section{Appendix C: Results for Small Circuits}

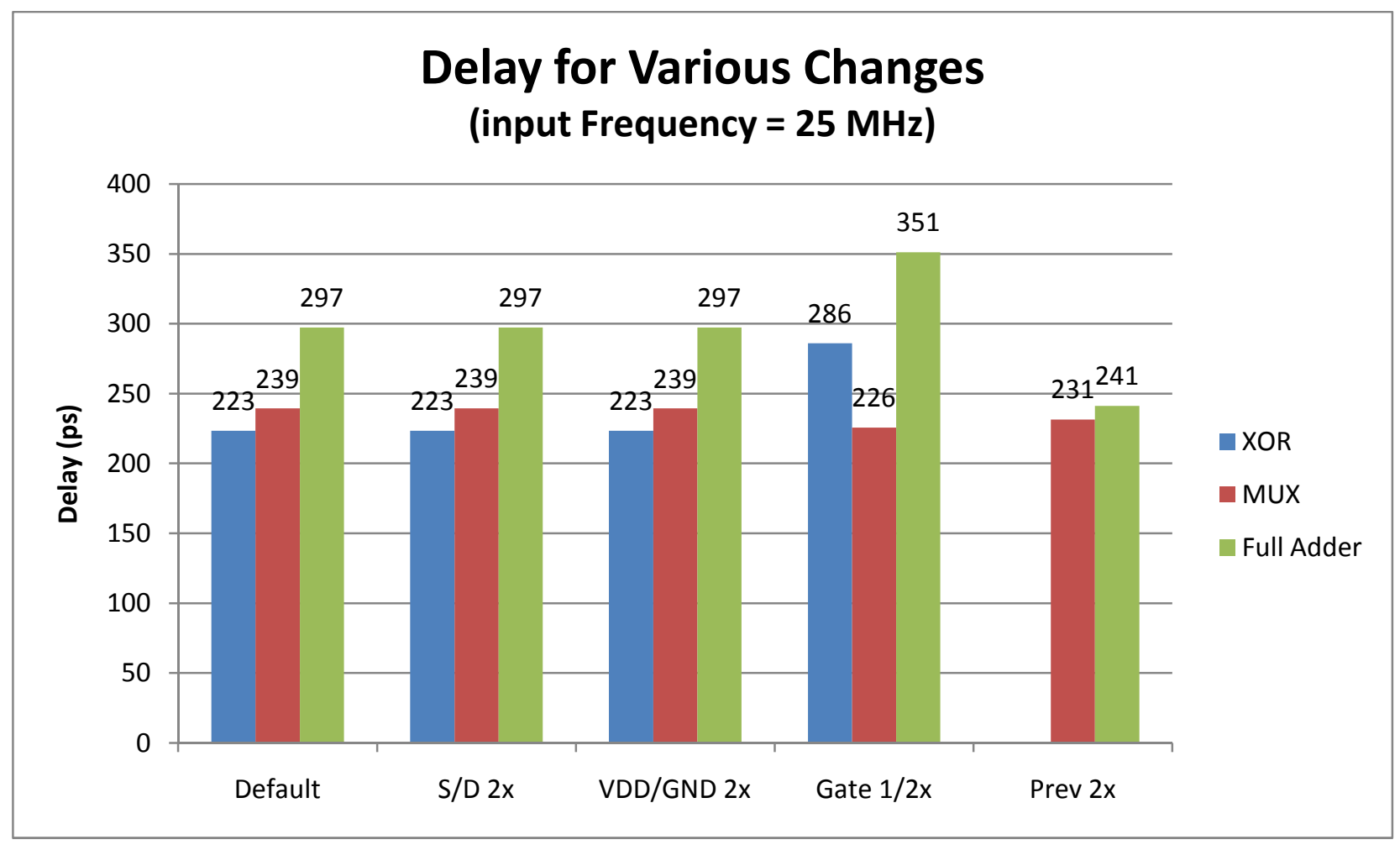

Figure C.1: Delay for Various Changes to Small Circuits @ $25 \mathrm{MHz}$ 


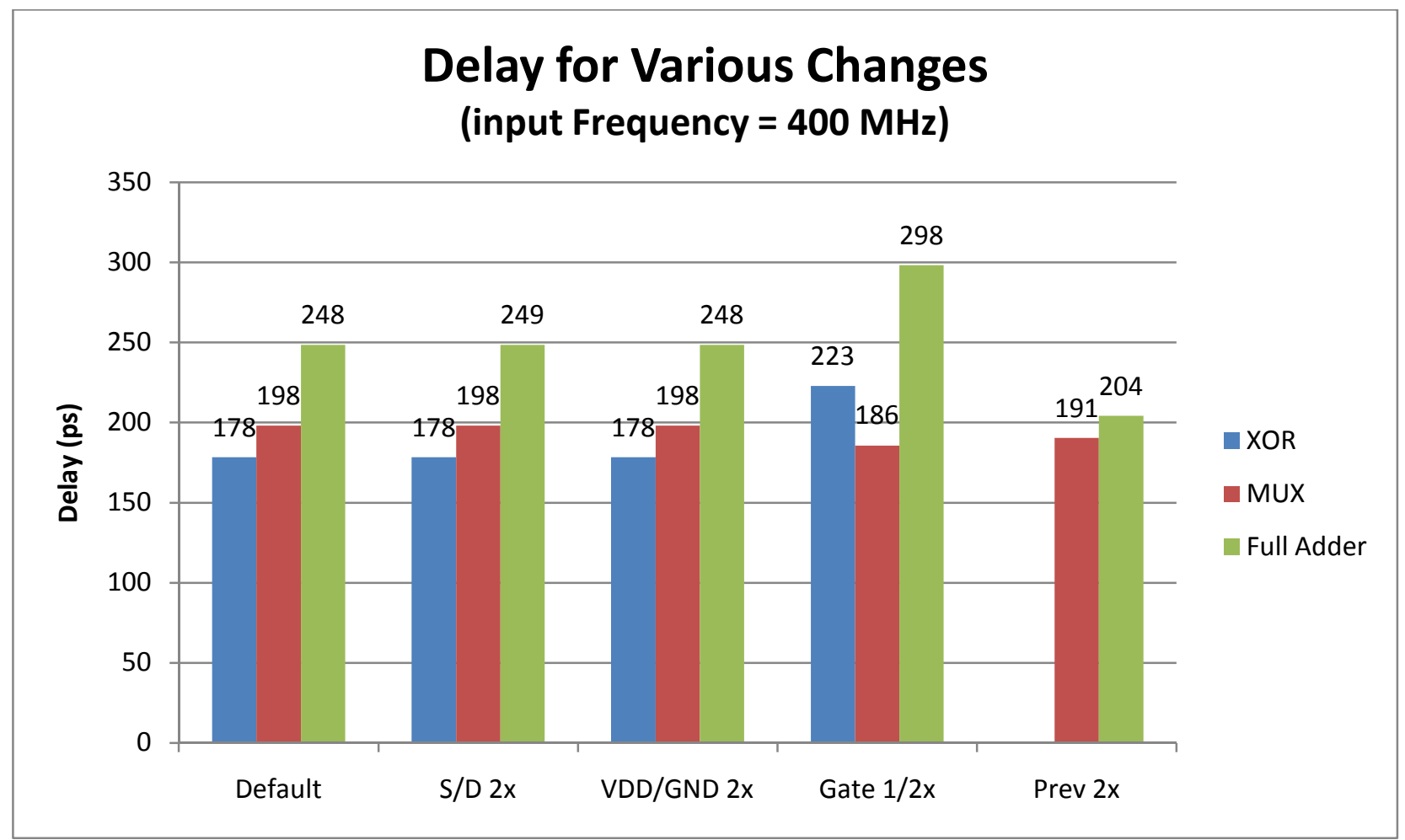

Figure C.2: Delay for Various Changes to Small Circuits @ 400 MH

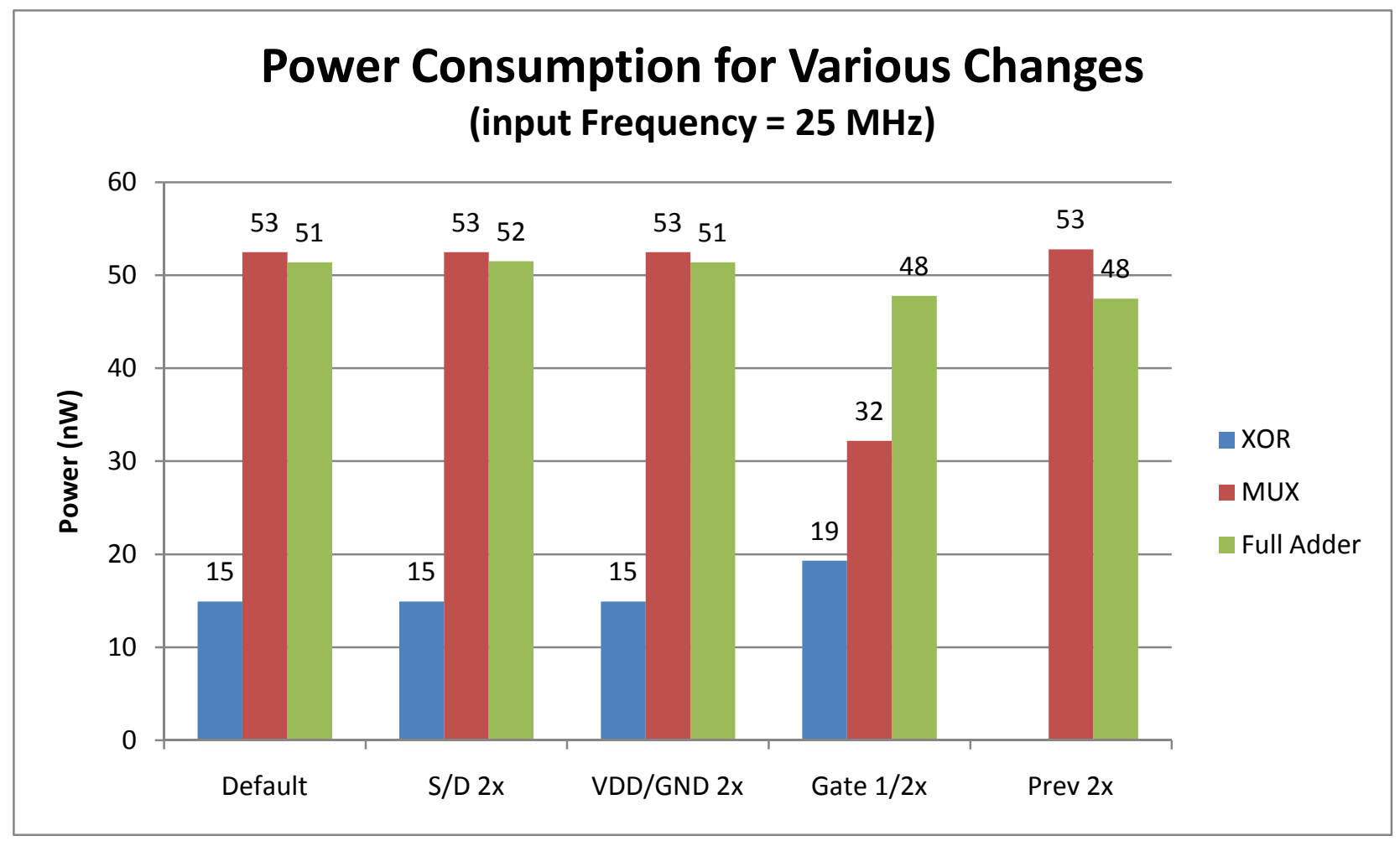

Figure C.3: Power Consumption for Various Changes to Small Circuits at $25 \mathrm{MHz}$ 


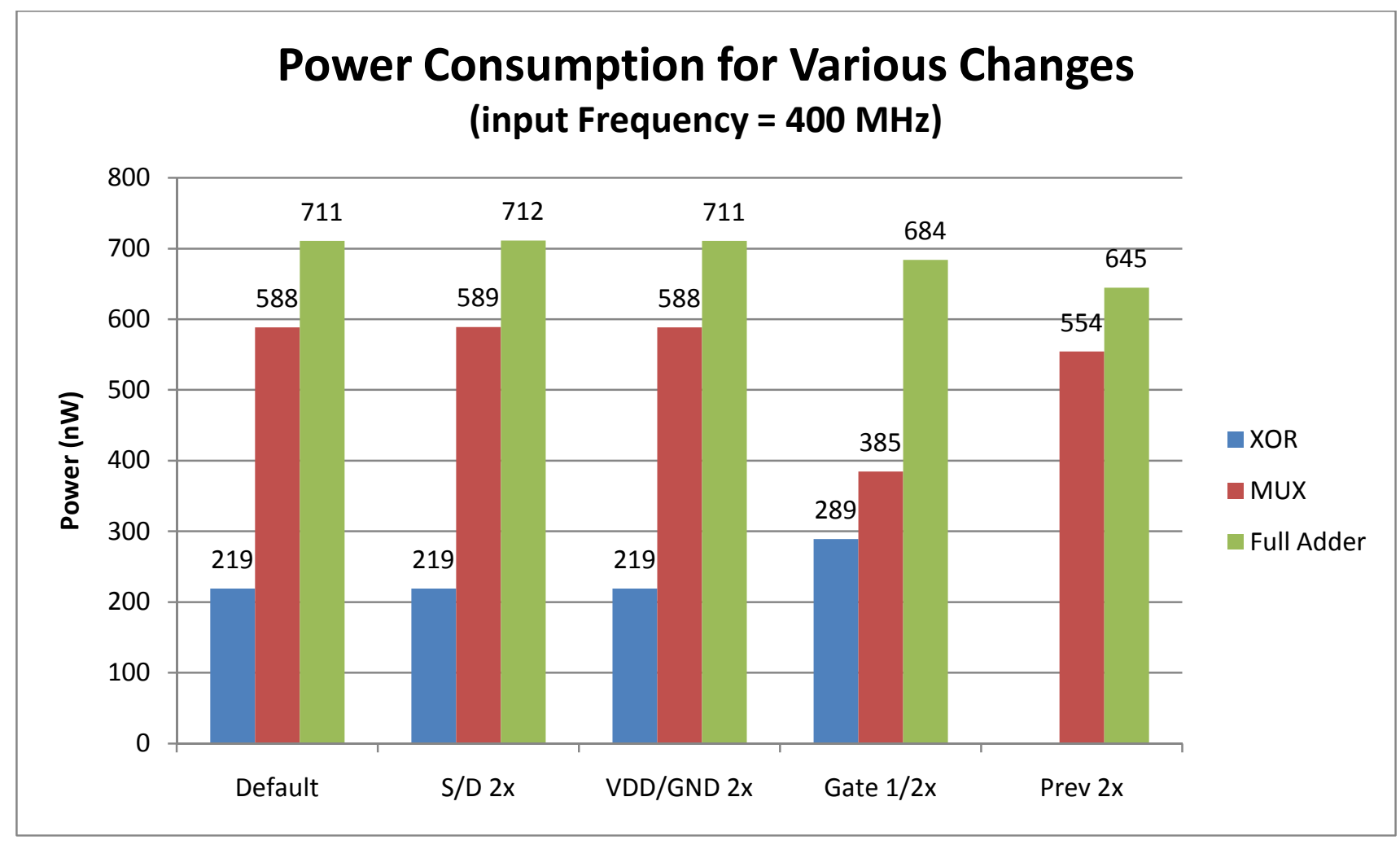

Figure C.4: Power Consumption for Various Changes to Small Circuits at $400 \mathrm{MHz}$

\section{Improving the MUX}

The same changes made to the XOR gate in Chapter 5 were implemented on a 2-input MUX. Again, the weak link was PMOS5, the large PMOS of the output inverter. Doubling the interconnect width at the source/drain locations of the weak link proved to be most effective. This is the easiest way to reduce current density, which is one of the two contributing factors to electromigration. Increasing the width of the power/ground rails had the same effect as before, as the source of the failing FET was VDD. Decreasing the width of the PMOS5 gate also increased MTTF. This reduces the amount of current going to the FET and in turn lowers current density. Finally, changing the speed of the circuit by modifying the stage driving the weak link also slightly increases MTTF. Again, all the changes made to/around the weak link yield another failure site. In larger designs, the changes will hopefully increase the MTTF of the entire design. 


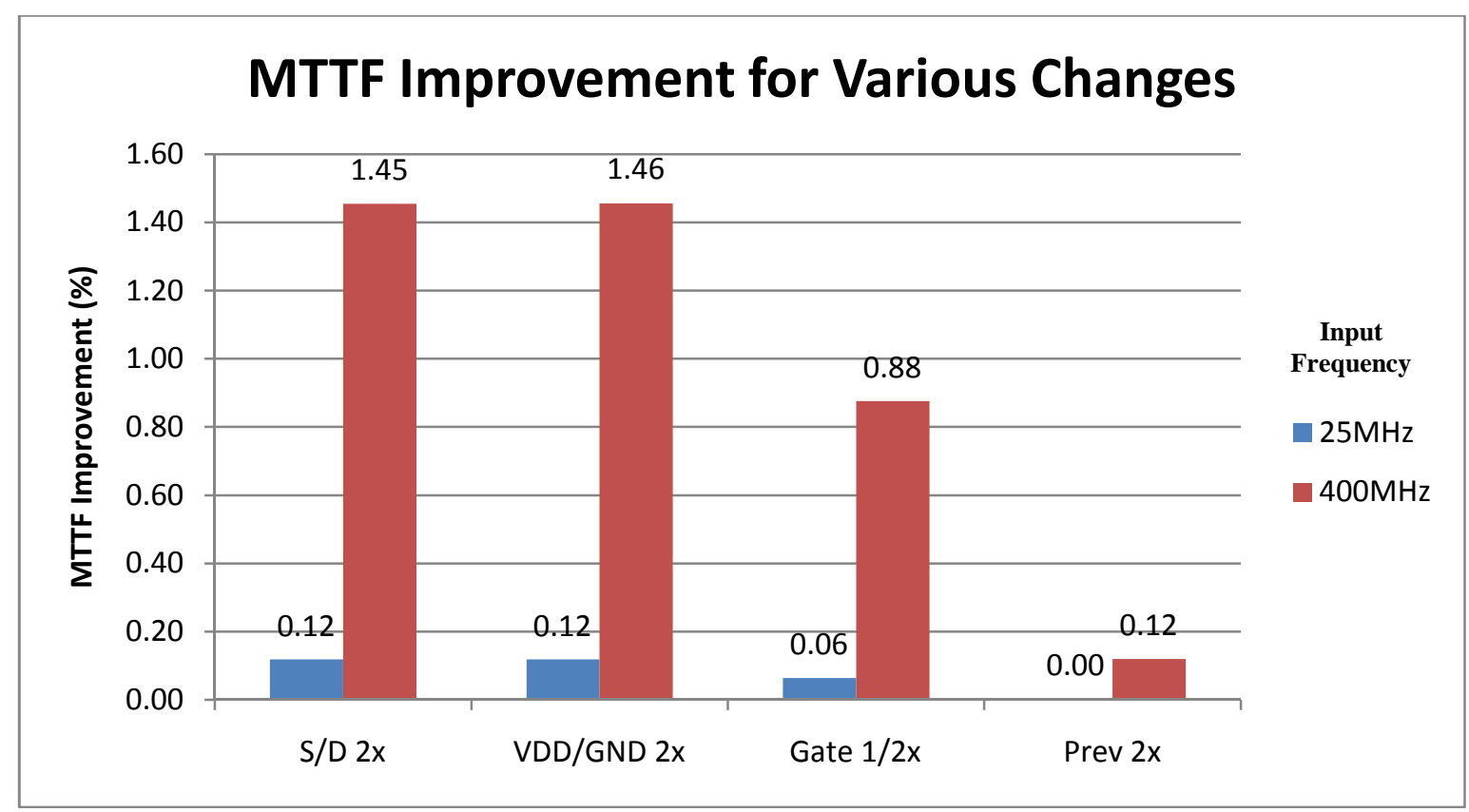

Figure C.5: MTTF Improvement of the MUX for various changes

Figure C.5 shows the changes in MTTF with the various circuit modifications. With the improvement in MTTF come some delay and power tradeoffs. Figures C. 1 through C.4 show the changes on delay and power consumption. As you can see, delay remains the same for changes to interconnect material. However, when the gate of the weak link is reduced in size, the circuit actually speeds up, as it does when the driving stage is increased in size. The results make sense, since it is the output inverter that is being driven by the rest of the circuit. Reducing its size makes the load weaker and allows the circuit to speed up. Also, increasing the size of the driving stage makes that stage stronger and allows it to more effectively drive the output inverter. Power also stays the same for changes in interconnect sizing. Power consumption is reduced for smaller gate size, but variable with changes to the driving stage. It increases slightly at low input frequencies and decreases a bit at higher frequencies. 


\section{Improving a Full Adder}

The changes from the previous sections were again implemented, but this time on a full adder. The weak link was NMOS5 used in the inverter that outputs Cout. Similar performance results were found for the full adder. All changes increased MTTF by marginal amounts with the interconnect sizing at the source/drain of the weak link being the most effective. This time around increasing the size of the power/ground metal lines has no effect on MTTF, as they are not tied to the failure site. Changes in MTTF are much greater for higher input frequencies.

Figure C.6 shows the MTTF improvement for the various changes.

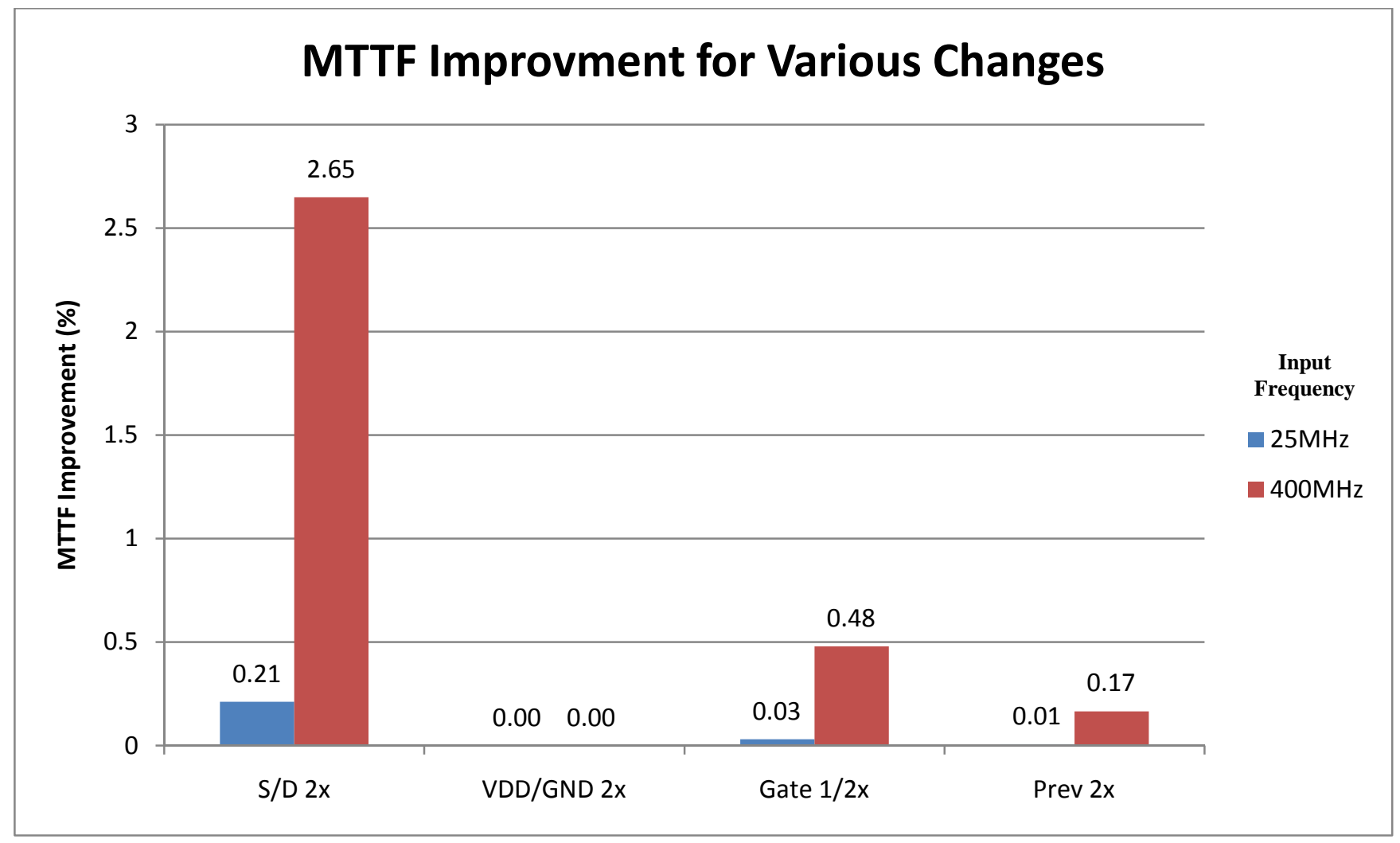

Figure C.6: MTTF Improvement of the Full Adder for various changes

There are some very noticeable changes to delay and power consumption as well. Figures 7.217.24 show these changes respectively. Delay remains the same for all metal changes. This will be 
the case always, as increasing or decreasing the size if the metal lines do not change the amount of current going through them, only the current density. Decreasing the gate size of the weak link makes the inverter weaker, increasing the amount of time the inverter take to actually invert the signal. If I increase the size of the driving stage, this speeds up the circuit and allows the rise/fall times for the inverter to reach their nominal value. Power consumption is reduced in both cases.

\section{Maximum Improvement}

The ideas described in the previous section suggest various ways to improve the lifetime of a circuit. To completely maximize this MTTF, all of the changes can be implemented simultaneously. For the total "fix", the driving stage was increased in size, the weak link decreased in size, and the source/drain metal lines were doubled in width. The figures below show the effects of the entire fix on tested MUX and full adder.

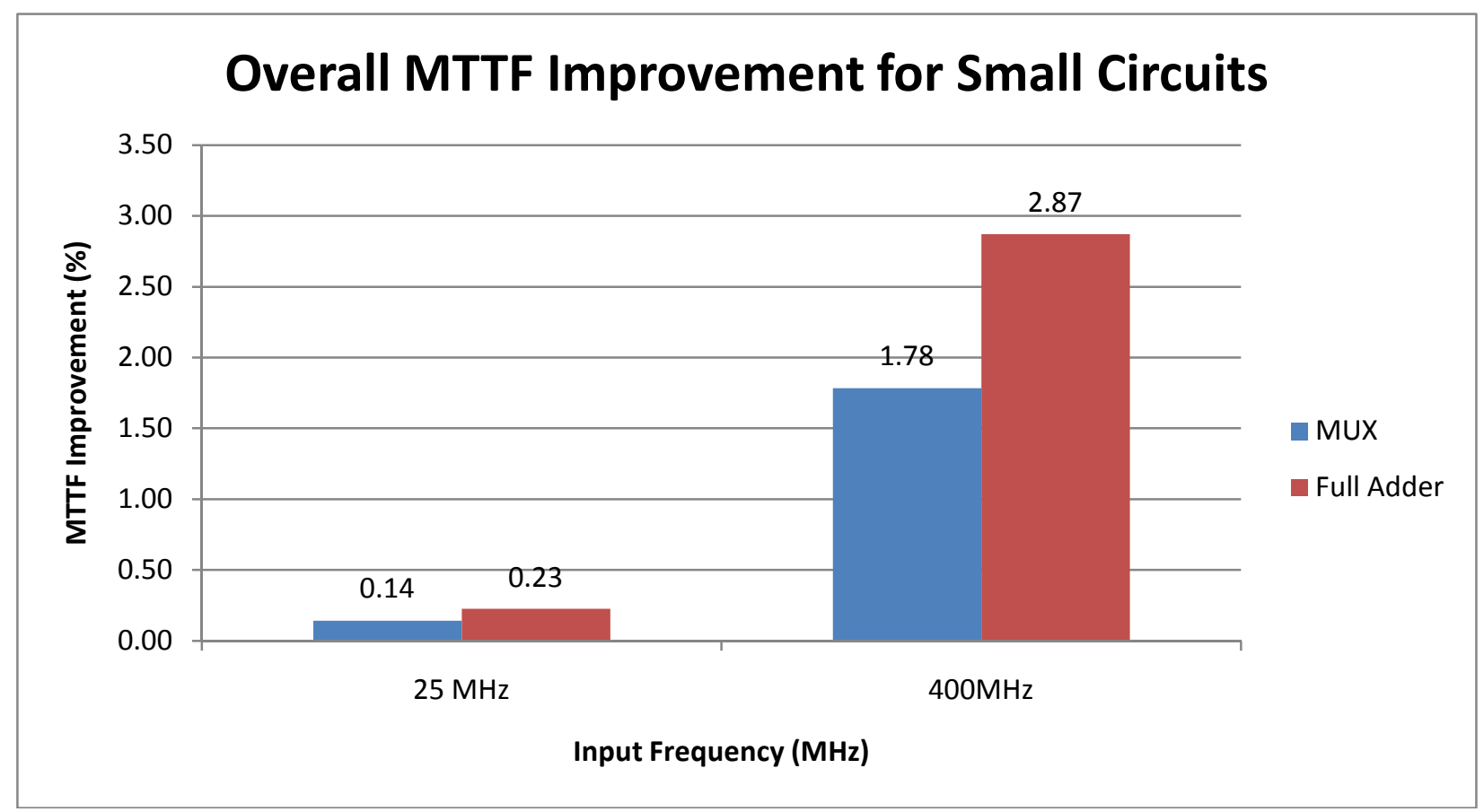

Figure C.7: Overall MTTF Improvement for MUX and Full Adder 


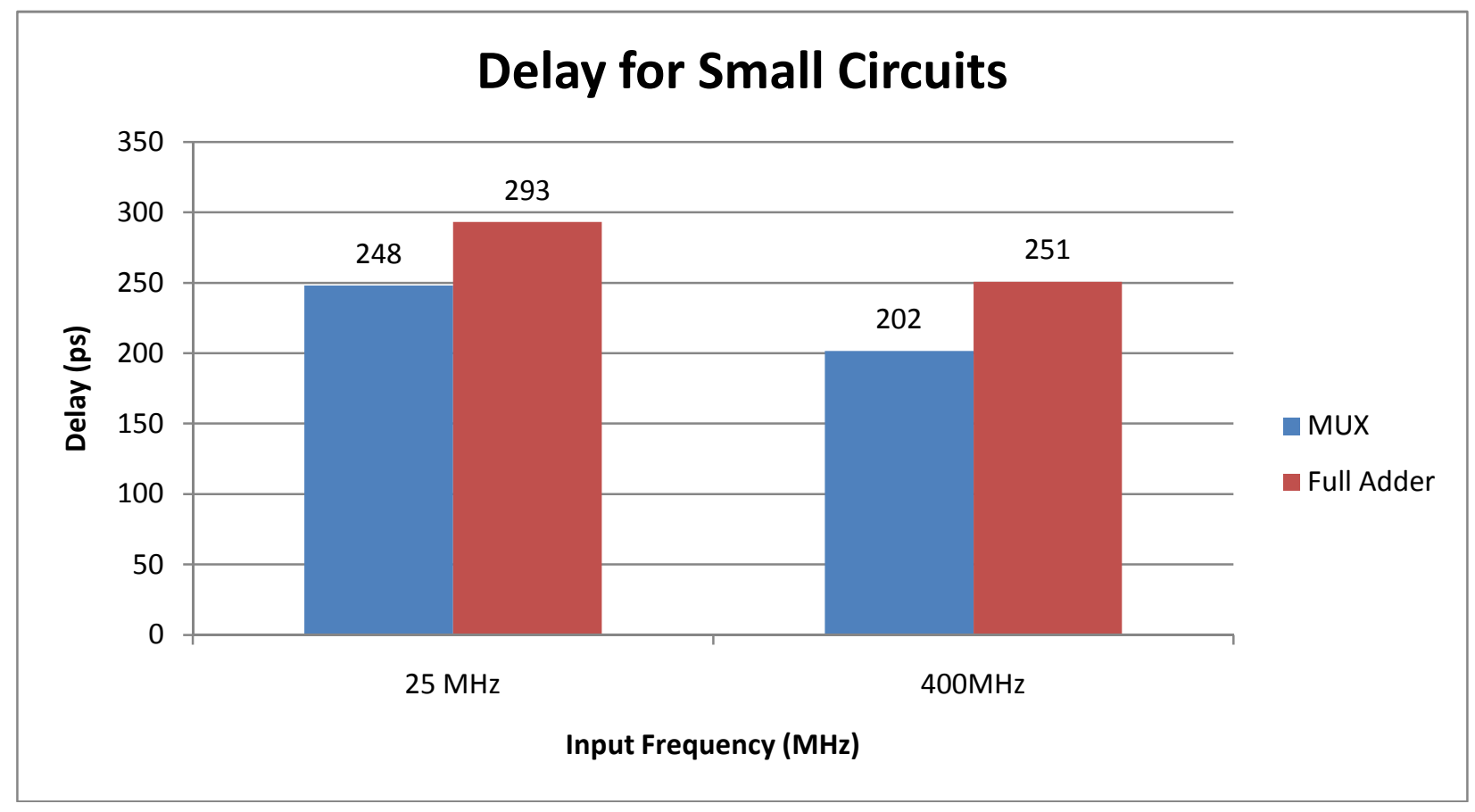

Figure C.8: Delay for the fixed MUX and Full Adder

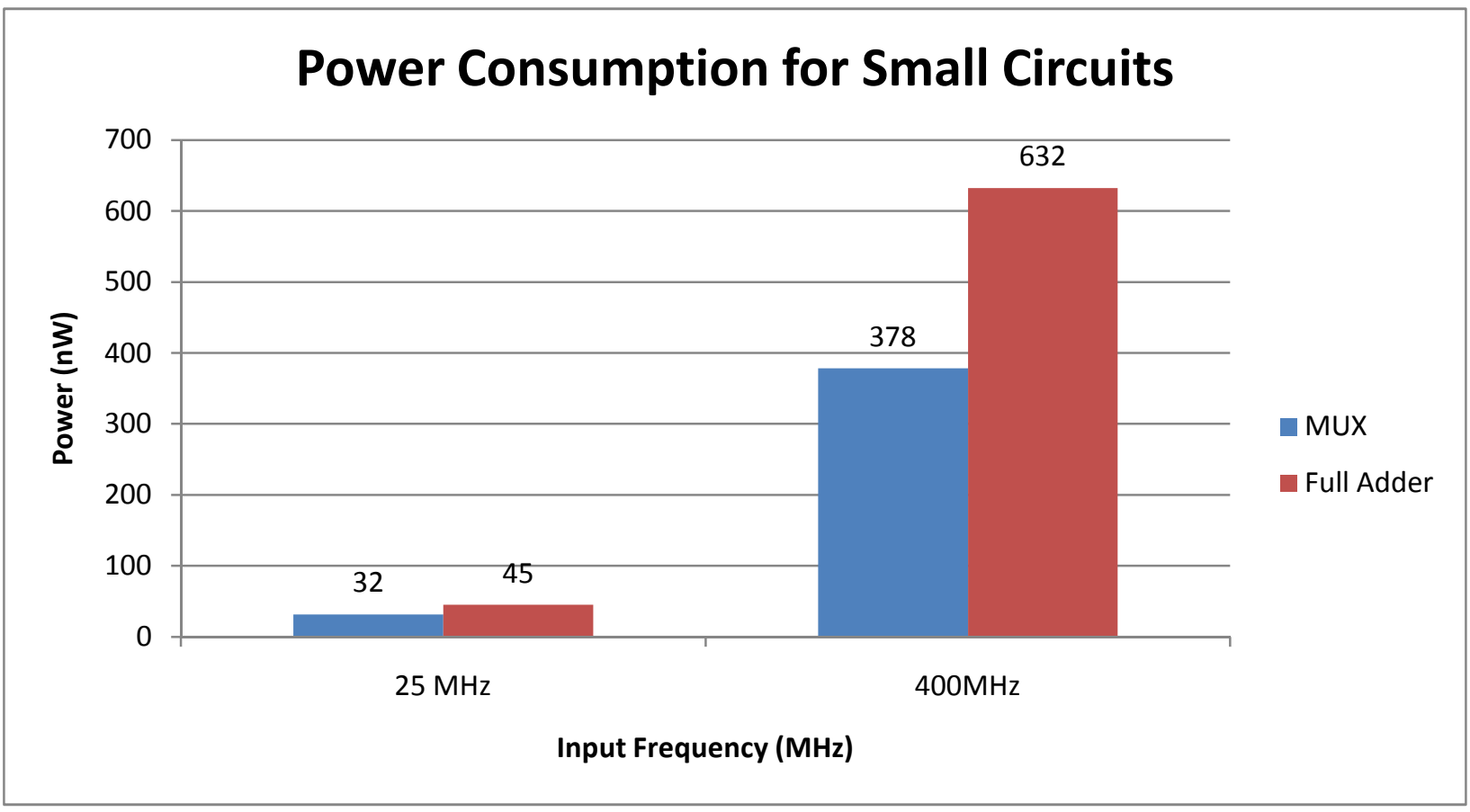

Figure C.9: Power Consumption for fixed MUX and Full Adder 


\section{Summary for Simple Circuits}

It is clear that in order to maximize the lifetime of the circuits, multiple changes had to be made to each design. Each of the changes is beneficial to the circuit in its own way, but a single change is not always best. For example, decreasing the gate size of the weak link increases MTTF, but makes the circuit much slower. Sizing up the driving stage also improves failure time, but sometimes at the cost of power consumption. Ideally, I want to improve MTTF but do so without negatively affecting other performance aspects of the device.

The so-called "fix" for these simple circuits involved three main modifications. Increasing the size of the driving stage helps speed the circuit up minimizing delay. It makes the driving stage stronger and the load that much easier to drive. Decreasing the size of the weak link consumes much less power and minimizes the amount of current going through that FET. Finally, doubling the width of the metal lines surrounding the weak link halves the current density associated with the metal. Increasing the driving stage does speed up the circuit, but consumes more power. However, this extra power consumption and reduction in delay is countered with the decrease in gate size of the weak link. That decrease uses less power but at the cost of delay. The increased metal sizing is the most effective way of reducing current density but requires more area, which with today's scaling down process could pose a problem. As can be seen in the figures from Section 5.4, the "fix" provides a strong increase on MTTF, especially at faster input frequencies. Overall, the delay remains about the same and power consumption is significantly reduced. 


\section{Appendix D: List of Acronyms}

\begin{tabular}{|l|l|}
\hline $\mathrm{Al}_{2} \mathrm{O}_{3}$ & Aluminum Oxide \\
\hline $\mathrm{DC}$ & Direct Current \\
\hline $\mathrm{DRC}$ & Design Rule Checker \\
\hline EDA & Electronic Design Automation \\
\hline EDIF & Electronic Design Interchange Format \\
\hline TDDB & Time Dependant Dielectric Breakdown \\
\hline EM & Electromigration \\
\hline PDN & Pull Down Network \\
\hline FET, MOS, MOSFET & Metal-Oxide Semiconductor Field-Effect \\
\hline IC & Integristor \\
\hline KS & Kogge-Stone \\
\hline LEF/DEF & $\begin{array}{l}\text { Library Exchange Format/Design Exchange } \\
\text { Format }\end{array}$ \\
\hline MOSIS & $\begin{array}{l}\text { Metal Oxide Semiconductor Implementation } \\
\text { Service }\end{array}$ \\
\hline MTTF & Mean Time to Failure \\
\hline MUX & Multiplexor \\
\hline NMOS, N-MOSFET & n-Channel MOSFET \\
\hline OPL & Output Prediction Logic \\
\hline PAL & Programmable Array Logic \\
\hline PCB & Printed Circuit Board \\
\hline PF & Poole-Frenkel \\
\hline PMOS, P-MOSFET & p-Channel MOSFET \\
\hline PPA & Parallel Prefix Adder \\
\hline RC & Resistor-Capacitor Circuit \\
\hline RCA & Ripple Carrier Adder \\
\hline SRAM & Static RAM (Random Access Memory) \\
\hline EM & Electromigration \\
\hline VHDL & $\begin{array}{l}\text { VHSIC (Very High Speed Integrated Circuits) } \\
\text { Hardware Description Language }\end{array}$ \\
\hline VLSI & Very Large Scale Integration \\
\hline & \\
\hline
\end{tabular}

Table D.1: List of Acronyms 


\title{
Appendix E: List of Equations
}

\author{
$M T T F=A w j^{-n} e^{\left(\frac{Q}{k T}\right)}$ \\ Equation 2.1 \\ $M T T F=200 * e^{(3.867 E-08 * J)}+216.39 * e^{(-1.814 E-06 * J)}-T$ \\ Equation 2.2 \\ $M T T F=200 * e^{(3.867 E-08 * J)}+216.39 * e^{(-1.814 E-06 * J)}-100$ \\ Equation 2.3 \\ $M T T F=200 * e^{(3.867 E-08 * J)}+216.39 * e^{(-1.814 E-06 * J)}-150$ \\ Equation 2.4 \\ $M T T F=200 * e^{(3.867 E-08 * J)}+216.39 * e^{(-1.814 E-06 * J)}-200$ \\ Equation 2.5 \\ $M T T F=200 * e^{(3.867 E-08 * J)}+216.39 * e^{(-1.814 E-06 * J)}-250$ \\ Equation 2.6
}

For $T=100: M T T F=308.3401 e^{-0.000001089 J}$

Equation 2.7

For $T=150: M T T F=260.6282 e^{-0.000001385 J}$

Equation 2.8

For $T=200: M T T F=215.0316 e^{-0.000001906 J}$

Equation 2.9

For $T=250: M T T F=177.1201 e^{-0.000002996 J}$

Equation 2.10

$\left(308.3401+N\left(100-T_{S}\right)\right) e^{\left(-1.089 E-06+M\left(100-T_{S}\right)\right) J}$

Equation 2.11 
$(308.3401+N(100-150)) e^{(-1.089 E-06+M(100-150)) J}=260.6282 e^{-0.000001385 J}$

Equation 2.12

$(308.3401+N(100-200)) e^{(-1.089 E-06+M(100-200)) J}=215.0316 e^{-0.000001906 J}$

Equation 2.13

$(308.3401+N(100-250)) e^{(-1.089 E-06+M(100-250)) J}=177.1201 e^{-0.000002996 J}$

Equation 2.14

$$
J=\frac{A}{w * h}
$$

Equation 2.15

$$
P=\frac{1}{2} f_{C} C_{L} V_{D D}^{2}
$$

Equation 4.1 